\title{
POTENTIAL EFFECT ON NATURAL GAS WELLS ON ALLUVIAL GROUNDWATER CONTAMINATION AT THE KANSAS CITY PLANT
}

\author{
D. A. Pickering \\ A. D. Laase \\ D. A. Locke*
}

*Oak Ridge Institute for Science and Education

Published: May 1993

Prepared by the

OAK RIDGE NATIONAL LABORATORY

Oak Ridge, Tennessee 37831

managed by

MARTIN MARIETTA ENERGY SYSTEMS, INC.

for the

U.S. DEPARTMENT OF ENERGY

under contract No. DE-ACO5-84OR21400 


\section{CONTENTS}

FIGURES $\ldots \ldots \ldots \ldots \ldots \ldots \ldots \ldots \ldots \ldots \ldots \ldots$ iv

EXECUTTVE SUMMARY $\ldots \ldots \ldots \ldots \ldots \ldots \ldots \ldots \ldots \ldots \ldots$ ix

1. INTRODUCTION $\ldots \ldots \ldots \ldots \ldots \ldots \ldots \ldots \ldots \ldots \ldots \ldots \ldots \ldots \ldots \ldots \ldots \ldots$

1.1 PURPOSE AND SCOPE $\ldots \ldots \ldots \ldots \ldots \ldots \ldots \ldots \ldots$

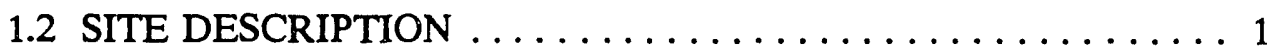

2. ENVIRONMENTAL SETTING $\ldots \ldots \ldots \ldots \ldots \ldots \ldots \ldots \ldots$

2.1 GEOLOGY ..................... 5

2.2 HYDROGEOLOGY ................... 9

2.2.1 Hydrologic Units ................. 9

2.2.2 Area and Amounts of Recharge ........... 9

2.2.3 Groundwater Flow Directions ............ 10

2.2.4 Groundwater Flow Velocities ............ 10

2.3 CONTAMINANTS OF CONCERN ............. 12

3. OIL AND GAS EXPLORATION AND PRODUCTION $\ldots \ldots \ldots \ldots .17$

3.1 HISTORY AND METHODS OF THE PRE-WORLD

WAR II ERA $\ldots \ldots \ldots \ldots \ldots \ldots \ldots \ldots \ldots \ldots \ldots \ldots$

3.1 .1 Regional History $\ldots \ldots \ldots \ldots \ldots \ldots \ldots \ldots \ldots$

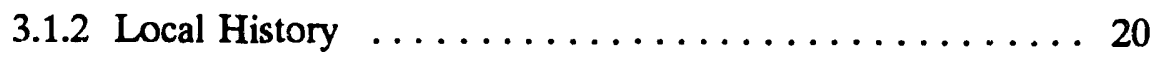

3.1.3 Drilling and Abandonment Methods ......... 23

$3.2 \mathrm{KCP}$ NATURAL GAS WELLS $\ldots \ldots \ldots \ldots \ldots \ldots \ldots \ldots \ldots$

3.2.1 Clair Report, $1943 \ldots \ldots \ldots \ldots \ldots \ldots \ldots . \ldots \ldots$

3.2.2 Information from MDNR, $1992 \ldots \ldots \ldots \ldots \ldots \ldots$

3.2.3 Information from KCP Employee $\ldots \ldots \ldots \ldots \ldots$ 


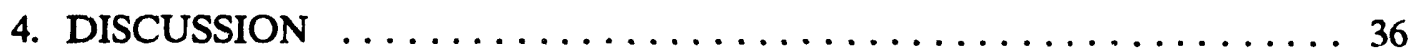

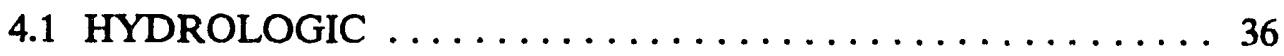

4.2 STRATIGRAPHIC $\ldots \ldots \ldots \ldots \ldots \ldots \ldots \ldots \ldots \ldots \ldots \ldots \ldots$

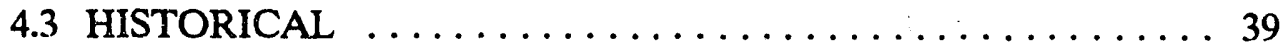

4.4 RECOMMENDATIONS $\ldots \ldots \ldots \ldots \ldots \ldots \ldots \ldots \ldots . \ldots . \ldots . \ldots$

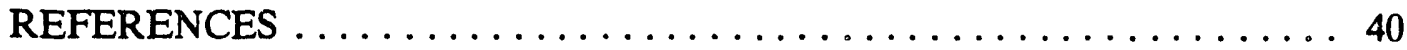

ACRONYMS AND INITIALISMS $\ldots \ldots \ldots \ldots \ldots \ldots \ldots \ldots \ldots, 43$

APPENDIX A Lithologic log of a well in Sect. 36, T48N, R33W, Jackson

County, Missouri

APPENDIX B Well Water-Level Elevations

APPENDIX C Analytical Data from Selected Weils 


\section{FIGURES}

1. I ration of $\mathrm{KCP}$ in the greater Kansas City area ............ 2

2. Estimated location of old natural gas wells at $\mathrm{KCP}$, based on old map found at $\mathrm{KCP} \ldots \ldots \ldots \ldots \ldots \ldots \ldots \ldots \ldots \ldots \ldots$

3. Contour map of bedrock surface beneath KCP ............6

4. Stratigraphic section of the Kansas City and Pleasanton Groups of Pennsylvanian using modern formation nomenclature $\ldots \ldots \ldots \ldots$

5. Cross section of the Pleasanton and Kansas City Groups at KCP . . . . 8

6. Potentiometric map of the lower zone in the alluvial aquifer ........ 11

7. Depiction of the TCE plume in the TCE Still Area ............ 14

8. Depiction of the DCE plume in the TCE Still Area ............ 15

9. Depiction of the chloroethene plume in the TCE Still Area ......... 16

10. Location of KCP within greater Kansas City, Jackson County, neighboring counties, and regional townships $\ldots \ldots \ldots \ldots \ldots \ldots \ldots \ldots \ldots \ldots \ldots \ldots \ldots$

11. Oil and gas fields in Johnson County, Karis. in $1954 \ldots \ldots \ldots \ldots$

12. Location of historical towns close to $\mathrm{KCP} \ldots \ldots \ldots \ldots \ldots \ldots \ldots$

13. Location of the seven wells shown on a Clair report map (1943), transposed on a modern map of KCP ................ 26

14. Location of old wells shown on map "Missouri Oil and Gas Wells of Record, 1860 to Present (1987)", transposed onto modern map of KCP . . . . . . 27 


\section{EXECUTIVE SUMMARY}

This report is the result of a request for further information about several abandoned natural gas wells at the U.S. Department of Energy's Kansas City Plant (KCP). The request was prompted by an old map showing several, possibly eight, natural gas wells located under or near what is now the southeast corner of the Main Manufacturing Building at KCP. Volatile organic compound contamination in the alluvial aquifer surrounding the gas wells might possibly contaminate the bedrock aquifer if the gas wells still exist as conduits.

Several circumstances exist that make it doubtful that contamination is entering the bedrock aquifers: 1) because regional groundwater flow in the bedrock beneath the KCP is expected to be vertically upward, contaminants found in the alluvial aquifer should not migrate down the old wells; 2) because of the low hydraulic conductivity of the bedrock units, contaminant transport would be extremely slow if the contaminants were migrating down the wells; and 3) casing, apparently set through the alluvium in all of the wells, would have deteriorated and may have collapsed; if the casing collapsed, the silty clays in the alluvium would also collapse and seal the well.

No definitive information has been discovered about the exact location of the wells. No further search for or consideration of the old gas wells is recommended. 


\section{INTRODUCTION}

\subsection{PURPOSE AND SCOPE}

This report is the result of a request for further information about several abandoned natural gas wells at the U.S. Department of Energy's (DOE) Kansas City Plant (KCP) (Fig. 1). The request was prompted by an old map showing several, possibly eight, natural gas wells located under or near what is now the southeast corner of the Main Manufacturing Building at KCP (Fig. 2). There is concern about possible transmission of contamination from the alluvial groundwater to deeper aquifers, with the old gas wells acting as conduits.

The investigation began with a search for the history of oil and gas exploration in the Kansas City area prior to construction of the plant in 1942. The search yielded information regarding: the identity of geologic formations that produced oil and gas in the area, the depth of the wells, their proximity to KCP, and the methods used for drilling and abandonment. Detailed information from the time period, especially about specific wells, was generally not documented. In order to address the likelihood of contaminant transport through the old wells, the investigation also included a review of pertinent data (the contaminants in the area, stratigraphy, hydrogeology) and interpretation of these data.

\subsection{SITE DESCRIPTION}

$\mathrm{KCP}$ is a government-owned manufacturing plant operated by Allied-Signal, Inc., on behalf of the DOE Kansas City Area Office. The site is located in Jackson County, Mo., in south Kansas City, northeast of the intersection of Bannister Road and Troost Avenue. It is bordered by Indian Creek to the south and the Blue River to the east. The facility occupies 113 acres within a 300 acre 


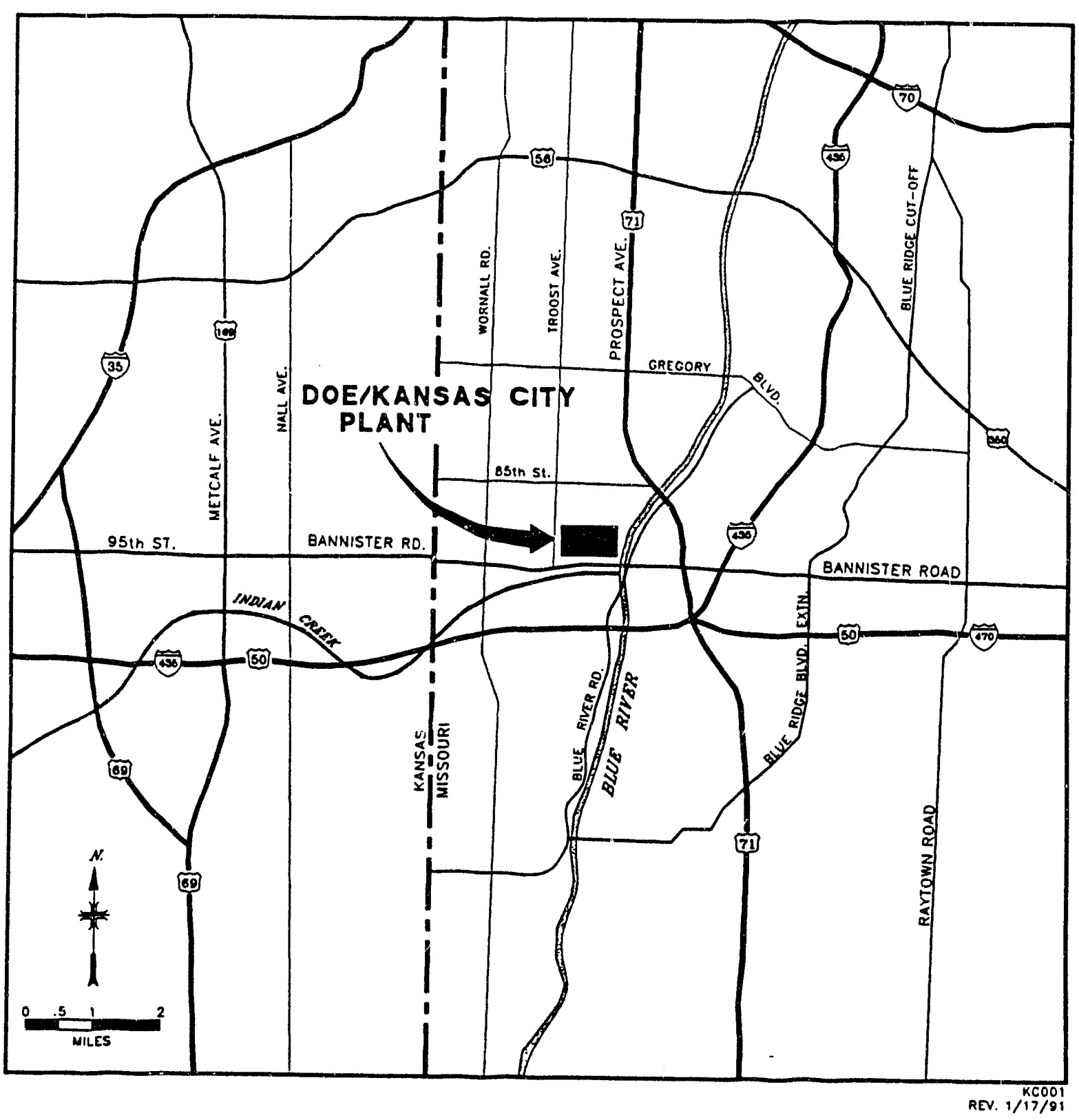

Fig. 1. Location of KCP in the greater Kansas City area. 


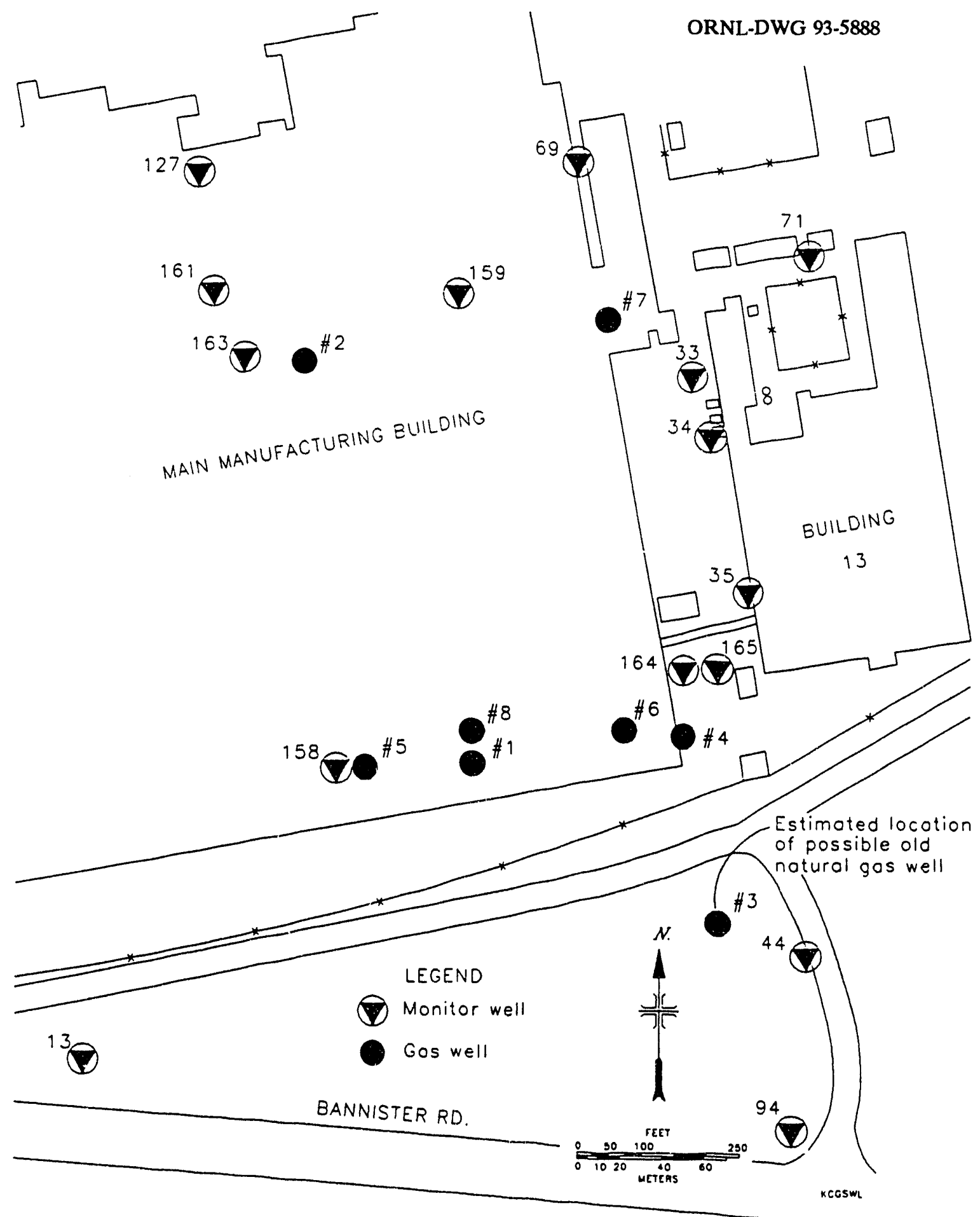

Fig. 2. Estimated location of old natural gas wells at KCP, based on old map found at KCP. 
federal governmeni complex. The facility, whose prime occupant is Allied-Signal, Inc., Kansas City Division, also houses the General Services Administration, the Marine Corps, and several other federal agencies. The Internal Revenue Service has a facility located directly east of the DOE facility.

The main building at the DOE/KCP was built to manufacture aircraft engines. The facility was built by the U.S. Navy for the Pratt \& "Whitney Corporation (KCD 1987). Building construction was initiated in mid-1942; engine manufacturing began in late 1943. Prior to 1942 , the area was dedicated to agriculture, except for a brief period when it was occupied by an automobile racetrack.

Plant operations conducted in the early days of facility occupancy have resulted in groundwater contamination, which is being investigated and remediated under the auspices of the DOE's Environmental Restoration Program.

The old gas wells are closest to the TCE Still Area investigation site, one of several sites being investigated (U.S.DOE 1991c, 1990d). Several of the maps and figures used in this report are the result of investigations conducted in support of the TCE Still Area characterization.

\section{ENVIRONMENTAL SETTING}

Numerous documents describe the environmental setting of KCP. Korte et al. (1985) and Fleischhauer et al. (1986) described the initial phases of the site characterization. More recent information may be found in groundwater assessment plans for the contaminated sites (U.S.DOE 1990b, 1990c, 1990d, and 1990e) and in recent annual reports (U.S.DOE 1990a, 1991a).

\section{GEOLOGY}

Kansas City is located in the middle of a 150-mile-wide outcropping of Pennsylvanian rocks that extend in a north-south direction through western Missouri 
and eastern Kansas. The area slopes gently toward the Forest City Basin in northwest Missouri. The average change in elevation in this area is 10 to $12 \mathrm{ft} / \mathrm{mile}$.

Strata underlying the KCP consist of approximately $45 \mathrm{ft}$ of unconsolidated Quaternary alluvium resting on some $2400 \mathrm{ft}$ of Paleozoic strata. A Precambrian crystalline basement complex underlies the paleozoic section. Upper Pennsylvanian rocks of the Kansas City Group are exposed as bluffs along the valley margins. Figure 3 is a contour map of the top of the bedrock, showing the bluffs north of the plant and the gradual slope under the valley fill. A stratigraphic section showing the Pleasanton Group is shown in Fig. 4.

Bedrock beneath the alluvium is the Knobtown sandstone, followed by interbedded silty shale layers of the Pleasanton Group, the Hepler sandstone, more silty shale of the Pleasanton, and then the Marmaton Group at about $140 \mathrm{ft}$ (from log of well KC84-23). Well KC84-23 was a bedrock well drilled to a depth of $140.7 \mathrm{ft}$ in 1984, $1500 \mathrm{ft}$ east of the southeast corner of the Main Manufacturing Building (Korte et al. 1985).

Both the Knobtown sandstone and shales of the Pennsylvanian Pleasanton Group (Fig. 5) directly contact the Blue River alluvium under KCP. The Knobtown is a marine sandstone ranging in thickness from approximately 5 to $10 \mathrm{ft}$. The Knobtown is present in the upper $30 \mathrm{ft}$ of the Pl'isanton Group except where it has been removed by Quaternary erosion (U.S.DOE 1984). The Hepler sandstone is present in the lower Pleasanton and also underlies KCP. The top of the Hepler sandstone is approximately $40 \mathrm{ft}$ below the base of the alluvium and is hydraulically isolated from the overlying alluvium by impermeable shales of the Pleasanton Group.

The Marmaton Group of the middle Pennsylvanian consists of interbedded shales and limestones and is approximately $300 \mathrm{ft}$ thick in eastern Kansas and western Missouri. Below the Marmaton lies the Cabaniss and Krebs Formations of the Cherokee Group. Each of these formations ranges from 100 to $180 \mathrm{ft}$ thick (Heckel 1978). 
ORNL-DWG 93-5889

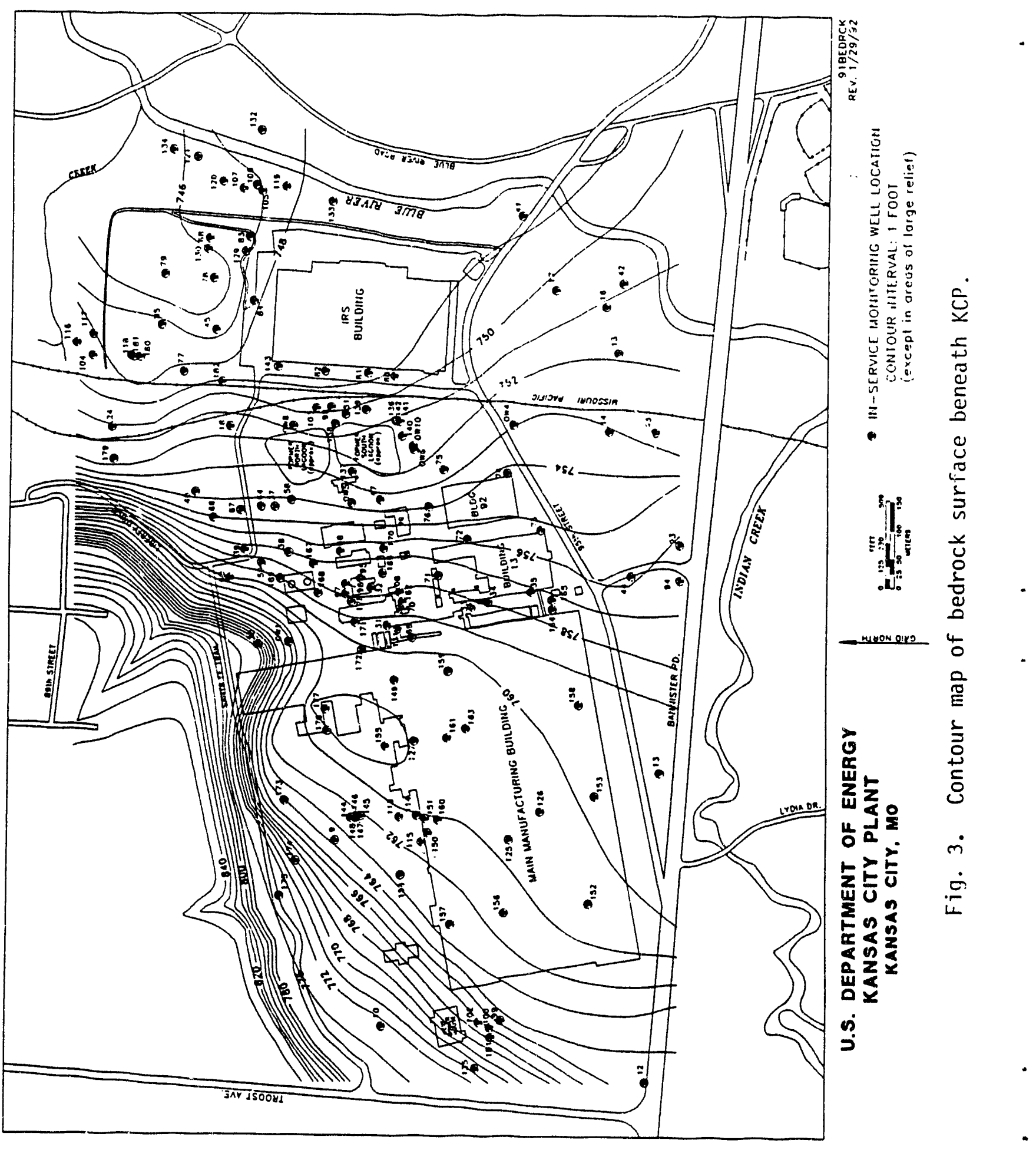




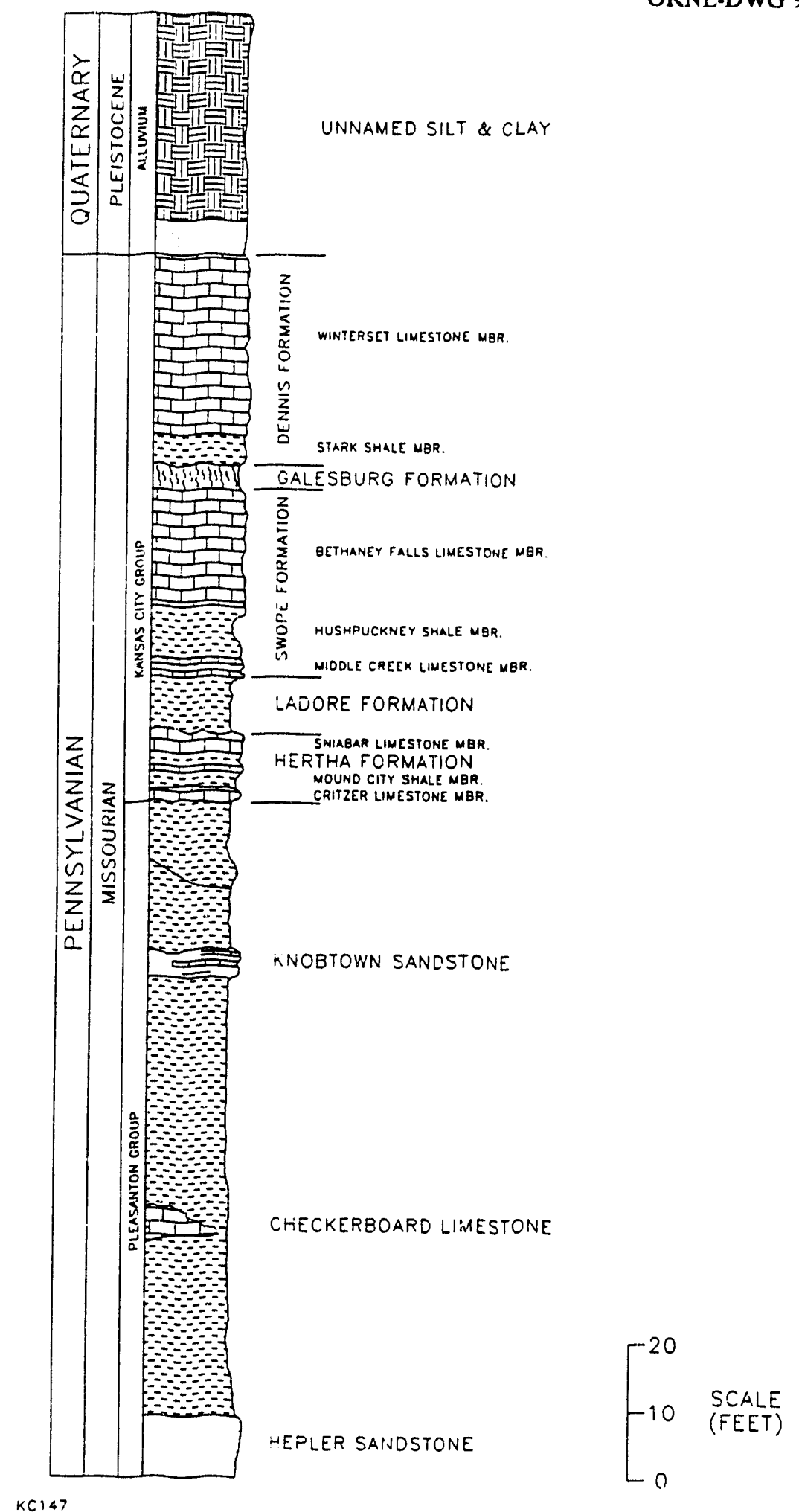

ORNL-DWG $93-5890$

Fig. 4. Stratigraphic section of the Kansas City and Pleasanton Groups of Pennsylvanian using modern formation nomenclature. 
8
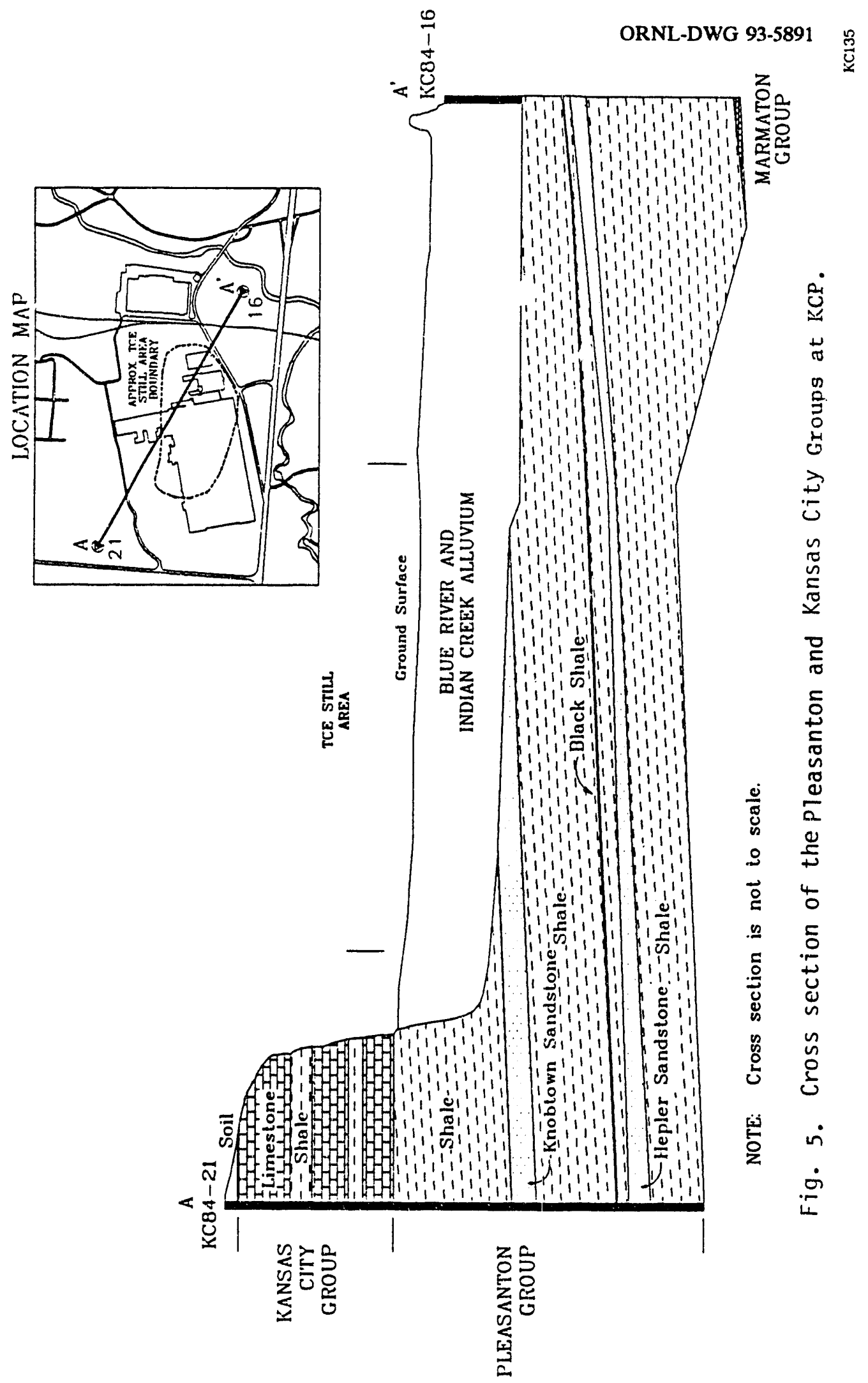


\section{HYDROGEOLOGY}

\subsection{Hydrologic Units}

The $45 \mathrm{ft}$ of unconsolidated alluvium is generally divided into two units (upper and lower) for hydrologic purposes. The units are both silty clays exhibiting very low flow characteristics (Sect. 2.2.4) and are separated by a low permeability green clay.

The bedrock units beneath the alluvium are the Knobtown sandstone, shales, and the Hepler sandstone of the Pleasanton Group. Hydrologic properties of these units are described in Sect. 2.2.4. Water bearing properties of the Pleasanton Group are reported as low, with yields of 1 to $3 \mathrm{gal} / \mathrm{min}$. Dissolved solids are mostly high (>1000 ppm) at all depths in the group (Gann et al. 1974).

Beneath the Hepler sandstones lie the Marmaton and Cherokee Groups of the Pennsylvanian. These groups have been characterized as low-yielding units for water ( 1 to $3 \mathrm{gal} / \mathrm{min}$ ), yields adequate for domestic purposes only. Groundwater in the units is mineralized, and groundwater quality in Jackson County does not meet acceptable standards for drinking. Total dissolved solids range from 10,000 to 40,000 ppm; less than 1000 ppm is considered fresh water (Gann et al. 1974).

\section{Area and Amounts of Recharge}

$\mathrm{KCP}$ itself is mostly covered with pavement; therefore, little on-site recharge occurs. Several years of monitoring have demonstrated that water levels across KCP typically show minor variations, usually within two feet (U.S.DOE 1991a). The surrounding streams are the major discharge area for the alluvial aquifer (U.S.DOE 1992a). The amount of groundwater recharge from the alluvium to the underlying Paleozoic rocks is negligible in comparison to the total flow through the alluvium (U.S.DOE 1992a). 


\section{Groundwater Flow Directions}

The potentiometric surface of the upper and lower more-permeable units of the alluvium beneath KCP indicate that regional groundwater flow within these units is to the south and east (radial) and that the Blue River and Indian Creek are gaining streams. Groundwater samples collected from bedrock welis installed down-dip from contaminant sources have remained clean (U.S.DOE 1991a). Figure 6 shows a potentiometric map of the lower completions of the alluvial monitoring wells at the plant.

\subsection{Groundwater Flow Velocities}

\section{Alluvial}

Four pumping tests have been performed at KCP: the green-clay aquifer test (U.S.DOE 1992a), the Northeast Area test (Madril et al. 1986), the Tank Farm Area test (U.S.DOE 1990c), and the South Lagoon test (U.S.DOE 1991b). Hydraulic conductivities and storage coefficients calculated from three of the aquifer tests (Northeast Area, Tank Farm Area, and South Lagoon Area) ranged from 0.6 to $2.3 \mathrm{ft} / \mathrm{d}$ and $5 \times 10^{-4}$ to $2 \times 10^{-3}$ respectively. The green-clay aquifer test was conducted to determine the degree to which a middle green-clay zone restricts the vertical movement of groundwater between the upper and lower alluvial units at KCP. Groundwater flow velocities calculated from the values reported above range from $0.023 \mathrm{ft} /$ day to $0.053 \mathrm{ft} /$ day. These relatively low flow velocities arc reasonable for the fine-grain material comprising the alluvial aquifer at KCP.

\section{Bedrock}

Results from packer tests conducted on bedrock coreholes during previous investigations indicate hydraulic conductivities in bedrock below the measurable 
ORNL-DWG 93-5892

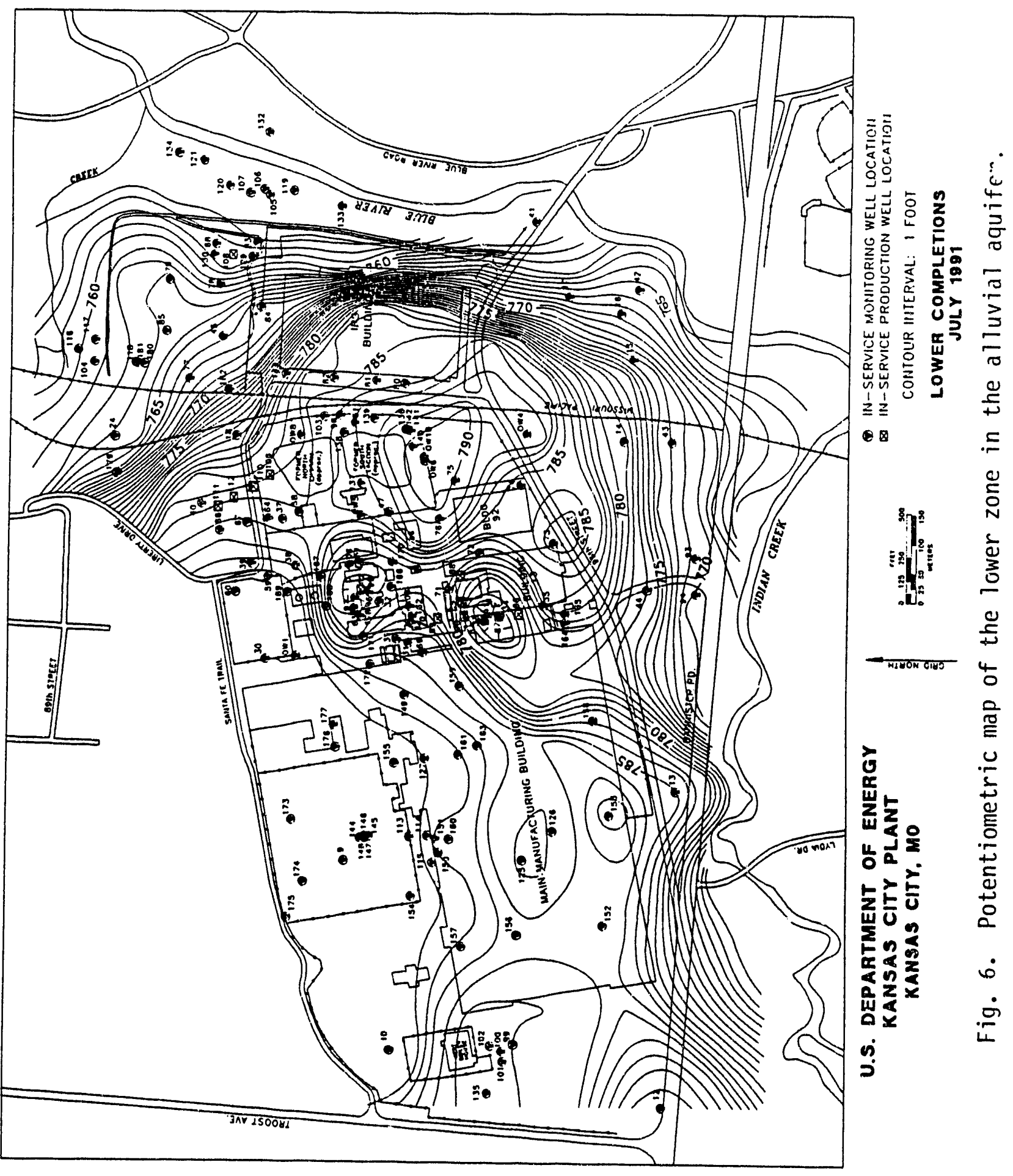


range. Because packer tests can measure hydraulic conductivities in the range of $0.0001 \mathrm{ft} / \mathrm{day}$, it is a safe assumption that the hydraulic conductivity of the Pleasanton shale is in the $0.00001 \mathrm{ft} /$ day or lower range (U.S.DOE 1992b). Additionally, there is no indication of fractures in the shale. Consequently, the Pleasanton shale is an effective barrier to the vertical migration of groundwater.

The hydraulic conductivity of the Knobtown sandstone ranges from 0.005 to $0.04 \mathrm{ft} /$ day. Because the unit is in hydraulic communication with the overlying alluvium, groundwater flow rates are of concern. Using the same range of hydraulic gradients as in the overlying alluvium (0.02 to 0.002) and an assumed effective porosity of 0.12 , groundwater migrates at a rate of 0.24 to $2.4 \mathrm{ft} / \mathrm{yr}$ in the Knobtown sandstone (U.S.DOE 1990b).

As with the Pleasanton shales, the hydraulic conductivity in the Hepler sandstone was below the range at which packer tests can yield a reliable measurement. The Hepler sandstone exhibited no-flow conditions in well KC84-22 and $0.024 \mathrm{ft} /$ day in well $\mathrm{KC} 84-23$. This evidence indicates that the Hepler is a lowpermeable formation.

The hydraulic conductivity of the Hepler sandstone, combined with the fact that the Hepler sandstone is isolated from the alluvium by $35 \mathrm{ft}$ of impermeable Pleasanton shale, suggests that contamination of this unit is not a concern. If a preferred pathway has been created by a gas well, the Hepler sandstone is not a conductive formation.

\section{CONTAMINANTS OF CONCERN}

The contaminant plume of concern is the solvent plume associated with the TCE Still Area. The three prominent contaminants in the plume are: trichloroethene (TCE), 1,2-dichloroethene (total) (1,2-DCE), and chloroethene. Figures 7, 8 , and 9 are graphical defictions of the contaminant plume for each of the three 
ORNL-DWG 93-5893

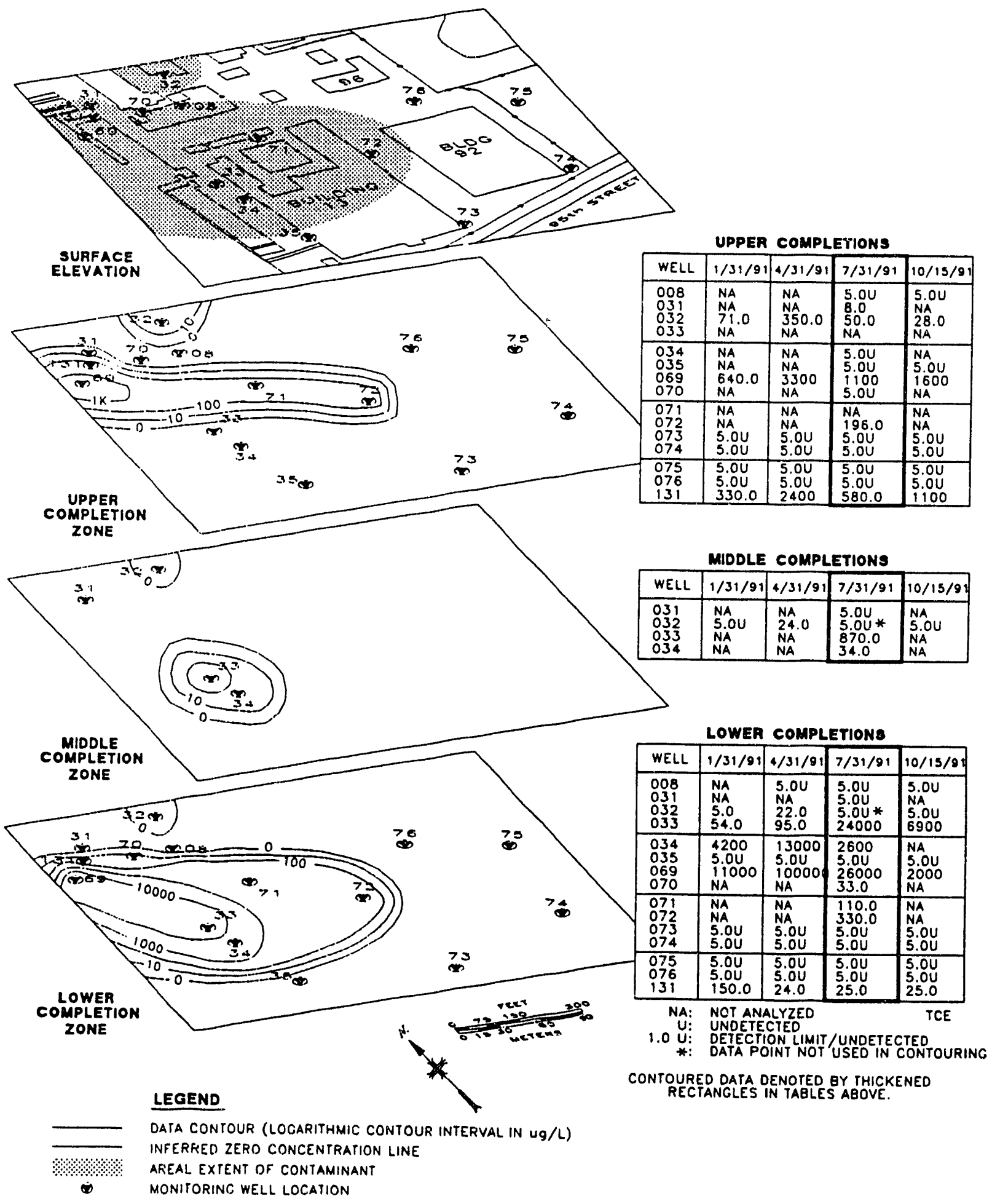

Fig. 7. Depiction of the TCE plume in the TCE Still Area. 
ORNL-DWG 93-5894

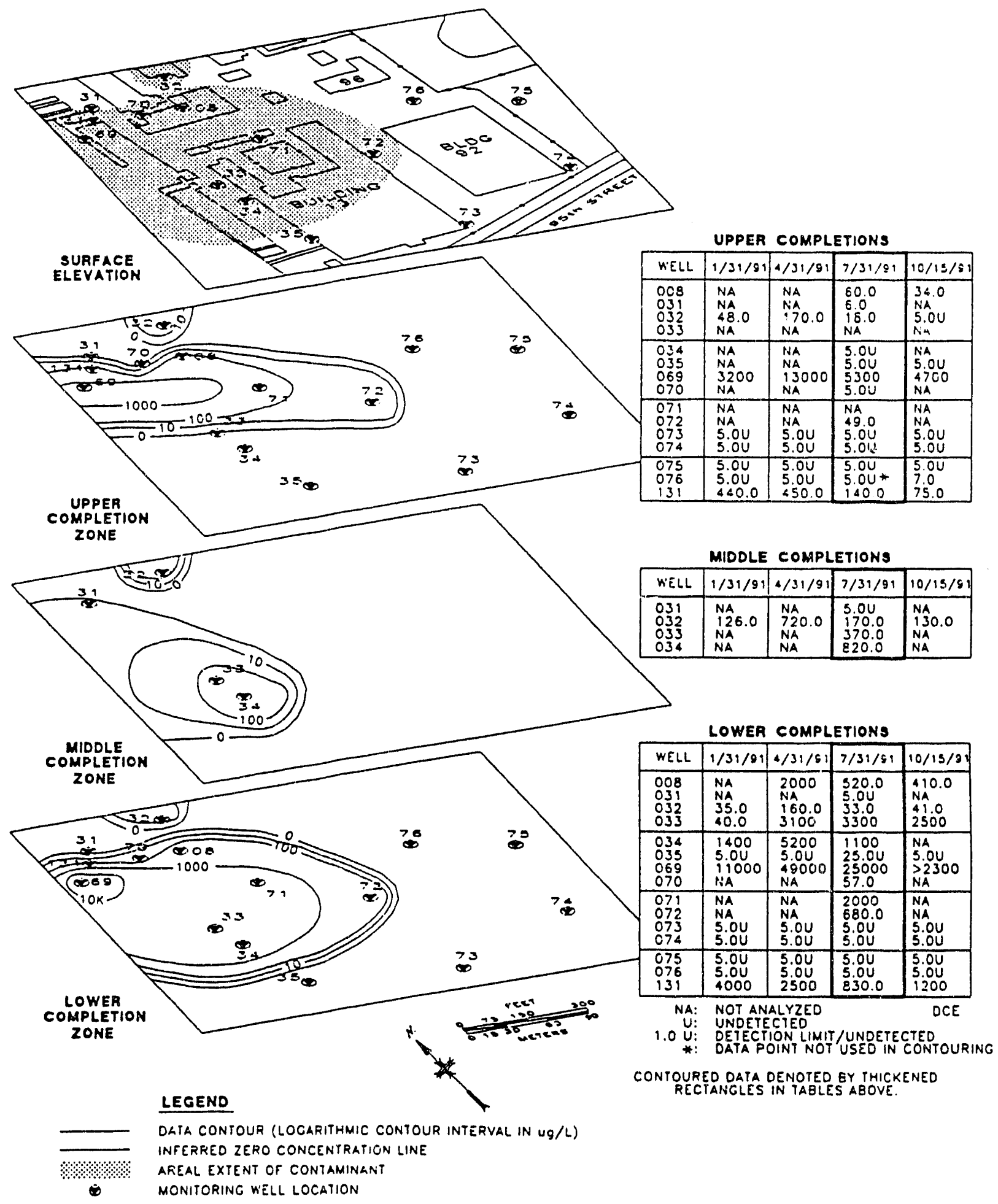

Fig. 8. Depiction of the DCE plume in the TCE Still Area. 
ORNL-DWG 93-5895

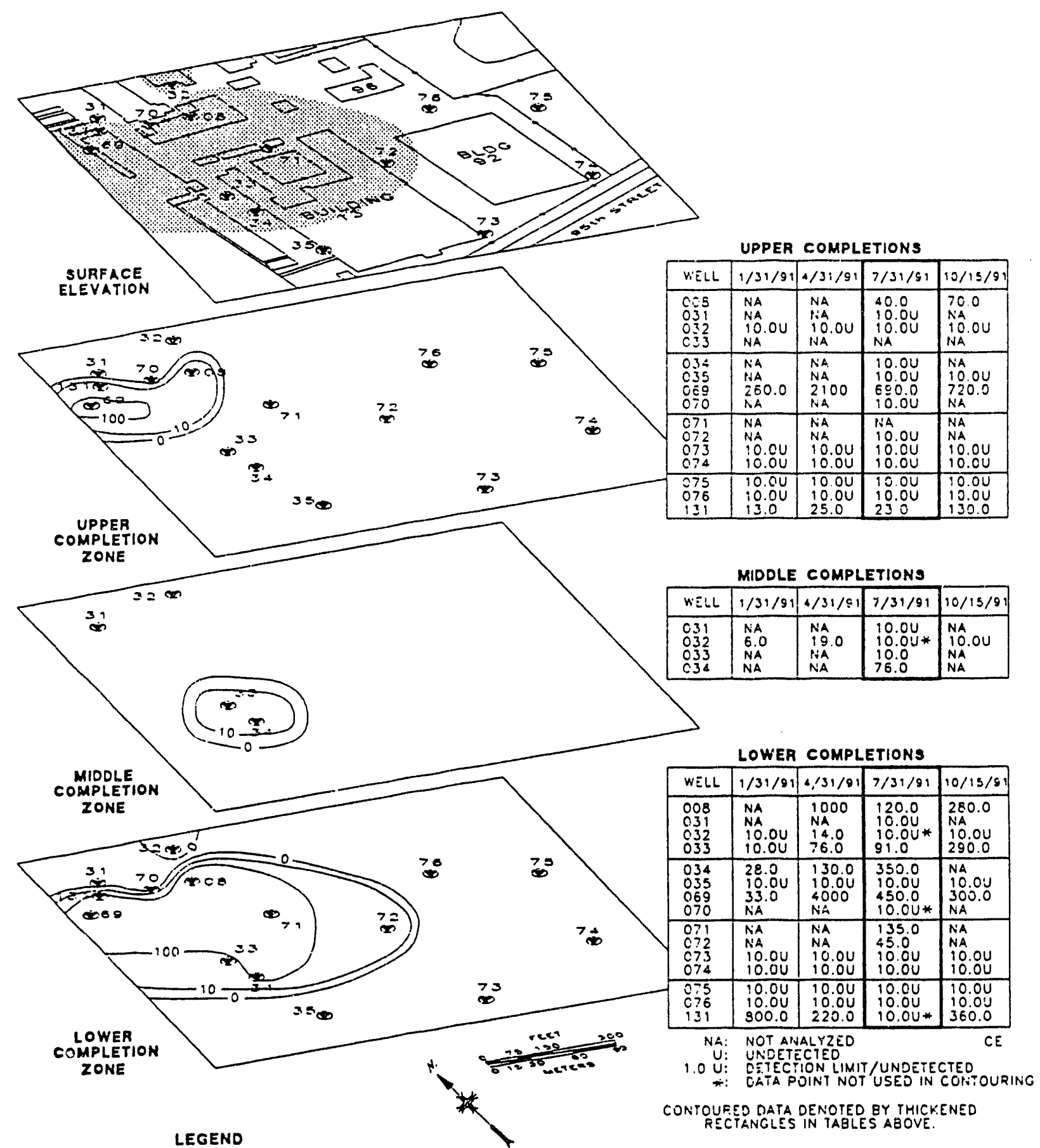

CAIA CONTOUR (LOGRRITHMIC CONTOUR INIERVAL IN UG/L)

CAIA CONTOUR (LOGRRTTHMIC CONTOUR
INFERREO ZERO CONCENTRATION LINE
LAEAL EXTENT OF CONTAMINANT

of NCNITORINC WELLL LOCATIDN

Fig. 9. Depiction of the chloroethene plume in the TCE Still Area. 
major contaminants in the TCE Still Area. Note that monitoring well number 35 , near the lower left corner of the maps, is immediately east of the southeast corner of the Main Manufacturing Building. Figure 2 shows the location of well 35 in relation to the possible location of the old gas wells. Other possible locations of the gas wells are discussed in Sect. 3.2; however, all locations are generally concentrated near the southeast corner of the main manufacturing building.

Other alluvial monitoring wells near the old gas well are shown in Fig. 2. Well KC88-94 contains no contamination. Well KC85-44 contained $76 \mu \mathrm{g} / \mathrm{L}$ of TCE in a sample collected in May 1986; annual samples collected since 1986 have shown no detectable contamination. The lower completion of well KC91-158 contained $6 \mu \mathrm{g} / \mathrm{L}$ of 1,2-DCE (total) in October 1992. Well KC91-164, both upper and lower completions, contained no contamination in January 1992. However, in April 1992, this well contained $9 \mu \mathrm{g} / \mathrm{L}$ and $32 \mu \mathrm{g} / \mathrm{L}$ of $1,2-D C E$ (total) in the upper and lower completions. Well KC91-165, $50 \mathrm{ft}$ downgradient from KC91-164, contained up to $890 \mu \mathrm{g} / \mathrm{L}$ of $1,2-\mathrm{DCE}$ (total) and $1300 \mu \mathrm{g} / \mathrm{L}$ of TCE in the lower completion. The upper completion of well KC91-165 contained $7 \mu \mathrm{g} / \mathrm{L}$ of 1,2-DCE (total).

Near the well labelled \#2 (Fig. 2), wells KC91-159, KC91-161, and KC91-163 contain up to $1800 \mu \mathrm{g} / \mathrm{L}$ volatile organic compounds (VOCs). The upper completion of well $\mathrm{KC} 91-159$ contained $1800 \mu \mathrm{g} / \mathrm{L}$ of $1,2-\mathrm{DCE}$ (total) in a sample collected in April 1992. The levels in other samples in all of the wells are generally below $300 \mu \mathrm{g} / \mathrm{L}$ of various VOCs.

The old natural gas well labelled \#7 is in the TCE Stili Area. Groundwater from well $\mathrm{KC85-33}$ contained $24,000 \mu \mathrm{g} / \mathrm{L}$ of TCE in a sample collected in July 1991. This was the highest level reported in the area since sampling began in 1985. Other VOCs are also present at levels less than those reported above. Historical sample results from these wells are presented in Appendix C. 


\section{OIL AND GAS EXPLORATION AND PRODUCTION}

This section describes the history of oil and gas exploration and production in the Kansas City region near KCP, drilling and abandonment methods of the era, and known information about the natural gas wells at KCP.

\subsection{HISTORY AND METHODS OF THE PRE-WORLD WAR II ERA}

\subsubsection{Regional History}

According to McCourt (1917), drilling began around the Kansas City area in the 1860's. Oil seeps attracted exploration in the area east of Paola, Kansas. The first three wells drilied here were near Wea Creek (SW1/4, Sect. 15, T17S, R23E). The first two were 4-in. wells drilled to $100 \mathrm{ft}$; these wells were dry. The third well was drilled deeper (unspecified) and produced oil. Oil exploration was halted at the start of the Civil War. Figure 10 shows the Kansas City region, with township and range marks for location of various oil and gas fields. Figure 11 shows oil fields in Johnson County, Kans. as of 1954.

Drilling resumed in 1873 with Colonel Acers' "diamond" drilled hole. Total depth drilled was $737 \mathrm{ft}$; the well produced gas and salt water. "Diamond" drilling consisted of a string of hollow steel, flush-jointed rods screwed together in sections. This type of drilling allowed for the extraction of a core of the rock, making exact depths of formations and producing zones much easier to determine (McCourt 1917).

According to the University of Kansas (1908), gas was piped to a zinc smelter (location unspecified) in 1897 and to a Poriland cement plant in 1899. The gas was used to power these industries. Oil had been used previously as a lubricator on freighters' wagons and for domestic medical remedies. According to this article, in the 1880 s, gas wells had been producing for 15 to 20 years, but "possibly for a lack of care, water has drowned them, so that they have lost their value." By 1884, 


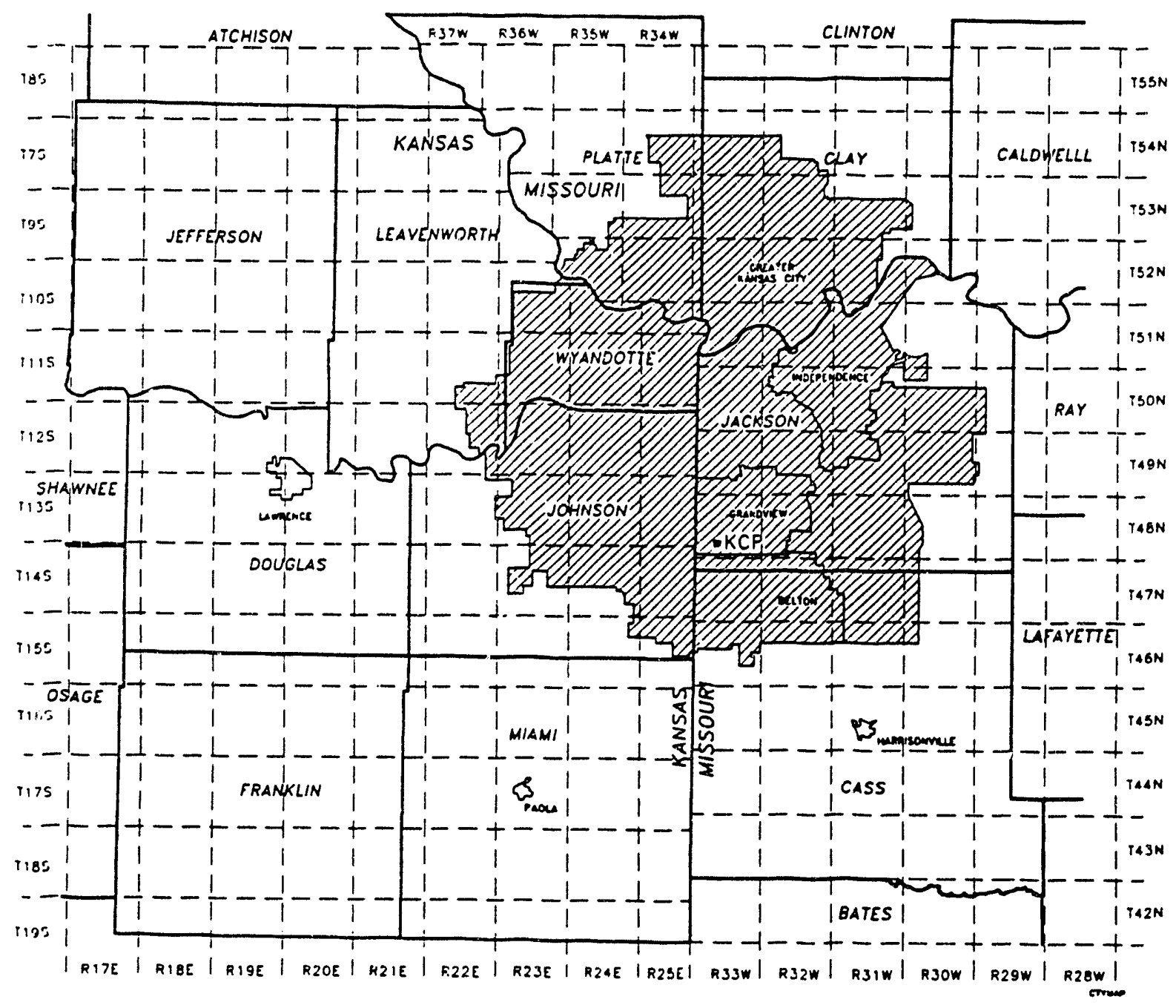

Fig. 10. Location of KCP within greater Kansas City, Jackson County, neighboring counties, and regional townships. 
ORNL-DWG 93-5897

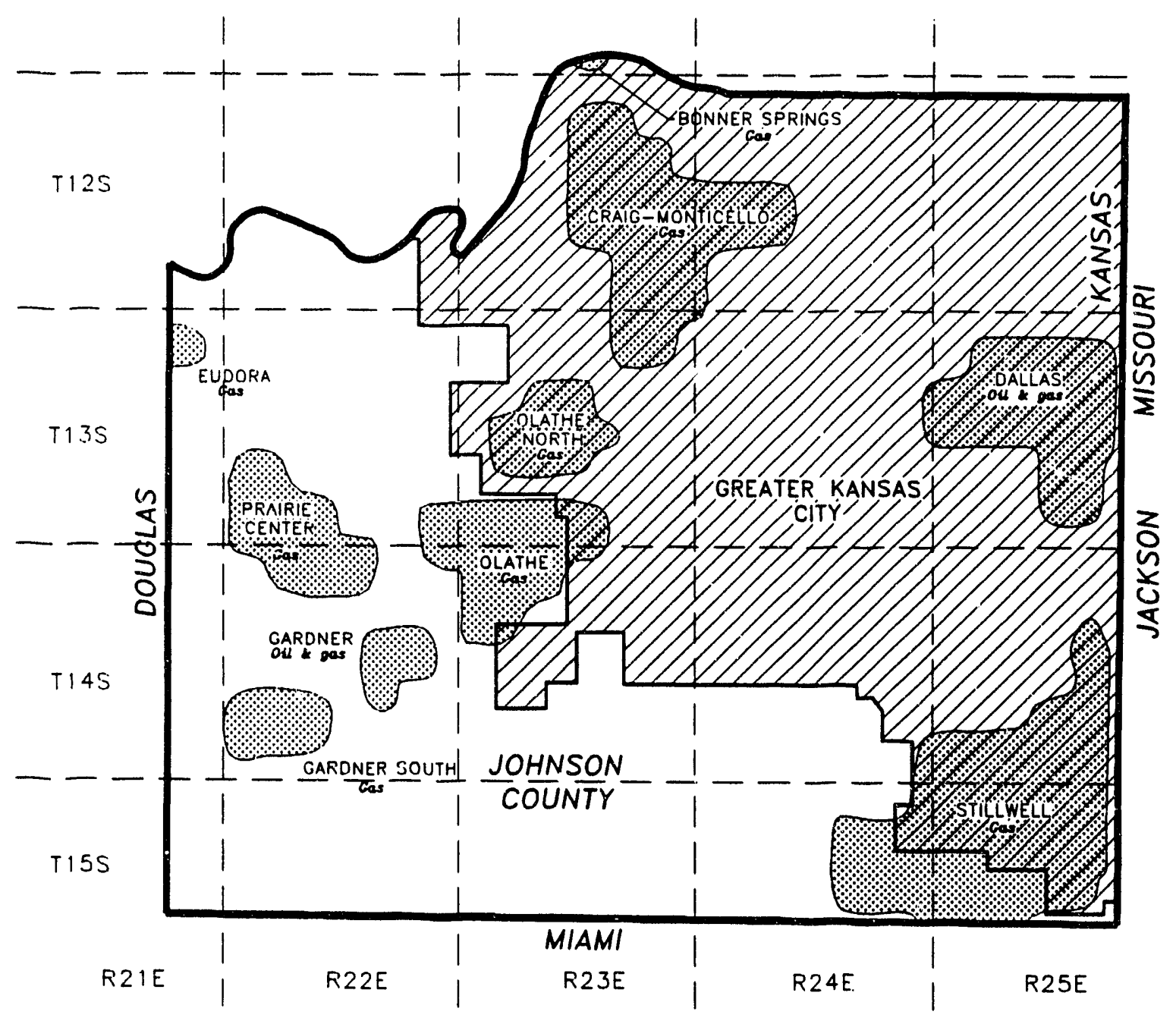

Fig. 11. Oil and gas fields in Johnson County, Kans., in 1954. Source: Jewett, John Mark, and Margaret O. Oros. [1954]. 1979. Oil and Gas in Eastern Kansas with a 25-year Update by Margaret O. Oros. Bulletin 104, reprint. Kansas Geological Survey, University of Kansas, Lawrence, Kans. 
Paola, Kans., had piped gas 7 miles into town from the Paola field. By 1886, the field had a small refinery and, by 1889 , was producing 500 barrels of oil (unit of time was not reported, i.e., per day, week, etc.). By 1910, petroleum exploration in Independence, Mo., and Montgomery County, Kans., had expanded. A Mr. Louis H. Knocke furnished Martin City, Mo., with gas from one well at the rate of 160,000 to $642,000 \mathrm{ft}^{3}$ per month.

Between 1902 and 1906, 15 wells were drilled west and north of Belton, Mo., which is located approximately 6 miles south-southeast of KCP. A Mr. Goodbar supplied approximately 25 families with natural gas from a well $3 / 4$ miles north of Belton. Improper casing techniques destroyed most wells because water and/or sand entered the wells (McCourt 1917). Whether the water was groundwater or surface water was not mentioned. Mineral baths were being developed during this time using water from non-producing gas wells; as a result, numerous wells were purposely drilled for brine cr saltwater.

Promising discoveries close to the Kansas City market prompted wildcat operators to drill in Johnson County, Kans. Wells in Gardener (Sect. 14, T14S, $\mathrm{R} 22 \mathrm{E})$ produced from the Marmaton sandstone. The Craig-Monticello field produced gas from sandstones in the Pleasanton, Marmaton, and Cherokee Groups, with principal production from the upper Cherokee. The field is now used for underground gas storage. In Sect. 15, T12S, R23E, small gas fields were developed in the Knobtown sandstone in the upper part of the Pleasanton shale.

\subsubsection{Local History}

Geologic maps of Jackson County, Mo., from 1922 (Wilson 1922) show a town named Dallas close to the region currently covered by KCP (Fig. 12). Dallas appears on the map two miles south-southwest of the junction of Indian Creek and the Big Blue River. Numerous gas wells had been drilled in this area at the time of the publication (Wilson 1922). Gas was used for domestic and small manufacturing purposes. Depth to the oil and gas varied from 75 to $550 \mathrm{ft}$. Two lithologic logs were included in the article; however, no drilling or completion information was included. The author infers that 110 to 150 wells were drilled, of which 110 were 


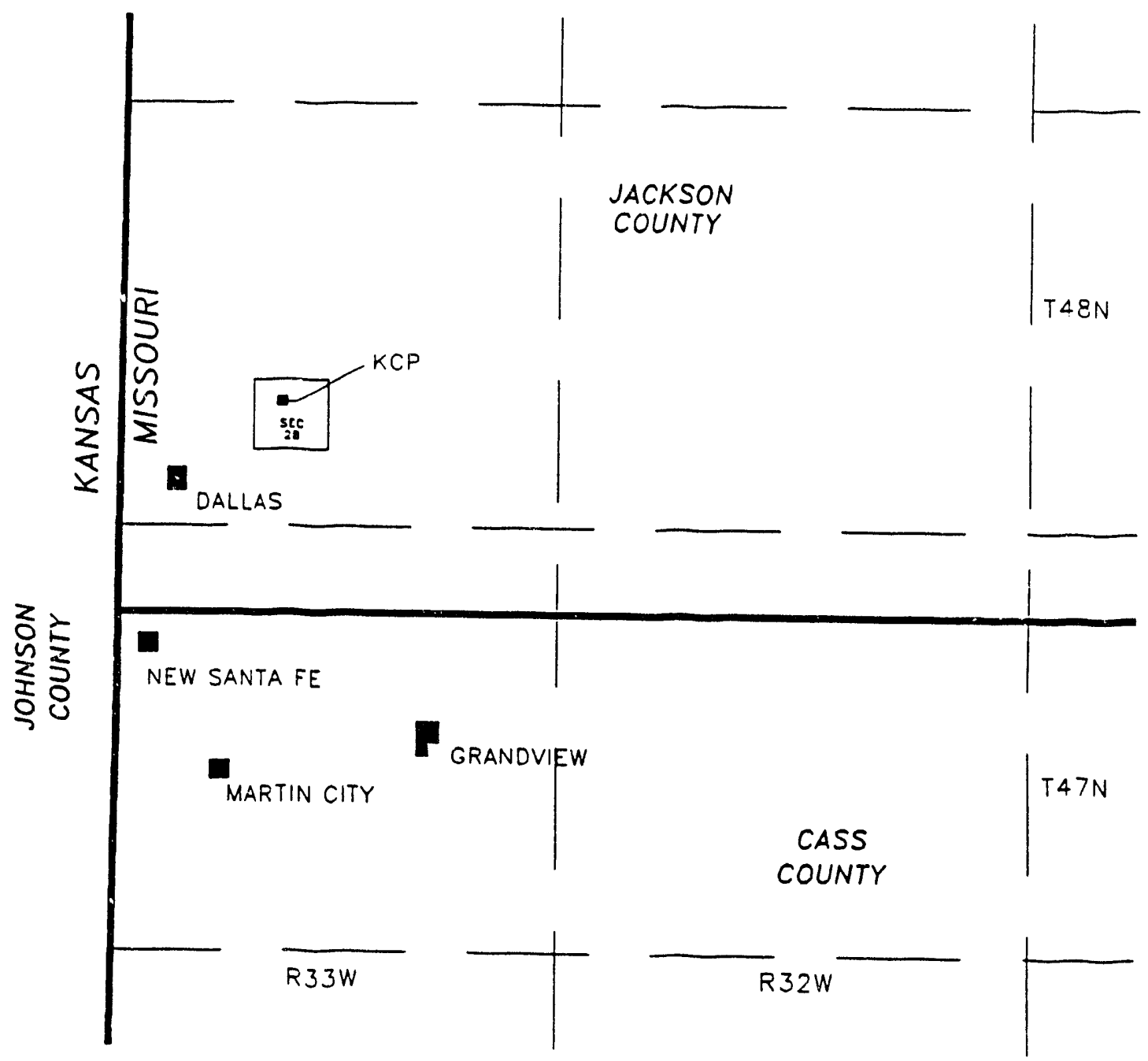

Fig. 12. Location of historical towns close to KCP. 
productive; he further infers that numerous other dry wells were not recorded (Wilson 1922, pp 144-146). "A number of wells were drilled between Dallas and New Santa Fe" (Fig. 12).

At the close of 1927, the Dallas oil field (Miami County, Kans., Sect. 3, 4, 10, 11,14 , and 15, T13S, R25E), had approximately 75 wells. The principal oilproducing horizon was $60 \mathrm{ft}$ of Bartlesville Sand in the upper Cherokee Group. Gas was also produced from a sand in the upper part of the Marmaton Formation. The field had been abandoned during the Civil War, but interest was renewed in 1927 (Kesler 1927).

In 1937, approximately 50 wells were drilled into the Sniabar member of the Hertha Formation, creating the Sniabar gas field in central Jackson County, Mo. This gas freld was near Independence, Mo. (Bartle 1938).

McCuurt (1917) listed eleven wells within a five-mile radius of the present lccation of KCP, all within Jackson County, Mo. The first of these wells was five miles soutt. of KCP (SE1/4, Sect. 20, T47N, R33W), drilled in 1906 by L. H. Knocke. This well, and seven others completed in the area by 1909 , produced natural gas from the middle of the Pleasanton Formation (150 ft), the base of the Henrietta Formation ( $257 \mathrm{ft}$ ), and several depths from the Cherokee shale (274 ft, $365 \mathrm{ft}$, and $\zeta \measuredangle 7 \mathrm{ft}$ ). All depths are reported as feet-below-ground-surface. The best zone was at about $274 \mathrm{ft}$ (Cherokee shale). The third well showed gas sand at $230 \mathrm{ft}$ and oil sands at $287 \mathrm{ft}$ and $421 \mathrm{ft}$ : the first two "shows" were in the Pleasanton and the third in the Cherokee shale (this well was considered a dry hole). The fourth well shows oil sand at $248 \mathrm{ft}$ in the Pleasanton Group and gas sand at $341 \mathrm{ft}$ in the Henrietta Formation. This well was also considered a dry hole. The fifth well shows oil sand at $271 \mathrm{ft}$ in the Pleasanton and gas shale (445 ft) and gas sand (494 ft) in the Cherokee shale.

One well was drilled at Swope Park in 1905 to produce water. Swope Park is approximately two miles northeast of KCP. The well produced gas from 304 to $315 \mathrm{ft}$. No lithologic log was shown. McCourt (1917) also indicates a well drilled at 85th St. and Holmcs Ave. owned by E. Kellerstraus. No date, depth, or lithology information was available. 


\subsubsection{Drilling and Abandonment Methods}

According to Moore and Haynes (1917), cable-tool rigs were frequently used in Kansas and Oklahoma to drill in hard rock formations. The driller would proceed until it was "advisable to case off a water or gas-bearing stratum. In comparatively shallow territory a portable machine of the Star or Parkersburg type was frequently used in the Kansas and Oklahoma fields" (Moore and Haynes 1917). These rigs could be moved easily if roads were bad, but were not adapted for handling heavy strings of cable, thus precluding deep drilling. Drilling consisted of dropping the bit and string of weight repeatedly to break the rock. Cuttings were bailed out of the well. When the well was completed or reached a pay zone in "close-textured reservoir rock, or when the production of a well had begun to decline, it was a common practice to shoot the well, a charge of nitroglycerin being generally used for the shooting" (Moore and Haynes 1917). The amount of explosive varied from a few quarts to 250 quarts.

Cable-tool drilling often included advancing a string of casing as the well was drilled. This casing was advanced as the bit drove deeper intc the rock to prevent caving of sidewalls (UGS 1908). If the blocked water zone was under pressure, the casing was driven or set into a lower layer to securely shut off the water. Occasionally, mud-laden fluid was forced under pressure into the porous rock to form a seal. Further drilling required a smaller bit. Each heavy flow of water was cased off, with each casing set of smaller diameter than the previous casing. Casing was usually sheet-iron riveted pipe of various sizes and weights, depending on the depth of the well and water pressure.

Both cable-tool and rotary drilling rigs were in use in the late 1930 s to early 1940s, according to geological logs from wells (Greene 1945). Typical completions with cable-tool rigs consisted of setting casing at various depths. Each casing interval was followed by smaller diameter casing. A typical well in Platte County, Mo., (drilled in August 1939) was completed by setting 12.5-in. casing to $150 \mathrm{ft}$, 10-in. casing to $931 \mathrm{ft}$, and 8-in. casing to $1103 \mathrm{ft}$. Total depth drilled was $1857 \mathrm{ft}$; the well was dry and was abandoned. 
"The practice of drilling the well and setting the oil string od [sic] casing through the producing zone was universal in the field. Many of the holes were drilled to $100 \mathrm{ft}$ below the deepest possible production in order to allow for flexibility in placing tubing to control gas-oil ratio." (Weeks and Alexander 1942). Producing practices at this time were to set 16 -in. surface casing inside a 21 -in. hole to approximately $100 \mathrm{ft}$, using 125 or more bags of cement. This was followed by setting $103 / 4$-in. casing as intermediate protective casing in a $15 \mathrm{in}$. hole at depths up to and exceeding $2000 \mathrm{ft}$, using 1000 plus bags of cement. Early efforts with shorter protective string were unsatisfactory.

Abandonment of old wells was not documented in any of the literature reviewed. An oil field worker from the pre-World War II era stated that wells were frequently plugged with a cedar tree that had been stripped of its branches. The tree was driven into the hole or casing, followed by cuttings, soil, and/or mud. Although this seems outlandish from a modern perspective, the source was reliable [a Missouri Department of Natural Resources (MDNR) employee for several years], and the method would stop all flow from a low-pressure well. The tree trunk would swell from the moisture, and cedar is very resistant to degradation. Other abandonment procedures were undoubtedly used, probably including no action, leaving drilling mud in the well, and pumping cement into the hole.

The combinations of drilling methods, casing sizes, bit sizes, and cementing practices are limitless. The above examples are a few of the methods described in the literature reviewed; obviously, many combinations were available. Specific methods used in individual wells were commonly not documented. No regulations requiring such documentation were in place. If methods, producing depths, casing sizes, etc., were documented, the information was often a closely held secret; competitors could mobilize onto the neighboring property and drill a well a few feet away. 


\section{KCP NATURAL GAS WELLS}

Information documenting the existence of the old natural gas wells is incomplete. Initial indication that the wells existed came from notes on an old KCP map. This map indicates the locations of 8 wells only near the southeast corner of the Main Manufacturing Building (Fig. 2). Two other sources of information were also discovered: a report by Joseph R. Clair (1943) and records from the MDNR. Clair lists information about seven wells; MDNR records indicate eight wells. Based on location maps (Fig. 2, 13, and 14), the three sources of information appear to be discussing the same wells. Locations were not surveyed but were usually marked by an " $\mathrm{X}$ " within a quarter section drawing. Therefore, the exact location of any well cannot be determined from the descriptions.

Very little information specific to the wells in question was discovered. Registration of wells prior to construction of the plant was apparently voluntary. It information about a well was provided, it was often only a general description of lithology and location.

\subsection{Clair Report, 1943}

KCP is located in Sect. 28, Township 48 North, Range 33 West, Jackson County, Mo. The Clair report (1943) contains two paragraphs of text about this township, data about six wells in Sect. 28, a map showing the probable location of seven wells (scale: 1 in. $=1$ mile) in Sect. 28 (Fig. 13), and a detailed lithologic log from a well in Sect. 36 of the same township. The two paragraphs of text are:

This area is unimportant commercially except for the Indian Creek pool in Sect. 28, Township 48 North, Range 33 West. The wells in this pool were all very small and lasted only a short time. The field lies at the junction of the valleys of Indian Creek and Big Blue River, hence drilling starts in beds stratigraphically below the Kansas City group. For this reason the producing horizon is closer to the surface than in pools under the upland areas. Nine wells have been drilled here, four of 


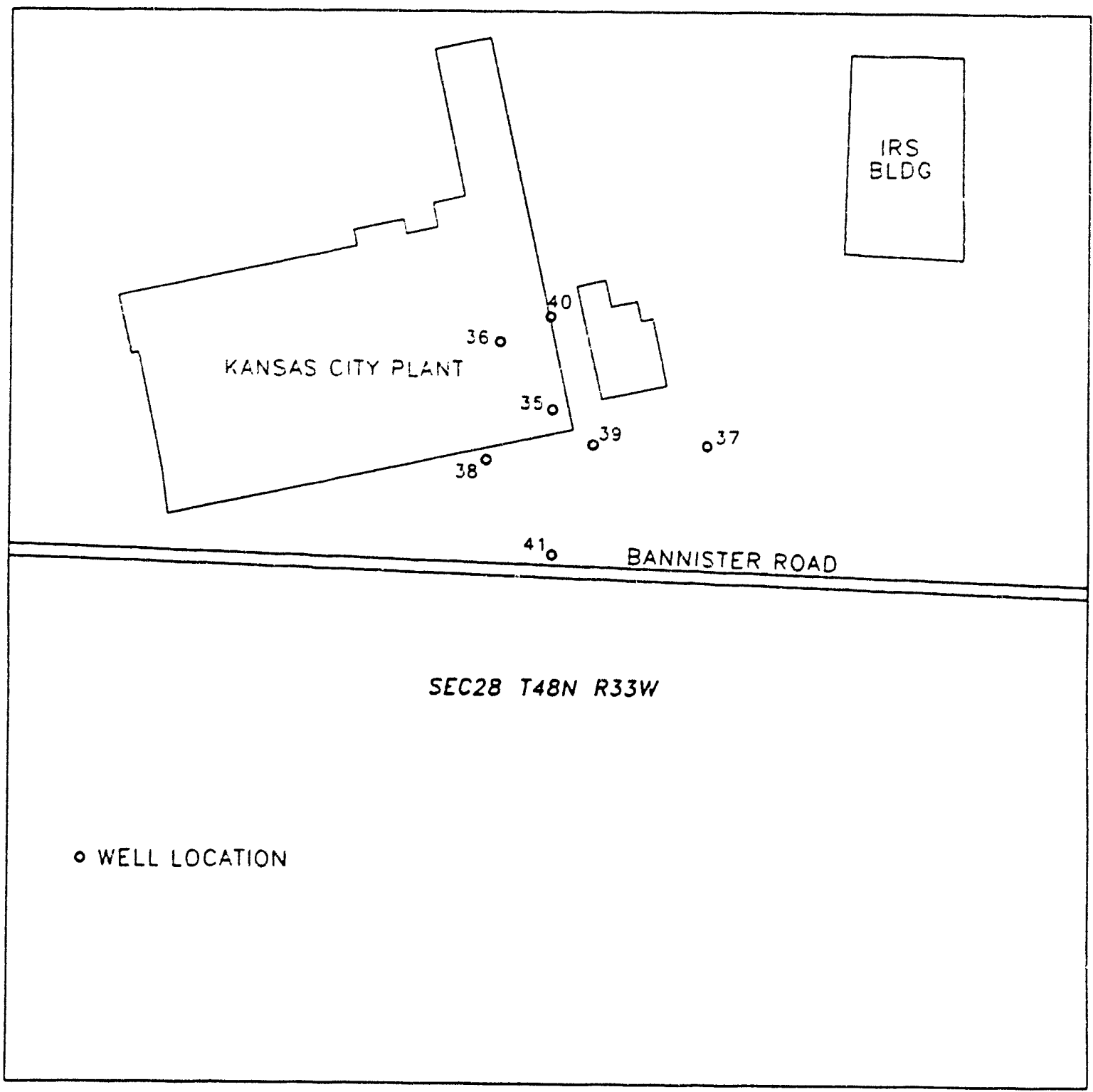

Fig. 13. Location of the seven welks shown on a Clair report map (1943), transposed on a modern map of KCP. 


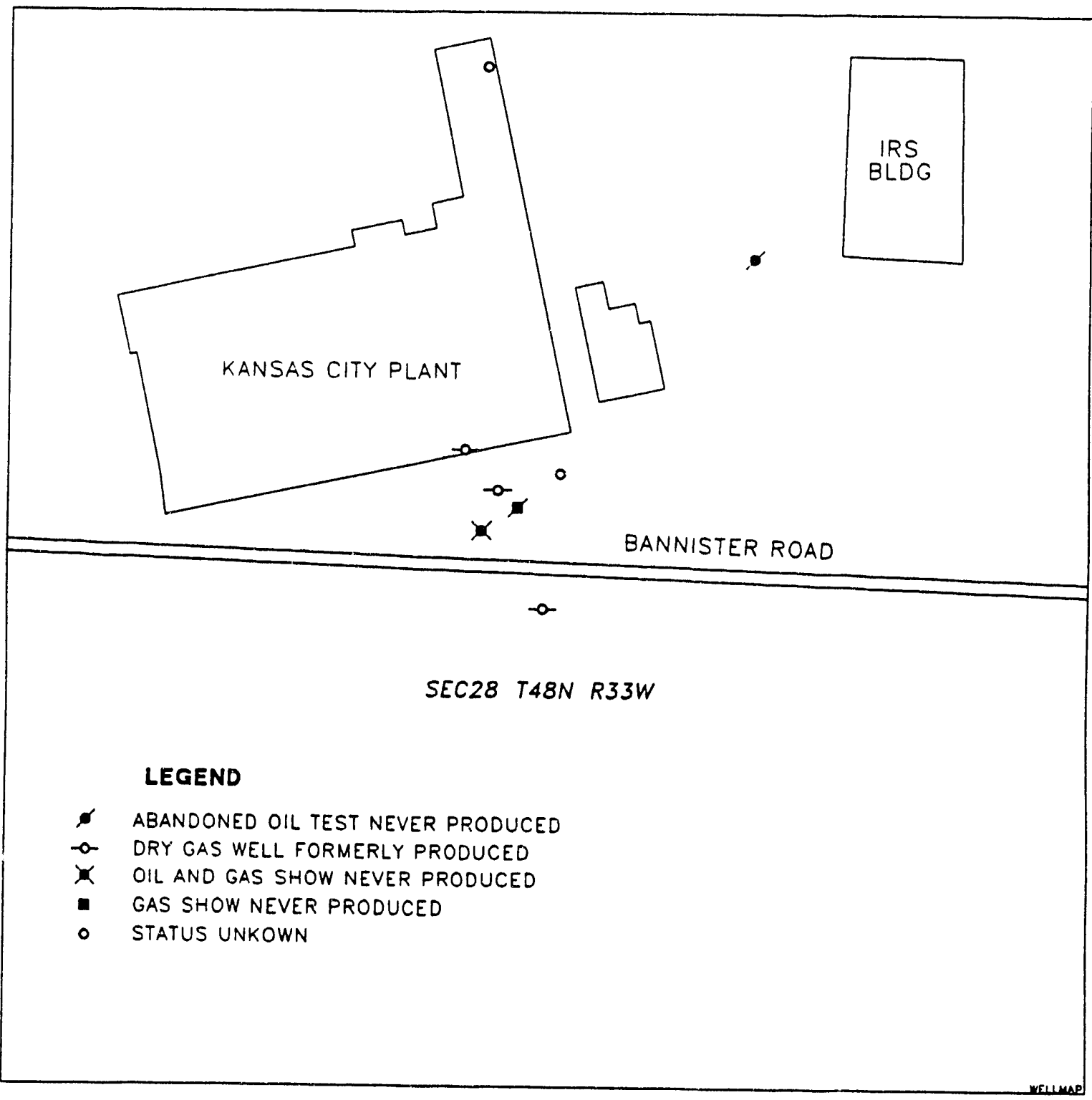

Fig. 14. Location of old wells shown on Missouri Geological Survey map (Netzler 1964) transposed onto modern map of KCP. 
which were dry holes. No information is available on the production and the field is completely abandoned.

There are a number of other wells scattered over the region and several have produced gas. However, none has been of commercial size and they have only been used privately. The future of the region is not very promising and careful detailed mapping should precede any further drilling.

A table later in the same report lists six wells in Sect. 28.

It must be assumed that these six wells in Sect. 28 are part of the seven shown on the map in the report (Fig. 13) and part of the nine discussed in the text. The company for these wells is listed as "Dr. H. H. Francis", the farm and well numbers are listed as "Butler Old Speedway No. 1, 2, 3, 5, 7, and 8". (The $\mathrm{KCP}$ site was formerly the location of a racetrack.) All of the wells are located in Sect. 28, T48N, R33W. Data for the six wells are excerpted from the table and shown below:

$\begin{array}{llllll}\begin{array}{l}\text { Map No. } \\ \text { of well }\end{array} & \begin{array}{l}\text { Surf. } \\ \text { elev. }\end{array} & \begin{array}{l}\text { Total } \\ \text { depth }\end{array} & \begin{array}{l}\text { Depth base } \\ \text { Lex. cap }\end{array} & \begin{array}{c}\text { Elev. base } \\ \text { Lex. cap }\end{array} & \begin{array}{l}\text { Type of } \\ \text { well }\end{array} \\ 35 & 799 & 570 & 181 & 618 & \text { Gas } \\ 36 & 798 & 207 & 195 & 607 & \text { Gas } \\ 37 & 800 & 218 & 206 & 594 & \text { Gas } \\ 38 & 798 & 241 & 200 & 598 & \text { Dry } \\ 40 & 799 & 203 & 198 & 601 & \text { Gas } \\ 41 & 799 & 201 & 198 & 601 & \text { Gas }\end{array}$

The "Lex. cap" used in the table as a depth reference is the Myrick Station limestone of the Pawnee Formation. Used as a marker bed, this limestone was readily identifiable because of the underlying Lexington coal. The Lexington coal 
horizon lies 100 to $210 \mathrm{ft}$ below the Knobtown sandstone (Clair 1943). This correlates well with the depth of the "Lex. cap" in the table, allowing for 10 to $40 \mathrm{ft}$ of alluvium on the Knobtown sandstone. Further assumptions can be made from the information above. The depth of well $35,570 \mathrm{ft}$, indicates that there was apparently little or no show of gas or oil in the lower 300 to $400 \mathrm{ft}$ of the hole since subsequent wells were not drilled beyond approximately $240 \mathrm{ft}$. Gas production was probably in the Lexington coal or a formation immediately above it since all of these wells contacted the coal but none were drilled much deeper.

A single well log from Sect. 36 of the same township was provided in the Clair report (1943). The log is reproduced in Appendix A. This well would be located approximately three miles east-southeast of KCP, with a surface elevation about $230 \mathrm{ft}$ above the elevation of KCP. Due to the relatively flat-lying formations, the top $230 \mathrm{ft}$ of this lithology would not be present in a well drilled at KCP. Interesting notes from the lithologic log are: the Knobtown sandstone was probably thinly bedded with limestone and shale, and the sand went unrecognized; the Lexington cap rock was struck at $430 \mathrm{ft}$; two gas-producing horizons were noted in the Henrietta Group ( $445 \mathrm{ft}$ and $474 \mathrm{ft}$ ); there were two other "shows" of gas and/or oil; and there was no indication of gas or oil below $546 \mathrm{ft}$. No bit sizes or casing information were provided.

\subsubsection{Information from MDNR, 1992}

Records retained by the MDNR also provided some information about eight wells drilled in Sect. 28 of the same township. This information was forwarded to KCP by Bruce Stuart, MDNR, for use in this report.

The records included a Missouri Geological Survey 7.5 minute topographic map of the Grandview Quandragle, Mo.-Kans. quadrangle (Netzler 1964). This map shows the locations of seven wells in Sect. 28 near KCP; Fig. 14 shows the locations of these wells transposed on a modern KCP map. These locations do not match the locations of the wells from the Clair map (1943) or the locations on the old KCP map. However, the locations from all maps are in the same area. 
Locations of the wells were reported to the state only by quarter section and were not surveyed.

The wells on the map are apparently the same wells discussed in the Clair report (1943); MDNR also had copies of old lithologic logs from these wells, listing the owner and well numbers. Seven of these were the same as those in the Clair report (1943). These wells were owned by Dr. H. H. Francis, and the firm was "Butler Old Speedway" or just "Old Speedway". One other well in Sect. 28 is also included. The owner was "Ruf Drlg. Co.," and the farm was "Butler \& Reynolds". All of the Dr. H. H. Francis wells were drilled in the fall of 1933; the Ruf Drlg. Co. well was drilled in June 1936.

Because the eight lithologic logs are nearly illegible, highlights from each of the eight logs are provided below. The comments shown by the depths are notable remarks from each log. Exact quotes are shown; question marks were used where a word was unreadable. No information was reported about abandonment or plugging of the wells. Our comments appear in brackets.

\subsubsection{Information from KCP Employee}

A KCP employee who had been at the plant since 1949 recalled information about one of the old gas wells. This employee is now retired. During the late 1950 s or early 1960 s, one of the old wells was leaking gas. A contractor (Apparently Layne-Western) sealed the well using "present" technology (Floyd Bowlin, Allied Signal, Inc., Kansas City, Mo., personal communication to D. E. Brown, Allied Signal, Inc., 1992). The leaking well was located near the "Q-tunnel head house". A well near the Q-tunnel head house would match the location of well \#7 from the old KCP map (Fig. 2) and well \#40 from Clair (1943) (Fig. 13). The area near the Q-tunnel head house is covered with asphalt and/or concrete; no surface expression of the well is present. 
Owner: Ruf Drlg. Co. Farm: Butler \& Reynolds Well No. 1

Date: June 3,1936 Casing record: set 6 1/4" at 50' Elevation: 798

Depth

49 Mud and gravel, Water - set $61 / 4^{\prime \prime}$ at 50'

198 Lime (Lex. Cap)

201 Shale, black about 8 to 10,000 gas

265 Sand - oil showing

314 Sand, broken, dark, oil show

340 shale, TD with $61 / 4^{\prime \prime}$ bit

[The well was drilled to total depth with a $61 / 4$ " bit, indicating that no further casing was set below the $50 \mathrm{ft}$ of surface casing. The lithology was based on the driller's log.] 
Owner: H. H. Francis

Farm: Old Speedway Well No.

Date: August 1933

[Remarks are mostly unreadable; some interpretation was required for the following highlights.]

\section{Depth}

$0-171 \mathrm{ft}$

unreadable

181

went about 3???????

188

Lexington Coal ???????? full of salt water

206

Oil showing

277

Squirrel

307

oil showing

347

oil ??????? showing

389

$47 / 8$ " casing ?????????

570

Burgess oil showing

TD

To be termed as gas well

[The "Squirrel" is probably the Squirrel sand in the upper Cherokee Group; the "Burgess" is probably the Burgess sand of the lower Cherokee Group.] 
Owner: H. H. Francis

Farm: Butler (Speedway). Well No. 2

Date: August 1933 Driller: Rand G. Bradford? Elevation: 798.0

Depth

10 - 50

rivermud

heavy water

93

black ???! gas ???????

200

black. Lex. coal horizon

207

bottom of sand - oil sand

TD

Packer set at 194'

Owner: Dr. H. H. Francis

Farm: Butler (Speedway)

Well No. $\underline{3}$

Date: 1933

Driller: Raymond Bradford?

Elevation: $\underline{799.5}$

Depth

$20-41$

41

river mud sand

148

212

water $41^{\prime}$ of $81 / 4^{\prime \prime}$ pipe

set 80 ' of $81 / 4^{\prime \prime}$ pipe

218 dark gray measured hole

little gas - water

sand oil [last entry, apparent TD at $218 \mathrm{ft}$ ] 
Owner: (unreadable)

Date: Fall 1933
Farm: Old Speedway

Elevation: $\underline{798.3}$
Well No. $\underline{5}$

Depth

0 - 147

[no entries]

147

set $61 / 4^{\prime \prime}$

192

sand

202

gas bubbles

241

no gas [last entry, apparent TD]

Owner: Francis Dr.

Farm: Old Speedway

Well No. 6

Date: Fall 1933

Driller: Ed Feyh (contr) Elevation: 799

Depth

$0-33$

soil and clay

42

$41^{\prime}$ of $81 / 4^{\prime \prime}$

187

[last entry, apparent TD]

[There were no entries on this log other than those shown, plus some intermediate depths with no comments.] 
Owner: Dr. Francis

Farm: Old Speedway

Well No. 7

Date: Fall 1933

Driller: Ed Feyh (contr) Elevation: 799

\section{Depth}

10 soil

203 [last entry, apparent TD]

[The only comments on this log were references to color; no lithology was described.]

Owner: Dr. Francis

Date: Fall 1933
Farm: Old Speedway

Driller: Ed Feyh (contr.)
Well No. 8

Elevation: $\underline{798.6}$

Depth

42

161

170

41' of $81 / 4^{\prime \prime}$ pipe

171

162163 Little gas-water

???

packer set 169 1/2

183

gas

gas

201

water

[last entry, apparent TD] 


\section{DISCUSSION}

Eight abandoned gas wells are reported to be located on KCP property. No abandonment records exist for the wells. The best case scenario assumes that the wells were plugged, and no hydraulic connection between water table and bedrock aquifers could exist. The worst case scenario assumes that the gas wells were not properly plugged or have not collapsed and act as conduits between the alluvial and the bedrock aquifers. Some conclusions that can be drawn from the available data are presented below.

\subsection{HYDROLOGIC}

If a downward, vertical hydraulic gradient is present between the alluvial and bedrock aquifers in the vicinity of the abandoned gas wells, groundwater from the alluvial aquifer would migrate down the abandoned gas wells and discharge to the bedrock. Conversely, if an upward vertical hydraulic gradient is present between the bedrock and alluvial aquifers in the vicinity of the abandoned gas wells, groundwater from the bedrock would discharge to the alluvial aquifer. Therefore, in the absence of any well abandonment information, the question of whether contaminated groundwater from the alluvial aquifer is discharging to the bedrock aquifer through the abandoned gas wells can be answered by determining the direction of the vertical hydraulic gradient between these aquifers in the vicinity of the abandoned gas wells.

No bedrock monitoring wells are located in the immediate vicinity of the abandoned gas wells from which water-level elevation data can be obtained and compared with existing alluvial water-level data to determine the direction of the verticai hydraulic gradient between the two aquifers. However, examination of bedrock stratigraphy and regional direction of dip provides some insight into the probable direction of the vertical hydraulic gradient in the vicinity of the abandoned gas wells. 
Regional groundwater flow in the bedrock beneath KCP is controlled by bedrock dip direction and stratigraphic sequence. Bedrock beneath the KCP dips gently to the northwest (Fig. 5). Bedrock units below the Knobtown sandstone subcrup or outcrop east of KCP. Recharge occurs where bedrock units subcrop or outcrop. Groundwater in the more-permeable bedrock units flows down-dip from the recharge zones and becomes confined by the overlying less-permeable shale units. A short distance down-dip, along the flowpath, the groundwater potentiometric surface of the bedrock unit intersects the groundwater potentiometric surface of the alluvium, resulting in an upward vertical hydraulic gradient.

Water-level data is available for bedrock monitoring well $\mathrm{KC} 84-023$, screened in the Hepler sandstone. Alluvial monitoring wells KC84-015, KC84-016, KC84-017, and KC85-042 are located adjacent to KC84-023 (Fig. 3). Historical water-level data for these wells are presented in Appendix B. Comparison of average water-level elevations for the five wells demonstrates that the hydraulic gradient is upward between the alluvial and Hepler sandstone aquifers. The abandoned gas wells were completed in bedrock units located below the Hepler sandstone. Because the recharge areas for these units are located further east than the Hepler recharge area, the vertical hydraulic gradient between these lower bedrock units and the alluvium should also be upward. Consequently, because of the upward vertical hydraulic gradient, contaminants found in the alluvial aquifer could not migrate down the abandoned gas wells and contaminate the bedrock aquifer.

\subsection{STRATIGRAPHIC}

The Knobtown and Hepler sandstones are both low-permeable formations (Sect. 2.2.3). The Pleasanton shale has been shown to be nearly impermeable and unfractured. Horizontal migration of contaminants in any of these formations would be extremely slow; vertical migration would be even slower. If contaminants were intermixing with bedrock groundwater as a result of one of the gas wells, transport would be minimal. 
The first $100 \mathrm{ft}$ or more of the underlying formations are represented by the Pleasanton Group, which are relatively impermeable and would act as barriers to further transport. Below the Pleasanton Group lie 150 to $300 \mathrm{ft}$ of the Marmaton Group, which consists of limestone and shale with the Lexington coal and one sand lens near the middle. The Lexington coal was not present in the well in Sect. 36, but the cap rock (Myrick limestone) was logged (Appendix A). [Note: Between 1943 and the early 1960s, formations in the Henrietta and Cherokee Groups were apparently regrouped and the former Henrietta Group was renamed the Marmaton Group. Modern designations are used in this report, but the well logs use the designations accepted at the time the well was drilled.] Below the Marmaton lie 300 to $400 \mathrm{ft}$ of the Cherokee Group. In the well in Sect. 36, the Cherokee consisted of mostly shales with a few sand and limestone layers (Clair 1943). Therefore, most of the first several hundred feet of stratum underlying the plant are relatively impermeable, with scattered more-permeable zones. The shales and limestones of these groups are relatively unfractured.

\subsection{HISTORICAL}

The logs from the wells owned by Dr. H. H. Francis (Sect. 3.2.2) show that casing was set through the alluvium in most of the wells. Casing was probably set in all of the wells. However, this information was not recorded on the logs or the logs are unreadable. Thus, a physical barrier was installed that would slow the flow of contaminants from the alluvial groundwater. The quality of the barrier at the time of installation is unknown. Casing which remained in the ground would have deteriorated and may have collapsed. If casing through the alluvium has collapsed, the wells have undoubtedly sealed with the silty clays common in the alluvial system.

The wells were apparently producing natural gas from depths of approximately $200 \mathrm{ft}$. According to Clair (1943), they were not prolific and were abandoned only a few years after drilling. Since the wells produced only small quantities of gas for a few years, the gas was probably at low pressure. After a well was 
abandoned, water would effectively seal off the low pressure gas from migrating to the surface. Collapse of a well would place a final seal on the gas-producing horizons.

\subsection{RECOMMENDATIONS}

No search for or further consideration of the old gas wells is recommended. The most likely scenario is that the wells were either sealed or have collapsed. Furthermore, the hydrology of the area probably creates an upward vertical flow. Even if the wells do remain open, the upward vertical gradient prevents contaminants from migrating down the well annulus.

Most of the possible locations of the old gas wells are presently covered with structures or parking lots. Locations not covered by structures have been heavily disturbed several times during activities at the KCP. These activities include rerouting of Indian Creek, burial of numerous utilities, construction and subsequent demolition of the lagoons, and modification of traffic patterns and parking lots.

Discussions with geophysicists indicate that geophysical techniques would probably not locate the wells (J. E. Nyquist, Oak Ridge National Laboratory, Oak Ridge, Tenn., personal communication to N. E. Korte, Oak Ridge National Laboratory, Grand Junction, Colo., 1992), especially without accurate information about the location of the old wells. Three sources of information (KCP map, Clair [1943], and MDNR) place the wells within several hundred feet of each other. Although this is valuable information indicating that the wells existed near the plant, it is insufficient when attempting to locate some remnant of a well using a remote sensing technique such as geophysics. The profusion of buried and aboveground utilities make geophysical techniques difficult at best, and without some knowledge of the target, the problem becomes insurmountable. 


\section{REFERENCES}

Bartle, Glenn C. 1938. Subsurface study of Cherokee Formation near Kansas City, Mo. Bulletin of AAPG, 22(2).

Clair, Joseph R. 1943. The Oil and Gas Resources of Cass and Jackson Countries, Missouri. Vol. XVII, second series. Missouri Geological Survey and Water Resources, Rolla Mo.

Fleischhauer, H. L., N. Korte, S. Sturm, S. Wagner, and J. M. Sewell. 1986.

Hydrogeologic Site Characterization of the Department of Energy Kansas City Facility: Second Interim Report. Report No. GJ-40. Bendix Field Engineering Corp., Grand Junction, Colo.

Gann, E. E., E. J. Harvey, J. H. Barks, D. L. Fuller, and D. E. Miller. 1974.

Water Resources of West-Central Missouri. Hydrologic Investigations Atlas, HA-491. U. S. Geological Survey.

Greene, Frank C. 1945. Recent Drilling in Northwestern Missouri, Report of Investigations, No. 1. Missouri Geological Survey and Water Resources, Rolla, Mo.

Heckel, Philip H. 1978. Field Guide to Upper Pennsylvanian Cyclothemic Limestone Facies in Eastern Kansas. Guidebook Series 2, Kansas Geological Survey, University of Kansas, Lawrence, Kans.

Jewett, John Mark, and Margaret O. Oros. [1954]. 1979. Oil and Gas in Eastern Kansas With a 25-year Update by Margaret O. Oros. Bulletin 104, reprint. Kansas Geological Survey, University of Kansas, Lawrence, Kans.

KCD. 1987. Kansas City Plant Site Development Plan. BDX-613-2967R.

Revised. Allied-Signal, Inc., Kansas City Division, Kansas City, Mo.

Kesler, . W. 1927. Oil and gas resources of Kansas in 1927. Mineral Resources, Circular 1, 29(11), QE113, M46, no. 1.

Korte, N. E., P. M. Kearl, H. L. Fleischhauer, and J. M. Sewell. 1985.

Hydrogeologic Characterization of the Department of Energy Facility: Interim Report. Report No. GJ-31. Bendix Field Engineering Corp., Grand Junction, Colo. 
Madril, M. W., M. R. Meininger, S. M. Sewell, P. R. Engelder, P. M. Kearl, R. J. Zinkl, N. E. Korte, and H. L. Fleischauer. 1986. Hydrogeological Site Characterization of the Kansas City Department of Energy Facility: Hydrogeology and Contaminant Transport Modeling. Report No. GJ-46. Bendix Field Engineering Corp., Grand Junction, Colo.

McCourt, Walter Edward. 1917. The Geology of Jackson County. Vol. XIV, second series. Missouri Bureau of Geology and Mines, Rolla, Mo.

Moore, Raymond C., and Winthrop P. Haynes. 1917. Oil and Gas Resources of Kansas. Bulletin 6, Part 1. Kansas State Geological Survey, Lawrence, Kans., pp. 1-83.

Netzler, Bruce W. 1964. Missouri Oil and Gas Wells of Record, 1860 - Present (August 1987). Grandview Quadrangle, Missouri-Kansas, 7.5 minute series topographic map. OFM-82-90-OG. Missouri Geological Survey, Rolla, Mo.

UGS. 1908. Special Report on Oil and Gas, Vol. IX. The University Geological Survey of Kansas, University of Kansas, Lawrence, Kans.

U.S.DOE. 1984. Preliminary Hydrogeologic Investigation of the DOE Kansas City Facility (revised). U. S. Department of Energy, Grand Junction Area Office, Grand Junction, Colo.

U.S.DOE. 1990a. Annual Groundwater Monitoring Report. U.S. Department of Energy, Kansas City Area Office, Kansas City, Mo.

U.S.DOE. 1990b. Groundwater Quality Assessment Plan for the Northeast Area.

U. S. Department of Energy, Kansas City Area Office, Kansas City, Mo.

U.S.DOE. 1990c. Groundwater Quality Assessment Plan for the Tank Farm Area. U. S. Department of Energy, Kansas City Area Office, Kansas City, Mo.

U.S.DOE. 1990d. Groundwater Quantity Assessment Plan for the TCE Still Area.

U. S. Department of Energy, Kansas City Area Office, Kansas City, Mo.

U.S.DOE. 1990e. Groundwater Quality Assessment Plan for the Miscellaneous

Sites. U. S. Department of Energy, Kansas City Area Office, Kansas City, Mo.

U.S.DOE. 1991a. Annual Groundwater Monitoring Report. U. S. Department of Energy, Kansas City Area Office, Kansas City, Mo. 
U.S.DOE. 1991b. South Lagoon RCRA Facility Investigation Report. U. S. Department of Energy, Environmental Restoration Program, Albuquerque Operations Office, Albuquerque, N.M.

U.S.DOE. 1991c. TCE Still Area Resource Conservation and Recovery Act (RCRA) Facility Investigation Work Plan. U. S. Department of Energy, Kansas City Area Office, Kansas City, Mo.

U.S.DOE. 1992a. Groundwater Interceptor System Evaluation. U. S. Department of Energy, Environmental Programs Branch, Albuquerque Operations Office, Albuquerque, N.M.

U.S.DOE. 1992b. Miscellaneous Sites RCRA Facility Investigation Report. Draft. U. S. Department of Energy, Environmental Programs Branch, Albuquerque Operations Office, Albuquerque, N.M.

Weeks, Warren B, and Clyde W. Alexander. 1942. Schuler Field, Union County, Arkansas. Bulletin of the American Association of Petroleum Geologists, 26(9):1467-1516.

Wilson, M. E. 1922. The Occurrence of Oil and Gas in Missouri. Vol. XVI, second series. Missouri Bureau of Geology and Mines, Rolla, Mo.

VerWiebe, W. A., G. E. Abernathy, J. M. Jewett, and E. K. Nixon. 1948. Oil and Gas Developments in Kansas During 1947. Bulletin 78, State of Kansas, University of Kansas Publication. 


\section{ACRONYMS AND INTTIALISMS}

1,2-DCE
DOE
KCP
MDNR
TCE
VOC

1,2-dichloroethene

Department of Energy

Kansas City Plant

Missouri Department of Natural Resources

trichloroethene

volatile organic compound 


\section{APPENDIX A}

Lithologic log of a well in Sect. 36,

T48N, R33W, Jackson County, Missouri 
Log of Russell et al. No. 1, Bannister. Location: Southwest comer NW. 1/4 NE 1/4 NE. 1/4 sec. 36, T. 48 N., R. 33 W. Jackson County, Missouri. Elevation: 1,028.5 feet. Completed: Dec. 4, 1934.

\begin{tabular}{|c|c|c|}
\hline Stratum & $\begin{array}{c}\text { Thickness, } \\
\text { ft }\end{array}$ & $\begin{array}{c}\text { Depth, } \\
\mathrm{ft}\end{array}$ \\
\hline \multicolumn{3}{|l|}{ Quatemary system: } \\
\hline Soil, yellow & 16 & 16 \\
\hline Gravel and water & 2 & 18 \\
\hline \multicolumn{3}{|l|}{ Pennsylvanian system: } \\
\hline \multicolumn{3}{|l|}{ Kansas City Group: } \\
\hline Lime & 4 & 22 \\
\hline Shale, blue & 4 & 26 \\
\hline Lime, light & 7 & 33 \\
\hline Shale, blue & 12 & 45 \\
\hline Lime, light & 10 & 55 \\
\hline Shale, dark & 12 & 67 \\
\hline Shale, red & 8 & 75 \\
\hline Lime, light & 6 & 81 \\
\hline Shale, dark & 4 & 85 \\
\hline Lime, light & 16 & 101 \\
\hline Shale, blue & 25 & 126 \\
\hline Lime, light (Winterset) & 44 & 170 \\
\hline Shale, dark & 4 & 174 \\
\hline Lime, light (Bethany Falls) & 16 & 190 \\
\hline Shale, black & 4 & 194 \\
\hline Lime, light (Hertha) & 14 & 208 \\
\hline \multicolumn{3}{|l|}{ Pheasanton Formation: } \\
\hline Shale, black & 3 & 211 \\
\hline Lime, light & 11 & 222 \\
\hline
\end{tabular}


Log of Russell et al. No. 1, Bannister. Location: Southwest corner NW. 1/4 NE. 1/4 NE 1/4 sec. 36, T. 48 N., R. 33 W. Jackson County, Missouri.

Elevation: 1,028.5 feet. Completed: Dec. 4, 1934.

\begin{tabular}{|c|c|c|}
\hline Stratum & $\begin{array}{l}\text { Thickness, } \\
\text { ft }\end{array}$ & $\begin{array}{l}\text { Depth, } \\
\text { ft }\end{array}$ \\
\hline Shale, light & 89 & 311 \\
\hline Lime, light & 4 & 315 \\
\hline Sand (Wayside), show of oil & 14 & 329 \\
\hline Shale, blue & 56 & 385 \\
\hline \multicolumn{3}{|l|}{ Henrietta Group: } \\
\hline Lime, light & 10 & 395 \\
\hline Shale, dark & 10 & 405 \\
\hline Lime, light & 5 & 410 \\
\hline Shale, light & 5 & 415 \\
\hline Shale, dark & 11 & 426 \\
\hline Lime, light (Lexington cap rock) & 4 & 430 \\
\hline Shale, sandy & 5 & 435 \\
\hline Sand, $80,000 \mathrm{fi}^{3}$ gas & 10 & 445 \\
\hline Shale, light & 15 & 460 \\
\hline Shale, pink & 4 & 464 \\
\hline Shale, dark & 8 & 472 \\
\hline Shale, black, $100,000 \mathrm{ft}^{3}$ gas & 2 & 474 \\
\hline Shale, light & 16 & 490 \\
\hline \multicolumn{3}{|l|}{$\begin{array}{l}\text { Cherokee Group: } \\
\text { Henrietta-Cherokee contact (approx): }\end{array}$} \\
\hline Shale, black & 10 & 500 \\
\hline Shale, light & 13 & 513 \\
\hline Sand (Squirrel, show of gas and oil) & 33 & 546 \\
\hline Shale, dark & 104 & 650 \\
\hline Shale, white, soft & 15 & 665 \\
\hline
\end{tabular}


Log of Russell et al. No. 1, Bannister. Location: Southwest corner NW. 1/4 NE 1/4 NE 1/4 sec. 36, T. 48 N., R. 33 W. Jackson County, Missouri. Elevation: 1,028.5 feet. Completed: Dec. 4, 1934.

\begin{tabular}{|c|c|c|}
\hline Stratum & $\begin{array}{l}\text { Thickness, } \\
\text { ft }\end{array}$ & $\begin{array}{l}\text { Depth, } \\
\text { ft }\end{array}$ \\
\hline Shale, blue & 5 & 670 \\
\hline Shale, white & 4 & 674 \\
\hline Lime & 4 & 678 \\
\hline Sand and water, stands $500 \mathrm{ft}$ from top & 8 & 686 \\
\hline Shale, dark & 22 & 708 \\
\hline Shale, light & 17 & 725 \\
\hline Sand (black slate one $\mathrm{ft}$ ) & 7 & 732 \\
\hline Shale, gray & 13 & 745 \\
\hline Shale, dark sandy & 49 & 794 \\
\hline Lime, dark, hard & 3 & 797 \\
\hline Shale, dark, sandy, hard & 18 & 815 \\
\hline \multicolumn{3}{|l|}{$\begin{array}{l}\text { Mississippian system: } \\
\text { Meramec Group: } \\
\text { Warsaw Formation: }\end{array}$} \\
\hline $\begin{array}{l}\text { Limestone, gray, white, dense to crystalline, } \\
\text { shaly and glauconitic in lower part }\end{array}$ & 98 & 913 \\
\hline \multicolumn{3}{|l|}{$\begin{array}{l}\text { Osage Group: } \\
\text { Keokuk and Burlington Formations: }\end{array}$} \\
\hline $\begin{array}{l}\text { Limestone, gray, white, cherty, crystalline, } \\
\text { thin dolomite limestone beds in upper part }\end{array}$ & 173 & 1,086 \\
\hline \multicolumn{3}{|l|}{ Sedalia-Reeds Spring Formation: } \\
\hline Limestone, dolomite, tan white to gray, cherty & 166 & 1,252 \\
\hline \multicolumn{3}{|l|}{ Fern Glen Formation: } \\
\hline Limestone, gray, dense & 20 & 1,272 \\
\hline \multicolumn{3}{|l|}{$\begin{array}{l}\text { Kinderhook Group: } \\
\text { Northview Formation: }\end{array}$} \\
\hline Shale, green, calcareous & 10 & 1,282 \\
\hline
\end{tabular}


Log of Russell et al. No. 1, Bannister. Location: Southwest corner NW. 1/4 NE. 1/4 NE. 1/4 sec. 36, T. 48 N., R. 33 W. Jackson County, Missouri.

Elevation: 1,028.5 feet. Completed: Dec. 4, 1934.

\begin{tabular}{|c|c|c|}
\hline Stratum & $\begin{array}{l}\text { Thickness, } \\
\mathrm{ft}\end{array}$ & $\begin{array}{l}\text { Depth, } \\
\text { ft }\end{array}$ \\
\hline \multicolumn{3}{|l|}{ Chouteau Formation: } \\
\hline Limestone, dolomite, gray, dense & 12 & 1,294 \\
\hline \multicolumn{3}{|l|}{ Devonian system: } \\
\hline $\begin{array}{l}\text { Limestone, gray white to brown, dense to } \\
\text { lithographic, sandy at base }\end{array}$ & 80 & 1,374 \\
\hline \multicolumn{3}{|l|}{$\begin{array}{l}\text { Ordovician system: } \\
\text { Black River (Decorah Formation?): }\end{array}$} \\
\hline $\begin{array}{l}\text { Limestone, gray-white, densely crystalline, } \\
\text { argillaceous }\end{array}$ & 29 & 1,403 \\
\hline \multicolumn{3}{|l|}{ St. Peter Formation: } \\
\hline Sandstone, white & 73 & 1,476 \\
\hline Shale, green & 13 & 1,489 \\
\hline \multicolumn{3}{|l|}{$\begin{array}{l}\text { Canadian (of E. O. Ulrich) system: } \\
\text { Jefferson City Formation: }\end{array}$} \\
\hline $\begin{array}{l}\text { Dolomite, gray-white to buff, cherty, } \\
\text { argillareous }\end{array}$ & 174 & 1,663 \\
\hline \multicolumn{3}{|l|}{ Roubidoux Formation: } \\
\hline $\begin{array}{l}\text { Dolomite, gray-white, cherty, sandy, distinct } \\
\text { sandstone at base }\end{array}$ & 147 & 1,810 \\
\hline \multicolumn{3}{|l|}{$\begin{array}{l}\text { Ozarkian (of E. O. Ulrich) system: } \\
\text { Gasconade Formation: }\end{array}$} \\
\hline $\begin{array}{l}\text { Dolomite, gray-white, cherty; base not } \\
\text { reached }\end{array}$ & 10 & 1,820 \\
\hline
\end{tabular}




\section{APPENDIX B}

\section{Well Water-Level Elevations}




\section{B-1}

$02 / 05 / 93$

KANSAS CITY DOE PLANT

WELL WATER LEVEL ELEVATIONS

WELL KC84-009-L

COORDINATES

North: $\quad 310051.30$

East : $\quad 843734.06$

MEASUREMENT PER 100

from : $12 / 10 / 84$

To: $07 / 14 / 92$

WATER LEVEL ELEVATION STRTISTICS

Mean : $\quad 792.95$

Std: $\quad 0.28$

Var : $\quad 0.08$

High: $\quad 793.36$

LOW: $\quad 792.23$

Range: $\quad 1.13$

Size: $\quad 40$

SORTED WATER LEVEL ELEVATIONS

$\begin{array}{llll}\text { RANK } & \text { ELEV } & \text { OTR } & \text { YEAR } \\ & & & \\ 1 & 793.36 & 1 & 85 \\ 2 & 793.36 & 1 & 85 \\ 3 & 793.30 & 2 & 85 \\ 4 & 793.30 & 2 & 85 \\ 5 & 793.30 & 2 & 85 \\ 6 & 793.30 & 2 & 85 \\ 7 & 793.30 & 3 & 85 \\ 8 & 793.30 & 3 & 85 \\ 9 & 793.30 & 3 & 85 \\ 10 & 793.13 & 4 & 86 \\ 11 & 793.09 & 4 & 86 \\ 12 & 793.09 & 1 & 87 \\ 13 & 793.09 & 2 & 87 \\ 14 & 793.07 & 2 & 86 \\ 15 & 793.03 & 4 & 86 \\ 16 & 793.03 & 3 & 90 \\ 17 & 793.01 & 4 & 85 \\ 18 & 793.00 & 4 & 85 \\ 19 & 793.00 & 3 & 87 \\ 20 & 792.99 & 1 & 91 \\ 21 & 792.94 & 3 & 91 \\ 22 & 792.92 & 3 & 85 \\ 23 & 792.91 & 2 & 86 \\ 24 & 792.88 & 2 & 86 \\ 25 & 792.88 & 3 & 87 \\ 26 & 792.86 & 4 & 85 \\ 27 & 792.86 & 3 & 86 \\ 28 & 792.82 & 1 & 86 \\ 29 & 792.82 & 3 & 86 \\ 30 & 792.80 & 4 & 85 \\ 31 & 792.80 & 1 & 86 \\ 32 & 792.75 & 1 & 85 \\ 33 & 792.73 & 3 & 92 \\ 34 & 792.64 & 1 & 91 \\ 35 & 792.59 & 1 & 86 \\ 36 & 792.59 & 1 & 85 \\ 37 & 792.55 & 1 & 85 \\ 38 & 792.55 & 2 & 87 \\ 39 & 792.40 & 4 & 84 \\ 40 & 792.23 & 4 & 89\end{array}$


02/05/93

KANSAS CITY DOE PLANT

WELL WATER LEVEL ELEVATIONS

WELL KC84-009-M

COORDINATES

North: $\quad 310051.30$

East : $\quad 843734.06$

MEASUREMENT PERIOD

from : $12 / 10 / 84$

To: $07 / 11 / 91$

WATER LEVEL ELEVATION STATISTICS

$\begin{array}{lr}\text { Mean : } & 792.88 \\ \text { Std : } & 0.18 \\ \text { Var : } & 0.03 \\ \text { High : } & 793.14 \\ \text { Low : } & 792.31 \\ \text { Range: } & 0.83 \\ \text { Size : } & 41\end{array}$

SORTED WATER LEVEL ELEVATIONS

$\begin{array}{cccc}\text { RANK } & \text { ELEV } & \text { OTR } & \text { YEAR } \\ 1 & 793.14 & 1 & 85 \\ 2 & 793.14 & 1 & 85 \\ 3 & 793.12 & 4 & 86 \\ 4 & 793.10 & 2 & 86 \\ 5 & 793.06 & 4 & 85 \\ 6 & 793.06 & 1 & 87 \\ 7 & 793.06 & 2 & 87 \\ 8 & 793.06 & 4 & 85 \\ 9 & 793.04 & 4 & 86 \\ 10 & 793.04 & 4 & 86 \\ 11 & 792.97 & 3 & 85 \\ 12 & 792.97 & 2 & 85 \\ 13 & 792.97 & 2 & 85 \\ 14 & 792.97 & 2 & 85 \\ 15 & 792.97 & 3 & 87 \\ 16 & 792.92 & 2 & 86 \\ 17 & 792.92 & 3 & 90 \\ 18 & 792.89 & 3 & 86 \\ 19 & 792.89 & 3 & 87 \\ 20 & 792.87 & 3 & 86 \\ 61 & 792.87 & 1 & 85 \\ 22 & 792.87 & 3 & 85 \\ 23 & 792.87 & 2 & 86 \\ 24 & 792.86 & 1 & 91 \\ 25 & 792.85 & 2 & 85 \\ 26 & 792.85 & 3 & 85 \\ 27 & 792.85 & 3 & 85 \\ 28 & 792.85 & 4 & 85 \\ 29 & 792.85 & 4 & 85 \\ 30 & 792.85 & 1 & 86 \\ 31 & 792.85 & 3 & 86 \\ 32 & 792.80 & 3 & 91 \\ 33 & 792.76 & 1 & 85 \\ 34 & 792.76 & 1 & 86 \\ 35 & 792.75 & 1 & 88 \\ 36 & 792.74 & 1 & 86 \\ 37 & 792.68 & 1 & 85 \\ 38 & 792.66 & 4 & 84 \\ 39 & 792.62 & 1 & 91 \\ 40 & 792.39 & 2 & 87 \\ 41 & 792.31 & 4 & 89\end{array}$


$02 / 05 / 93$

WELL KC84-009-U

COORDINATES

North: $\quad 310051.30$

East : $\quad 843734.06$

MEASUREMENT PERIOD

From : $12 / 10 / 84$

To : 07/13/92

WATER LEVEL ELEVATION STATISTICS

$\begin{array}{lr}\text { Mean : } & 792.87 \\ \text { Std : } & 0.18 \\ \text { Var : } & 0.03 \\ \text { High : } & 793.13 \\ \text { Low : } & 792.39 \\ \text { Range: } & 0.82 \\ \text { Size : } & 42\end{array}$

KANSAS CITY DOE PLANT

WELL WATER LEVEL ELEVATIONS

SORTED WATER LEVEL ELEVATIONS

$\begin{array}{cccc}\text { RANK } & \text { ELEJ } & \text { OTR } & \text { YEAR } \\ 1 & 793.13 & 1 & 85 \\ 2 & 793.13 & 1 & 85 \\ 3 & 793.09 & 4 & 85 \\ 4 & 793.09 & 2 & 86 \\ 5 & 793.09 & 4 & 86 \\ 6 & 793.09 & 4 & 85 \\ 7 & 793.07 & 4 & 86 \\ 8 & 793.04 & 4 & 86 \\ 9 & 793.00 & 2 & 87 \\ 10 & 793.00 & 3 & 85 \\ 11 & 792.98 & 1 & 87 \\ 12 & 792.96 & 2 & 85 \\ 13 & 792.96 & 2 & 85 \\ 14 & 792.95 & 2 & 85 \\ 15 & 792.96 & 3 & 85 \\ 16 & 792.96 & 3 & 86 \\ 17 & 792.96 & 3 & 87 \\ 18 & 792.93 & 3 & 90 \\ 19 & 792.92 & 3 & 85 \\ 20 & 792.92 & 3 & 86 \\ 21 & 792.90 & 1 & 85 \\ 22 & 792.90 & 3 & 85 \\ 23 & 792.90 & 2 & 86 \\ 24 & 792.87 & 1 & 91 \\ 25 & 792.86 & 2 & 86 \\ 26 & 792.86 & 3 & 87 \\ 27 & 792.84 & 2 & 85 \\ 28 & 792.82 & 4 & 85 \\ 29 & 792.82 & 3 & 91 \\ 30 & 792.79 & 1 & 85 \\ 31 & 792.79 & 1 & 86 \\ 32 & 792.77 & 3 & 86 \\ 33 & 792.76 & 1 & 88 \\ 34 & 792.75 & 4 & 85 \\ 35 & 792.75 & 1 & 86 \\ 36 & 792.73 & 1 & 86 \\ 37 & 792.72 & 3 & 92 \\ 38 & 792.71 & 4 & 84 \\ 39 & 792.67 & 1 & 85 \\ 40 & 792.58 & 1 & 91 \\ 41 & 792.32 & 2 & 87 \\ 42 & 792.31 & 4 & 89\end{array}$


WELL XC84-015

COORDINATES

North: $\quad 309563.74$

East : $\quad 844588.69$

MEASUREMENT PERIOD

From : $12 / 10 / 84$

To: 03/26/91

WATER LEVEL ELEVATION STATISTICS

$\begin{array}{lr}\text { Mesn : } & 765.23 \\ \text { Std : } & 1.31 \\ \text { Var : } & 1.72 \\ \text { High : } & 768.14 \\ \text { Low : } & 762.84 \\ \text { Range: } & 5.30 \\ \text { Size : } & 34\end{array}$

SORTED WATER LEVEL ELEVATIONS

$\begin{array}{cccc}\text { RANK } & \text { ELEV } & \text { OTR } & \text { YEAR } \\ 1 & 768.14 & 1 & 87 \\ 2 & 767.83 & 1 & 85 \\ 3 & 767.83 & 1 & 85 \\ 4 & 766.22 & 4 & 86 \\ 5 & 766.83 & 2 & 87 \\ 6 & 766.70 & 1 & 87 \\ 7 & 766.14 & 4 & 86 \\ 8 & 766.03 & 1 & 85 \\ 9 & 765.68 & 2 & 85 \\ 10 & 765.58 & 2 & 86 \\ 11 & 765.53 & 2 & 85 \\ 12 & 765.51 & 4 & 85 \\ 13 & 765.43 & 3 & 87 \\ 14 & 765.49 & 2 & 85 \\ 15 & 765.29 & 3 & 85 \\ 16 & 765.24 & 1 & 85 \\ 17 & 765.20 & 4 & 85 \\ 18 & 765.10 & 1 & 86 \\ 19 & 765.03 & 2 & 87 \\ 20 & 764.89 & 2 & 85 \\ 21 & 764.83 & 3 & 85 \\ 22 & 764.79 & 4 & 84 \\ 23 & 764.78 & 1 & 86 \\ 24 & 764.53 & 3 & 85 \\ 25 & 764.51 & 3 & 86 \\ 26 & 764.39 & 3 & 86 \\ 27 & 764.35 & 3 & 87 \\ 28 & 764.33 & 2 & 86 \\ 29 & 764.12 & 1 & 85 \\ 30 & 763.91 & 1 & 88 \\ 31 & 763.53 & 4 & 87 \\ 32 & 763.27 & 1 & 91 \\ 33 & 763.05 & 4 & 88 \\ 34 & 762.84 & 4 & 89\end{array}$


02/05/93

WELL KC84-016

COORDINATES

North: $\quad 309584.99$

East : $\quad 844667.94$

MEASUREMENT PER 100

From : $12 / 10 / 84$

To: $03 / 26 / 91$

WATER LEVEL ELEVATION STATISTICS

$\begin{array}{lr}\text { Mean : } & 763.75 \\ \text { Std : } & 1.88 \\ \text { Var : } & 3.55 \\ \text { High : } & 772.75 \\ \text { Low : } & 761.64 \\ \text { Range: } & 19.11 \\ \text { Size: } & 41\end{array}$

KANSAS CITY DOE PLANT

WELL WATER LEVEL ELEVATIONS

SORTED WATER LEVEL ELEVATIONS

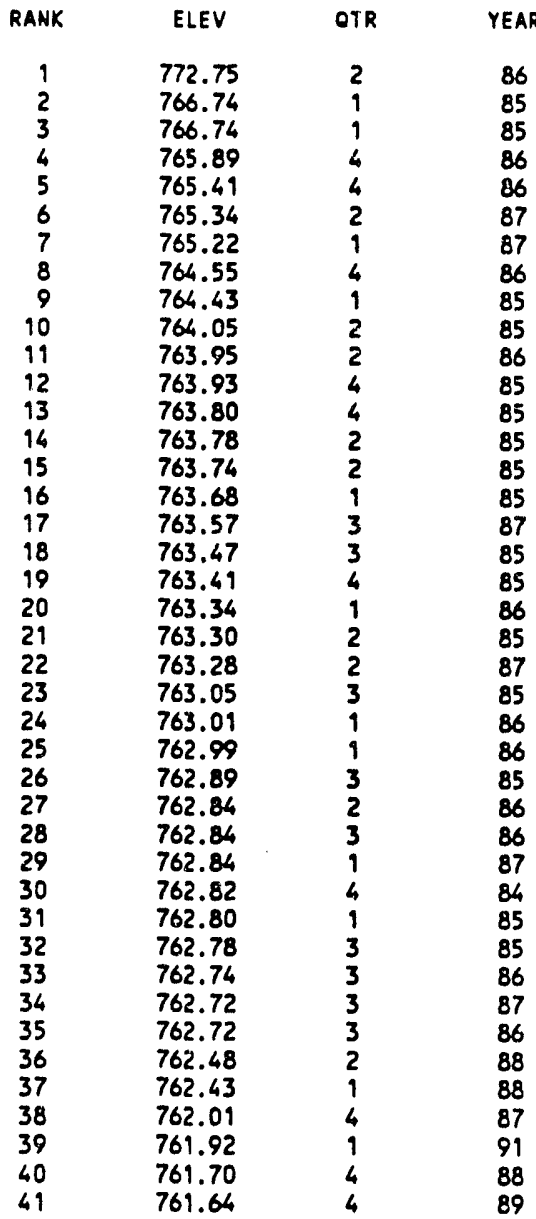


$02 / 05 / 93$

WELL KC84.017

COORDINATES

North: $\quad 309672.79$

East : $\quad 844696.00$

MEASUREMENT PERIOO

From : $12 / 10 / 84$

To: $03 / 26 / 91$

WATER LEVEL ELEVATION STATISTICS

$\begin{array}{lr}\text { Mean : } & 775.42 \\ \text { Std : } & 1.01 \\ \text { Var : } & 1.01 \\ \text { High : } & 776.99 \\ \text { Low : } & 773.23 \\ \text { Range: } & 3.76 \\ \text { Size: } & 42\end{array}$

KANSAS CITY DOE PLANT

WELL WATER LEVEL ELEVATIONS

SORTED WATER LEVEL ELEVATIONS

$\begin{array}{cccc}\text { RANK } & \text { ELEV } & \text { OIR } & \text { YEAR } \\ & & & \\ 1 & 776.99 & 4 & 85 \\ 2 & 776.99 & 4 & 85 \\ 3 & 776.94 & 4 & 86 \\ 4 & 776.94 & 2 & 87 \\ 5 & 776.82 & 4 & 86 \\ 6 & 776.76 & 1 & 85 \\ 7 & 776.76 & 1 & 85 \\ 8 & 776.40 & 4 & 85 \\ 9 & 776.32 & 3 & 87 \\ 10 & 776.24 & 4 & 85 \\ 11 & 776.15 & 1 & 87 \\ 12 & 776.13 & 2 & 87 \\ 13 & 776.11 & 1 & 85 \\ 14 & 776.07 & 2 & 85 \\ 15 & 775.88 & 1 & 85 \\ 16 & 775.88 & 4 & 86 \\ 17 & 775.69 & 1 & 86 \\ 18 & 775.63 & 4 & 84 \\ 19 & 775.59 & 2 & 85 \\ 20 & 775.47 & 2 & 85 \\ 21 & 775.46 & 1 & 87 \\ 22 & 775.34 & 2 & 85 \\ 23 & 775.32 & 3 & 87 \\ 24 & 775.17 & 1 & 85 \\ 25 & 775.13 & 3 & 85 \\ 26 & 775.05 & 3 & 85 \\ 27 & 775.05 & 1 & 86 \\ 28 & 775.01 & 2 & 88 \\ 29 & 774.90 & 1 & 88 \\ 30 & 774.80 & 3 & 85 \\ 31 & 774.76 & 3 & 85 \\ 32 & 774.65 & 1 & 86 \\ 33 & 774.63 & 2 & 86 \\ 34 & 774.56 & 2 & 86 \\ 35 & 774.49 & 2 & 86 \\ 36 & 774.34 & 3 & 86 \\ 37 & 774.28 & 3 & 86 \\ 38 & 774.24 & 3 & 86 \\ 39 & 774.08 & 4 & 87 \\ 40 & 773.85 & 4 & 89 \\ 41 & 773.42 & 1 & 91 \\ 42 & 773.23 & 4 & 88\end{array}$


$02 / 05 / 93$

WELL KC84.023

COORDINATES

North: $\quad 309694.49$

East : $\quad 844609.48$

MEASUREMENT PER 100

From : $12 / 10 / 84$

To: $08 / 04 / 87$

WATER LEVEL ELEVATION STATISTICS

$\begin{array}{lr}\text { Mean : } & 777.79 \\ \text { Std : } & 1.25 \\ \text { Var : } & 1.56 \\ \text { High : } & 778.28 \\ \text { Low : } & 771.41 \\ \text { Range: } & 6.87 \\ \text { Size : } & 29\end{array}$

KANSAS CITY DOE PLANT WELL WATER LEVEL ELEVATIONS

SORTED WATER LEVEL ELEVATIONS

$\begin{array}{cccc}\text { RANK } & \text { ELEV } & \text { OTR } & \text { YEAR } \\ 1 & 778.28 & 2 & 87 \\ 2 & 778.27 & 1 & 87 \\ 3 & 778.20 & 1 & 85 \\ 4 & 778.20 & 3 & 87 \\ 5 & 778.20 & 3 & 87 \\ 6 & 778.18 & 2 & 85 \\ 7 & 778.16 & 2 & 85 \\ 8 & 778.16 & 1 & 86 \\ 9 & 778.16 & 2 & 86 \\ 10 & 778.12 & 1 & 85 \\ 11 & 778.12 & 2 & 85 \\ 12 & 778.12 & 2 & 86 \\ 13 & 778.12 & 3 & 86 \\ 14 & 778.12 & 4 & 86 \\ 15 & 778.10 & 4 & 85 \\ 16 & 778.05 & 1 & 85 \\ 17 & 778.05 & 3 & 85 \\ 18 & 778.05 & 1 & 85 \\ 19 & 778.01 & 1 & 85 \\ 20 & 778.01 & 3 & 86 \\ 21 & 778.01 & 4 & 86 \\ 22 & 777.99 & 2 & 87 \\ 23 & 777.85 & 4 & 85 \\ 24 & 777.78 & 3 & 85 \\ 25 & 777.74 & 1 & 87 \\ 26 & 777.49 & 4 & 84 \\ 27 & 777.45 & 3 & 85 \\ 28 & 777.41 & 1 & 86 \\ 29 & 771.41 & 2 & 85\end{array}$


$02 / 05 / 93$

WELL KC85-042

COORDINATES

North: $\quad 309556.07$

East : $\quad 864708.91$

MEASUREMENT PERIOO

From : $06 / 25 / 85$

io : 07/26/92

WATER LEVEL ELEVATION STATISTICS

$\begin{array}{lr}\text { Mean : } & 759.76 \\ \text { Std : } & 0.91 \\ \text { Var : } & 0.83 \\ \text { High : } & 763.11 \\ \text { Low : } & 758.49 \\ \text { Range: } & 4.62 \\ \text { Size: } & 35\end{array}$

$\begin{array}{cccc}\text { RANK } & \text { ELEV } & \text { OTR } & \text { YEAR } \\ 1 & 763.11 & 2 & 87 \\ 2 & 760.95 & 4 & 86 \\ 3 & 760.78 & 4 & 85 \\ 4 & 760.78 & 4 & 85 \\ 5 & 760.70 & 1 & 87 \\ 6 & 760.65 & 2 & 86 \\ 7 & 760.63 & 4 & 86 \\ 8 & 760.61 & 4 & 86 \\ 9 & 760.28 & 2 & 85 \\ 10 & 760.28 & 3 & 85 \\ 11 & 760.11 & 3 & 85 \\ 12 & 760.11 & 4 & 85 \\ 13 & 759.95 & 4 & 85 \\ 14 & 759.74 & 3 & 86 \\ 15 & 759.65 & 3 & 86 \\ 16 & 759.61 & 3 & 87 \\ 17 & 759.53 & 1 & 86 \\ 18 & 759.51 & 3 & 85 \\ 19 & 759.50 & 2 & 86 \\ 20 & 759.49 & 3 & 85 \\ 21 & 759.49 & 1 & 86 \\ 22 & 759.43 & 2 & 86 \\ 23 & 759.43 & 2 & 87 \\ 24 & 759.41 & 3 & 86 \\ 25 & 759.30 & 1 & 86 \\ 26 & 759.28 & 3 & 87 \\ 27 & 759.28 & 1 & 87 \\ 28 & 758.97 & 2 & 88 \\ 29 & 758.95 & 1 & 88 \\ 30 & 758.88 & 3 & 88 \\ 31 & 758.85 & 4 & 87 \\ 32 & 758.78 & 4 & 88 \\ 33 & 758.72 & 3 & 92 \\ 34 & 758.54 & 1 & 91 \\ 35 & 758.49 & 4 & 89\end{array}$

KANSAS CITY DOE PLANT WELL WATER LEVEL ELEVATIONS 
$02 / 05 / 93$

WELL KC89-123

COORDINATES

North: $\quad 310066.02$

Eost : $\quad 843716.17$

MEASUREMENT PERIOO

From : $12 / 16 / 89$

To: $10 / 09 / 92$

WATER LEVEL ELEVATION STATISTICS

$\begin{array}{lr}\text { Mean : } & 789.17 \\ \text { Std : } & 3.41 \\ \text { Var : } & 11.61 \\ \text { High : } & 792.32 \\ \text { Low : } & 780.42 \\ \text { Range: } & 11.90 \\ \text { Size : } & 13\end{array}$

SORTED WATER LEVEL ELEVATIONS

$\begin{array}{cccc}\text { RANK } & \text { ELEV } & \text { OTR } & \text { YEAR } \\ 1 & 792.32 & 2 & 92 \\ 2 & 792.24 & 1 & 92 \\ 3 & 792.02 & 4 & 92 \\ 4 & 790.82 & 2 & 91 \\ 5 & 790.76 & 1 & 91 \\ 6 & 790.53 & 4 & 91 \\ 7 & 790.24 & 1 & 90 \\ 8 & 789.95 & 4 & 89 \\ 9 & 789.36 & 3 & 91 \\ 10 & 789.06 & 3 & 90 \\ 11 & 786.95 & 1 & 91 \\ 12 & 786.54 & 4 & 90 \\ 13 & 780.42 & 2 & 90\end{array}$

KanSAS CJTY DOE PLANT WELL WATER LEVEL ELEVATIONS 


\section{APPENDIX C}

Analytical Data from Selected Wells 


\section{APPENDIX C \\ Analytical Data from Selected Wells}

\section{Explanations:}

- All concentrations are micrograms per liter $(\mu \mathrm{g} / \mathrm{L})$.

- $\quad \mathrm{NA}=$ not analyzed

- $\quad \mathrm{U}=$ compound was analyzed for but not dectected.

The number is the dection limit for the sample. 
KANSAS CIIY : SELECTEO ANALYSIS FROM KEQ5.033.L

Constituent

us/L

$05 / 13 / 85$

$07 / 28 / 85$

$10 / 27 / 85$

Sample Date

$01 / 31 / 86$

C5/07/86

$09 / 04 / 86$

$10 / 27 / 85$

$6210 \div 187$

$1,1,1,2$ - TE TRACHLOROE THANE

$1,1,1$ - Trichloroet thane

$1,1,2,2$ - TE TRACHLOROETHANE

$1,1,2$ - TRICHLOROE THANE

1,1,2- TRICKLOROTRIFLLOROETH

1,1 -OICHLCRDE THANE

1,1 -OICKLOROETHENE

1,1 -OICHLOROPROPENE

$1,2,3$-TRICHLOROBEN2ENE

$1,2,3$ - TRICHLOROPROPANE

$1,2,3$ - TRIMETHYLBENZENE

$1,2,4$ - TR!CHLOROBENZENE

1,2-01BROMO-3-CHLOROPROPANE

1,2-01 aromoe thane

1,2-0ICHLOROBENZENE

1,2-O1CHLOROE THANE

1,2-OICHLOROETHENE (total)

1,2-01CHLOROPROPANE

$1,3,5$ - TRIMETHYLBENZENE

1,3-DICHLOROBEMZENE

1,3-DICHLOROPROPANE

1,6-DICHLOROBEMZENE

2,2-01CHLOROPRDPANE

2. BUTANOWE

2- CHLOROETHYLVINYL ETKER

2- CHLOROTOLUENE

2- HEXANOWE

4- CHLOROTOLUEME

6-METHYL-2-PENTANONE

ACETOWE

ACROLEIN

ACRYLONITRILE

BENZENE

BROMOBENZENE

BROMOCHLORCME THANE

BROMOFORM

CARBON DISULF SDE

CARBOW TETRACHLORIDE

CHLOROBE MZENE

CHLORDO I BROMOMETHANE

CHLOROE THANE

CHLORDETHENE

CHLOROFORM

CIS-1,2-01CHLOROETHENE

CIS-1,3-OICHLOROPROPENE

O! BROMOME THANE

Dichlor OBromome thame

DICHLORDOIFLUOROME THANE

ETMYLBENZENE
NA

NA

U

NA

U

NA

$U$
$U$
$U$

NA MA

4.8
2.84

2.8

MA

9.2

MA

NA

MA

MA

NA

Na

NA

MA

NA

MA

$u$
$u$
$u$

MA

MA

MA

$u$

U

u

$60.3^{\mathrm{N}}$

NA

NA

NA

NA

$U$

31.4

HA

NA

MA

NA

NA

MA

73.7

NA

NA

NA

MA

NA

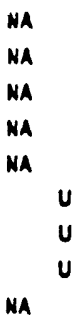

NA

$U$

MA

11.6 HI

$\mathrm{NA}$

NA

NA

NA

NA

MA

NA

NA

NA

23.4

\section{NA}

MA

MA

MA

NA

WA

U

MA

NA

MA

NA

NA

u

U

MA

HA

NA

$U$
$U$
$U$
$U$
42.5
$U$

MA

NA

$u$

$$
\begin{aligned}
& u \\
& U
\end{aligned}
$$

NA

8.6

6.5

MA

NA

NA

HA

MA

NA

NA

NA

MA U U
$21.0^{\circ}$

MA

MA

MA

NA

MA

MA

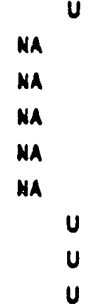

MA

NA

NA

in

u

$83.7^{U}$

U

in

MA $u$

NA

MA

NA

MA

KA

NA

NA

HA

NA

MA

NA

NA

NA

NA

NA

NA

NA

NA

$U$

u

NA

NA

NA

91.2

in

NA $U$

$$
\text { u }
$$$$
v
$$

Nid

$u$

$30.0^{U}$

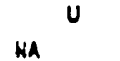

$u$
$u$
$u$

MA

NA
RA
NA
NA
NA
NA
NA
HA

32.1

NA

NA

NA

NA

NA

in

NA
$N A$
$N A$
$N A$
$N A$

NA
NA

NA

NA

$u$
$u$

75.3

NA
NA U

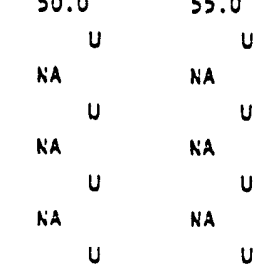

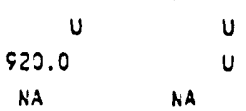

NA NA

NA NA

NA

NA

NA

NA

NA

$$
\begin{aligned}
& u \\
& u \\
& u
\end{aligned}
$$

NA

NA

NA

NA

NA

U

NA

${ }_{\text {NA }}^{\text {NA }}$

U

$\begin{array}{lllll} & U & & & \\ \text { NA } & & \text { NA } & \\ \text { NA } & & \text { HA } & \\ \text { NA } & & \text { NA } & \\ & & & & \\ & & & & \\ & & & \end{array}$

NA NA

NA NA

NA

u

\begin{tabular}{|c|c|}
\hline$U$ & $U$ \\
\hline$U$ & $u$ \\
\hline$u$ & $u$ \\
\hline
\end{tabular}

u

U

5.0

NA. 
Constituent

$\mathrm{wo/L}$

\begin{abstract}
KANSAS CITY : SELECTED ANALYSIS FROM KCB5.033.L
Semple Date

Q6/13/25

$07 / 28 / 85$

$10 / 27 / 85$

$09 / 31 / 86$

$05 / 07 / 86 \quad .08 / 04 / 86$

$10 / 27 / 86$

$02 / 06 / 87$
\end{abstract}

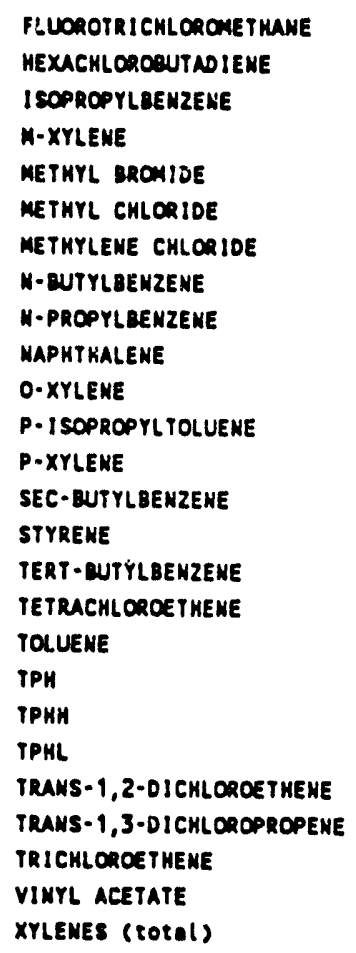

$M A$
$M A$
$M A$



2.8
$M A$
$M A$
$M A$
$M A$
$M A$
$M A$
$M A$
$M A$
$M A$


$M A$

$\sum_{m a} u$

MA

MA

MA

WA

wa

MA

MA

MA U

$U$
$U$

NA

NA

MA

ma

MA

$655.0^{U}$

MA

MA

$343.0^{U}$

Ma

MA

MA

$M_{M A} U$

$\begin{array}{lll} & U \\ \text { MA } & \\ \text { NA } & \\ & & \\ & U \\ & U \\ & U \\ & & \\ \text { NA } & & \end{array}$

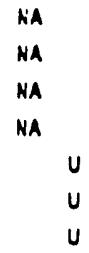

NA

NA

NA

NA

U

WA NA HA NA

MA HA NA NA NA

MA HA NA NA NA

MA

MA

NA

MA

MA

u

$u$

MA HA

MA NA

MA

NA

$420.0^{U}$

in

MA

MA

NA

HA

WA

NA

NA

NA

HA

NA

NA

HA

NA

NA

NA

NA $U$

A

\begin{tabular}{|c|c|}
\hline NA & NA \\
\hline NA & N \\
\hline NA & NA \\
\hline NA & NA \\
\hline u & $u$ \\
\hline 260.0 & 180.0 \\
\hline u & U \\
\hline & $u$ \\
\hline
\end{tabular}

MA 
KANSLS CITY : SELECTED RMALYSIS FROM KCE5.033.L

Constituent

ug/L

05/09/87

Sample Date

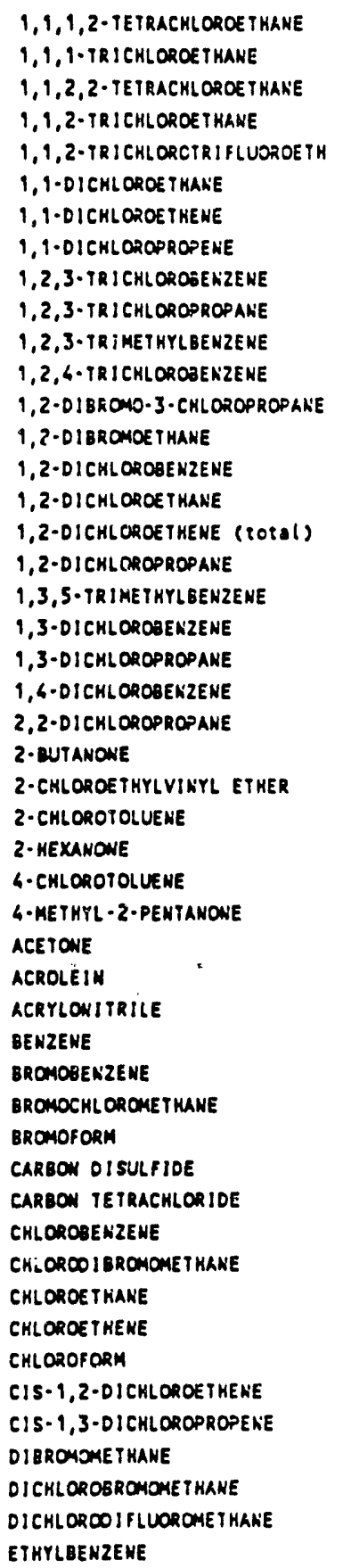

\begin{tabular}{|c|c|c|c|c|c|c|c|}
\hline Wh & MA & NA & NA & NA & HA & $N^{\prime} A$ & NA \\
\hline$u$ & 1.00 & 1.00 & 1.00 & $2.0 \mathrm{~s}$ & $1.0 \mathrm{U}$ & $1.0 \mathrm{~s}$ & $5.0 \mathrm{~J}$ \\
\hline$u$ & 1.00 & 1.00 & 1.00 & 2.04 & i.os & 1.00 & 5.00 \\
\hline$u$ & $1.0 \mathrm{U}$ & 1.00 & 1.00 & $2.0 \mathrm{~J}$ & $1.0 \mathrm{~J}$ & $1.0 \mathrm{~J}$ & $5.0 \mathrm{~J}$ \\
\hline NA & NA & HA & NA & NA & $N^{\prime} A$ & NA & $N^{\prime \prime} A$ \\
\hline 3.0 & 1.00 & 3.0 & 1.0 & 2.00 & 1.0 & $1.0 \mathrm{~J}$ & 5.00 \\
\hline 1.0 & 2.0 & 1.0 & $1.0 \mathrm{~s}$ & 2.00 & $1.0 \mathrm{~J}$ & 1.0. & 5.00 \\
\hline NA & NA & NA & HiA & NA & NA & HA & $N A$ \\
\hline NA & NA & NA & NA & HA & NA & $A A$ & $\mathrm{KA}$ \\
\hline NA & NA & NA & NA & NA & NA & $N A$ & Nh \\
\hline$n i$ & $N A$ & NA & NA & NA & NA & NA & BA \\
\hline Nin & WA & NA & HA & HA & HA & NA & NA \\
\hline WA & NA & NA & NA & NA & H'A & KA & i.A \\
\hline in & HA & NA & NA & NA & NA & N'A & NAA \\
\hline WA & HA & NA & MA & NA & NiA & NA & NA \\
\hline$u$ & 1.00 & 1.00 & 1.00 & 2.00 & 1.00 & $1.0 \mathrm{~J}$ & 5.00 \\
\hline 37.0 & 56.0 & 57.0 & 44.0 & 34.0 & 21.0 & $i 8.0$ & 15.0 \\
\hline $\mathbf{u}$ & 1.00 & 1.00 & 1.00 & $2.0 \mathrm{~J}$ & $9.0 \mathrm{U}$ & $1.0 \mathrm{~J}$ & $5.0 \mathrm{~s}$ \\
\hline MA & NA & NA & NA & NA & NA & NA & NA \\
\hline NA & NA & NA & NAA & HA & NA & NA & NA \\
\hline MA & WA & NA & NA & NA & NA & NA & NA \\
\hline NA & NA & MA & NA & NA & NA & $N A$ & NA \\
\hline MA & NA & NA & MA & NA & NA & $N A$ & $N A$ \\
\hline$u$ & 1.00 & 1.00 & 1.00 & 2.00 & 1.00 & 1.00 & 10.00 \\
\hline$u$ & $1.0 \mathrm{~s}$ & 1.00 & 1.00 & $2.0 \mathrm{~s}$ & $1.0 \mathrm{~s}$ & $1.0 \mathrm{~J}$ & $10.0 \mathrm{~J}$ \\
\hline MA & NA & NA & NA & NA & NA & NA & NA \\
\hline$u$ & 1.00 & 1.00 & $1.0 \mathrm{~J}$ & 2.00 & $1.0 U$ & 1.00 & 10.00 \\
\hline NA & MA & NA & HA & NA & NA & KA & Sil \\
\hline$u$ & 1.00 & 1.00 & $1.0 \mathrm{~J}$ & 2.00 & 1.00 & 1.00 & 10.00 \\
\hline NA & HA & NA & WA & HA & NA & SA & 10.00 \\
\hline WA & NA & HA & NA & HA & NA & $N A$ & 100.00 \\
\hline ind & HA & NA & HA & N2 & NA & NA & 100.00 \\
\hline $\mathbf{u}$ & 1.00 & 1.00 & $1.0 \mathrm{v}$ & 2.00 & 1.00 & $1.0 \mathrm{U}$ & $5.0 \mathrm{U}$ \\
\hline in & HA & NA & NA & NA & HA & NA & NA \\
\hline NA & NA & WA & NA & NA & NA & NA & NA \\
\hline $\boldsymbol{U}$ & 1.00 & 1.00 & 1.00 & 2.00 & $1.0 \mathrm{U}$ & 1.00 & 5.00 \\
\hline$u$ & 1.00 & 1.00 & 1.00 & 2.00 & 1.00 & $1.0 \mathrm{~J}$ & 5.0 .0 \\
\hline$u$ & 1.00 & 1.00 & 1.00 & 2.00 & $1.0 \mathrm{~s}$ & $1.0 \mathrm{u}$ & 5.00 \\
\hline$u$ & 1.00 & 1.00 & 1.00 & 2.05 & 1.0 .5 & 1.00 & $5.0 \mathrm{~J}$ \\
\hline$u$ & 1.00 & 1.00 & 1.00 & 2.00 & 1.00 & $1.0 \mathrm{~J}$ & 5.00 \\
\hline U & 1.00 & 1.00 & $1.0 \mathrm{~J}$ & $2.00 \mathrm{~J}$ & 1.01 & 1.00 & $10.0 \mathrm{~J}$ \\
\hline$i 6.0$ & 58.0 & 36.0 & 60.0 & 34.0 & 39.0 & 36.0 & 22.0 \\
\hline u & 1.00 & 1.00 & $1.0 \mathrm{~J}$ & $2.0 \mathrm{~J}$ & 1.001 & $1.00 \mathrm{~s}$ & 5.20 \\
\hline NA & in & SA & KA & NA & MA & NA & NA \\
\hline$u$ & נ. & 1.00 & $1.0 \mathrm{~J}$ & 2.00 & 1.00 & 1.00 & 5.2. \\
\hline NA & NA & NA & NA & NA & NA & NA & NA \\
\hline$u$ & 1.00 & 1.00 & 1.00 & $2.0 \mathrm{~J}$ & 1.00 & $1.0 \mathrm{~J}$ & $5.0 \mathrm{~J}$ \\
\hline NA & Wh & NA & NA & NA & $N A$ & $N A$ & NA \\
\hline$u$ & 1.00 & 1.00 & $1.0 \mathrm{U}$ & $2.0 \mathrm{~J}$ & $1.0 \mathrm{U}$ & $1.0 \mathrm{~J}$ & $5.0 \mathrm{~J}$ \\
\hline
\end{tabular}




$$
\text { C-4 }
$$

KANSAS CITY : SELECTED AKALYSIS FROY KC85-033-L

Const it tuent

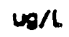

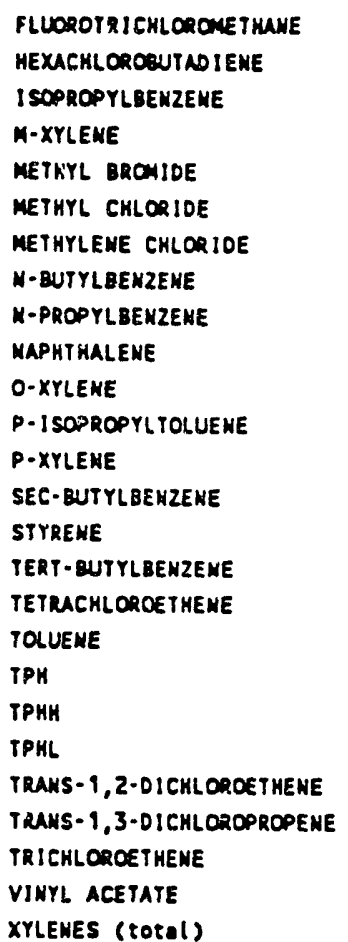

Semple Date

$\begin{array}{llllllll}05 / 01 / 87 & 08 / 01 / 87 & 10 / 29 / 87 & 01 / 31 / 88 & 06 / 28 / 88 & 08 / 01 / 88 & 10 / 31 / 88 & 02 / 15 / 89\end{array}$

\begin{tabular}{|c|c|c|c|c|c|c|c|}
\hline$m$ & $m$ & MA & $\boldsymbol{M}$ & $m$ & MA & NA & 10.04 \\
\hline$m$ & $M$ & MA & $m$ & $m$ & NA & NA & NA \\
\hline$m$ & แn & MA & $M$ & $M A$ & NA & NA & KA \\
\hline$m$ & $M$ & $\mathrm{kA}$ & $M$ & MA & NA & MA & NA \\
\hline$u$ & $1 . \boldsymbol{\alpha}$ & १.ou & $1 . \boldsymbol{\alpha}$ & 2.00 & 1.04 & 1.04 & 10.04 \\
\hline$u$ & 1.00 & 1.0 & 1.0 & 2.00 & 1.04 & 1.00 & 10.00 \\
\hline U & 1.0 & 3.0 & 1.00 & $2.0 \mathrm{~s}$ & 1.00 & 1.00 & 5.04 \\
\hline $\mathrm{HA}$ & $m$ & $m$ & $\mathrm{ma}$ & HA & KA & NA & NA \\
\hline MA & $M A$ & Ma & $M A$ & MA & WA & NA & NA \\
\hline Mh & NA & $\mathrm{HA}$ & MA & NA & HA & NA & NA \\
\hline MA & MA & WA & $\mathrm{MA}$ & NA & NA & NA & NA \\
\hline MA & NA & WA & NA & NA & NA & NA & HA \\
\hline$m$ & KA & MA & KA & KA & NA & NA & NA \\
\hline MA & KA & MA & MA & MA & NA & NA & NA \\
\hline$u$ & 1.00 & 1.00 & 1.04 & 2.00 & 1.00 & 1.00 & 5.00 \\
\hline$M A$ & $M$ & $M$ & KA & NA & HA & KA & NA \\
\hline$u$ & 1.00 & 1.00 & 1.00 & 2.00 & 1.00 & 1.00 & 5.00 \\
\hline$u$ & 1.00 & 1.00 & 1.00 & 2.00 & 1.00 & 1.00 & 5.00 \\
\hline MA & MA & MA & KA & MA & MA & NA & NA \\
\hline $\mathrm{MA}$ & $M A$ & MA & MA & NA & NA & NA & N \\
\hline$M A$ & MA & $M A$ & $M A$ & MA & NA & NA & NA \\
\hline MA & MA & WA & MA & MA & KA & NA & NA \\
\hline U & 1.00 & 1.00 & $1 . \infty$ & 2.00 & 1.00 & 1.00 & 5.00 \\
\hline 160.0 & 71.0 & 3.0 & 250.0 & 260.0 & 120.0 & 100.0 & 79.0 \\
\hline u & 1.00 & 1.00 & 1.00 & 2.00 & 1.00 & 9.00 & 10.04 \\
\hline$u$ & 1.00 & 1.00 & 1.01 & 2.04 & 1.00 & 1.04 & $5.0 \mathrm{y}$ \\
\hline
\end{tabular}


KANSAS GITY : SELECTED ARALLYSIS FROM KCE5.033.L

Constituent

us/L
05/01/89

$07 / 28 / 89$
Sample Date

$02 / 04 / 90 \quad 04 / 28 / 90 \quad[7 / 2 t / 50 \quad 12 / 12 / 50$

$(i / 1 i / 91$

\begin{tabular}{|c|c|c|c|c|c|c|c|c|}
\hline 1,1,1,2-TETRACHLOROETHAHE & NA & NA & NA & $4.0 \mathrm{~J}$ & $4.0 \mathrm{U}$ & 4.00 & K'A & AA \\
\hline 1,1,9-TRICHLORCETHAGE & 5.0 .4 & 5.00 & $5.0 \mathrm{~s}$ & 4.04 & 4.00 & $4.0 \mathrm{ds}$ & 5.04 & $5.0 \mathrm{~J}$ \\
\hline 1,1,2,2-TETRACHLOFOETHAHE & 5.00 & $5.0 \mathrm{U}$ & 5.01 & $4.0 \mathrm{~J}$ & $4.0 \mathrm{U}$ & 4.00 & $5.2 \mathrm{~J}$ & 5.0 .1 \\
\hline 1,1,2-TRICHLOROETHANE & $5.0 \mathrm{~s}$ & $5.0 \mathrm{~J}$ & 5.00 & $4.0 \mathrm{~J}$ & $4.0 \mathrm{~J}$ & $4.0 \mathrm{~N}$ & $5.0^{\prime \prime}$ & $5.0 \mathrm{~s}$ \\
\hline 1,1,2-TRICHLOROTRIFLUOROETH & in & hiA & NA & NiA & N'A & N'A & 5.00 & $5.0 \mathrm{~J}$ \\
\hline 1,1-DICHLOROETHANE & $5.0 \mathrm{~s}$ & 5.04 & $5.0 \mathrm{~J}$ & 4.018 & $4.0 \mathrm{U}$ & 4.00 & 5.815 & 8.0 \\
\hline 1,1-DICKLOROETHENE & $5.0 \mathrm{~s}$ & $5 . \alpha$ & 5.00 & 7.04 & $7.0 \mathrm{~s}$ & $7.0 \mathrm{~J}$ & $5.0 \mathrm{~J}$ & $5.0 \mathrm{~J}$ \\
\hline 1,1-OICHLOAOPROPEKE & in & NA & NA & $4.0 \mathrm{~J}$ & $4.0 \mathrm{~J}$ & $4.0 . \mathrm{J}$ & NA & NA \\
\hline 1,2,3-TRICHLOROSEKZEEHE & in & NA & in & 5.04 & $5.0 \mathrm{~J}$ & $5.0 \mathrm{~J}$ & k'A & NA \\
\hline 1,2,3-TRICHLOROPROPANE & kia & in & ha & $4.0 \mathrm{~J}$ & 4.00 & $4.0 \mathrm{~s}$ & N'A & NA \\
\hline $1,2,3$-TRIMETHYLEENZENE & MA & kA & in & $5.0 \mathrm{~J}$ & $5.0 . \mathrm{J}$ & $5.0 \mathrm{~J}$ & NA & AA \\
\hline 1,2,4-TRICHLOROSERZENE & in & NA & in & $5.0 \mathrm{~s}$ & $5.0 \mathrm{~s}$ & 5.0 .5 & NA & NA \\
\hline 1,2-DIGROMO-3-CHLOROPROPAKE & HA & in & NA & $4.0 \mathrm{~s}$ & $4.0 \mathrm{~s}$ & $4.0 \mathrm{~J}$ & iA & NA \\
\hline 1,2-DIGROMOETHKAE & ma & HA & in & $4.0 \mathrm{~s}$ & $4.0 \mathrm{~J}$ & $4.0 \mathrm{U}$ & IA & NA \\
\hline 1,2-DICHLOROBERIZENE & NA & NA & KA & 4.00 & $4.0 \mathrm{~J}$ & 4.00 & NA & NA \\
\hline 1,2-01CHLOROE THANE & 5.00 & 5.00 & 5.00 & $4.0 \mathrm{~J}$ & 4.00 & 4.00 & $5.0 \mathrm{~s}$ & 5.00 \\
\hline 1,2-DICHLOROETHENE (tOTAl) & 10.0 & 8.0 & 8.0 & WA & NA & in & 84.0 & 60.0 \\
\hline 1,2-DICHLOROPROPANE & 5.00 & 5.00 & 5.04 & 4.00 & 4.00 & 4.00 & 5.00 & 5.00 \\
\hline 1,3,5-TRIMETHYLBER'ZENE & Na & NA & NR & $5.0 \mathrm{U}$ & $5.0 \mathrm{U}$ & 5.00 & NA & NA \\
\hline 1,3-DICHLOROBELIZENE & NA & NA & kA & 4.00 & $4.0 \mathrm{~J}$ & 4.00 & NA & NA \\
\hline 1,3-DICHLOROPROPANE & kA & kiA & MA & $4.0 \mathrm{~s}$ & 4.00 & 4.00 & I:A & liA \\
\hline 1,4-DIC.HLOROBENZENE & MA & NA & NA & 4.00 & 4.00 & $4.0 \mathrm{~J}$ & NA & NA \\
\hline 2,2-DICHLOROPROPANE & NA & NA & NA & 4.0'J & 4.00 & 4.00 & liA & NA \\
\hline 2-BUTANONE & 10.00 & 10.00 & 10.00 & HA & N'A & NA & 10.00 & 5.00 \\
\hline 2-CHLOROETHYLVINYL ETHER & 90.00 & $10.0 \mathrm{~J}$ & NA & NA & NA & KA & NA & NA \\
\hline 2-CHLOROTOLUENE & NA & HA & NA & 4.00 & $4.0 \mathrm{~N}$ & 4.00 & NA & NA \\
\hline 2-KEXANOWE & 10.00 & 10.00 & $10.0 s$ & NA & HA & hiA & 5.00 & 5.00 \\
\hline 4-CHLOROTOLUENE & NA & NA & NA & $4.0 \mathrm{u}$ & $4.0 \mathrm{~J}$ & 4.00 & NA & NA \\
\hline 4-METHYL-2-PENTANOHE & 10.00 & 10.00 & 10.00 & NA & NA & NA & 5.00 & $5.0 \mathrm{~J}$ \\
\hline ACETONE & 10.00 &.$\quad 10.04$ & $10.0 \mathrm{~s}$ & HiA & NA & NA & $10.0 \mathrm{~J}$ & 10.04 \\
\hline ACROLEIN & 100.00 & - $\quad 100.00$ & NA & NA & NA & NA & NA & NA \\
\hline ACRYLONITRILE & $100.0 \mathrm{~V}$ & $=100.00$ & HA & NA & NA & NA & NA & NA \\
\hline BENZENE & 5.00 & $=5.04$ & 5.005 & 4.00 & $4.0 \mathrm{~s}$ & 4.00 & $5.0 \mathrm{~J}$ & $5.0 \mathrm{U}$ \\
\hline BROMOBENZENE & NA & NA & MA & 4.04 & $4.0 \mathrm{~J}$ & 4.00 & NA & NA \\
\hline BROMOCHLORONE THRKE & HA & HA & MA & 4.04 & 4.04 & 4.00 & NA & NA \\
\hline BROMOFORM & $5.0 \mathrm{~s}$ & 5.00 & 5.00 & $4.0 \mathrm{~J}$ & 4.00 & 4.00 & $5.0 \mathrm{~J}$ & 5.00 \\
\hline CARBOW DISULFIDE & $5.0 \mathrm{~J}$ & 5.00 & 5.00 & NA & HA & KA & NA & B:A \\
\hline CARBOW TETRACHLORIDE & 5.00 & 5.01 & 5.04 & 4.00 & $4.0 \mathrm{~s}$ & $4.0 \mathrm{~J}$ & $5.0 \mathrm{~s}$ & 5.0 .5 \\
\hline CHLOROBENZENE & $5.0 \mathrm{~s}$ & 5.00 & 5.00 & $4.0 \mathrm{~J}$ & 4.0 .5 & 4.00 & $5.0 \mathrm{~J}$ & 5.04 \\
\hline CHLOROO I BROMOMET TAKE & $5.0 \mathrm{~J}$ & 5.00 & 5.00 & 4.04 & 4.00 & 4.04 & $5.0 \mathrm{~J}$ & 5.04 \\
\hline CHLOROET THRE & $10.0 \mathrm{~J}$ & 10.00 & 10.00 & 4.00 & 4.00 & $4.0 \mathrm{~J}$ & 10.00 & 10.0 .1 \\
\hline CHLOROETHENE & 11.0 & 10.00 & 10.0 & 15.0 & 4.00 & 10.0 & 35.0 & $10.0 \mathrm{~J}$ \\
\hline CHLOROFORM & $5.0 \mathrm{~J}$ & $5.0 \mathrm{~J}$ & 5.00 & $4.0 \mathrm{~J}$ & 4.00 & $4.0 \mathrm{~J}$ & 5.00 & $5.0 \mathrm{~J}$ \\
\hline CIS-1,2-DICHLOROETHENE & NA & na & his & 9.0 & 10.0 & 7.0 & $\mathrm{NA}$ & NA \\
\hline CIS-1,3-DICHLOROPROPENE & $5.0 \mathrm{~J}$ & 5.00 & 5.00 & NiA & NA & NA & $5.01 \mathrm{~J}$ & $5.2 \mathrm{~J}$ \\
\hline DISROMOMETHANE & NA & HA & in & $4.0 \mathrm{~J}$ & $4.0 \mathrm{~J}$ & $4.0 \mathrm{~J}$ & NA & i.A \\
\hline DICHLOROSROMOME THLHE & $5.0 \mathrm{~J}$ & $5.0 \mathrm{U}$ & 5.095 & $4.0 \mathrm{~s}$ & 4.00 & 4.00 & 5.00 & $5.0 \mathrm{~s}$ \\
\hline DICHLORCO I FLUOROMET THRLE & NA & nA & NA & 8.04 & $8.0 \mathrm{~J}$ & $8.0 \mathrm{~J}$ & i:A & liA \\
\hline ETHYLBENZENE & 5.00 & 5.00 & 5.00 & $4.0 \mathrm{~J}$ & $4.0 \mathrm{~J}$ & $4.0 \mathrm{~s}$ & 5.00 & 5.01 \\
\hline
\end{tabular}




\section{C-6}

KANSAS CITY : SELECTED ANALYSIS FROM KC85.033.L

Constituent

ug/h

FLLOROTRICHLOROMETHAME MEXACKLORCQUTANDIEME ISOPROPYLBENZENE

M-XYLENE

METHYL CRONIDE

METHYL CHLCRIDE METHYLENE CHLORIDE

$N$-BUTYLBENZENE

W-PROPYLBENZENE

MAPHTHALEME

O.XYLENE

P. I SOPROPYL TOLUENE

P-XYLENE

SEC-BUTYLBENZENE

STYREME

TERT-EUTYLBENZENE

TETRACHLOROETHENE

TOLIJEME

TPH

TPHH

TPHL

TRANS-1,2-DICHLCROETHENE

TRANS-1,3-DICHLOROPROPEHE

TRICHLOROE THEME

VIWYL ACETATE

XYLENES (total)
05/01/89 07/28/89

$10 / 26 / 89$

Semple Date

$02 / 04 / 90 \quad 04 / 28 / 90 \quad 07 / 26 / 90 \quad 10 / 10 / 90 \quad 01 / 11 / 91$

\begin{tabular}{|c|c|c|c|c|c|c|c|}
\hline 10.01 & - $\quad 10.0 \mathrm{~J}$ & $m$ & 6.00 & 4.00 & 4.00 & NA & NA \\
\hline$M$ & $m$ & $m$ & 5.00 & 5.04 & 5.04 & NA & NA \\
\hline$M$ & ma & $m$ & 6.0 & 4.0u & 4.00 & HA & NA \\
\hline$M$ & $M$ & $M$ & 4.00 & 4.0u & 4.04 & HA & NA \\
\hline $10 . \alpha$ & - $\quad 10.0$ & $10 . \alpha$ & 4. 01 & 4.00 & 4.04 & 5.00 & 10.00 \\
\hline 10.00 & 10.00 & 10.00 & 4.00 & 4.00 & 4.00 & $10.0 \mathrm{U}$ & 10.04 \\
\hline 5.00 & 5.00 & 5.00 & 15.00 & 15.00 & 15.00 & 5.00 & 5.00 \\
\hline$M$ & $M$ & $M$ & 4.00 & 4.00 & 4.00 & NA & NA \\
\hline MA & NA & HA & 4.00 & 4.00 & 4.00 & NA & NA \\
\hline MA & MA & MA & 16.00 & 14.00 & 14.00 & NA & WA \\
\hline MA & MA & WA & 4.00 & 4.00 & 4.00 & NA & NA \\
\hline MA & MA & KA & 4.00 & 4.00 & 6.00 & MA & NA \\
\hline MA & MA & NA & $4 . \infty$ & 4.04 & 4.00 & NA & HA \\
\hline $\mathrm{MA}$ & HA & KA & 4.00 & 4.00 & 6.00 & NA & NA \\
\hline 5.0 & 5.00 & 5.00 & 4.00 & 4.00 & 4.00 & 5.04 & 5.00 \\
\hline$m a$ & $M$ & MA & 4.0 & 6.00 & 4.00 & NA & NA \\
\hline 5.00 & 5.00 & $5 . \omega$ & 4.00 & 4.00 & 4.00 & 9.0 & 5.04 \\
\hline 5.00 & 5.00 & 0.6 & 6.00 & 4.00 & 4.00 & 5.00 & 5.00 \\
\hline MA & NA & MA & MA & NA & NA & HA. & NA \\
\hline MA & MA & MA & MA & HA & NA & NA & $A$ \\
\hline MA & HA & MA & NA & NA & NA & NA & NA \\
\hline MA & MA & MA & 4.00 & 4.00 & 4.00 & NA & NA \\
\hline 5.00 & 5.00 & 5.00 & HA & MA & NA & 5.00 & 5.00 \\
\hline 40.0 & 35.0 & 36.0 & 33.0 & 22.0 & 31.0 & 470.0 & 54.0 \\
\hline 10.00 & 10.01 & 90.00 & NA & WA & $\mathrm{NA}$ & NA & NA \\
\hline 5.00 & 5.00 & 5.00 & MA & MA & NA & 15.00 & 5.04 \\
\hline
\end{tabular}


KANSAS CITY : SELECTED ANALYSIS FROM KCE5.033-L

Constituent

Sample Date

ug/L

$04 / 23 / 91 \quad 07 / 26 / 91 \quad 10 / 11 / 99$

$1,1,1,2$ - TETRACKLOROETHANE

$1,9,1$ - TRICHLOROETHANE

$1,1,2,2$ - TETRACHLOROE THANE

$1,1,2$ - TRICHLOROETHANE

$1,1,2$-TRICHLOROTRIFLUOROETH

1,1-DICHLOROE THAME

1,9-DICHLOROETHENE

1.9-DICHLOROPROPENE

$1,2,3$-TRICHLOROBERIZENE

$1,2,3$ - TRICHLOROPROPAKE

$1,2,3$-TRIMETHYLEEHZENE

$1,2,4$-TRICHLOROEEN'ZENE

1,2-DIBROMO-3-CHLOROPROPANE

1,2-01 BROMOE THANE

1,2-DICKLOROEEKZEAE

1,2-DICHLOROETHLNE

1,2-DICHLOROETHENE (TOTA1)

1,2-OICHLOROPROPANE

1,3,5-TRIMETHYLBENZENE

1,3-OICHLOROBENZENE

1,3-DICHLOROPROPANE

1,6-DICHLOROBENIZENE

2,2-0ICHLOROPROPANE

2- BUTAHONE

2- EHLOROETHYLVIWYL ETHER

2-CHLOROTOLLENE

2- HEXKNONE

4-CHLOROTOLUENE

4-METHYL-2-PENTANONE

ACETONE

ACROLEIN

ACRYLONITRILE

BENZENE

GROMOBENZENE

BROMOCHLOROMETHKKE

BROMOFORM

CARBOW DISULFIDE

CAREON TETRACHLORIDE

CHLOROBENZENE

CHLORDO I BROMOME THANE

CHLOROETHAKE

CHLOROETHENE

CHLOROFORM

CIS-1,2-DICHLOROETHENE

CI5-1,3-DICKLOROPROPENE

DIEROMOMETHAKE

DICHL OROBROM OME THRME

DICHLORDOIFLUOROMET THANE

ETHYLEEHZENE

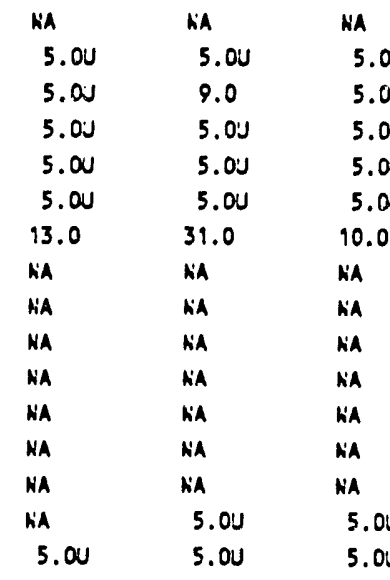

$3100.0 \quad 3300.0 \quad 2500.0$

$\begin{array}{lll}5.00 & 5.00 \quad 5.00\end{array}$

NA NA NA

$\mathrm{NA} \quad 5.00 \quad 5.00$

MA NA NA

HA $\quad 5.00 \quad 5.00$

WA HA HA

$5.00 \quad 5.00 \quad 5.00$

NA NA NA

HA NA NA NA

5.00500500

NA NA NA

5.005005 .005

$10.0 \mathrm{~S} \quad 10.0 \mathrm{~J} \quad 10.0 \mathrm{U}$

NA NA HA

HA HA HA

$5.005 \quad 5.00 \quad 5.00$

MA NA NA NA

NA NA HA

5.0 $5.00 \quad 5.00$

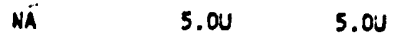

$\begin{array}{lll}5.0 \mathrm{~J} & 5.00 & 5.00\end{array}$

$\begin{array}{lll}5.00 & 5.00 & 5.00\end{array}$

$5.00 \quad 5.00 \quad 5.00$

$10.0 \mathrm{~J} \quad 10.0 \mathrm{~J} \quad 10.0 \mathrm{~J}$

$76.0 \quad 99.0 \quad 290.0$

$\begin{array}{lll}5.0 \mathrm{~J} & 5.0 \mathrm{~J} \quad 5.0 \mathrm{~J}\end{array}$

NA NA NA

$5.0 \mathrm{~S} \quad 5.0 \mathrm{~J} \quad 5.0 \mathrm{~J}$

NA NA NA RA

$5.05 \quad 5.0 \mathrm{~J} \quad 5.00$

NA HA $\mathrm{KA}$

$5.0 \mathrm{~J} \quad 5.0 \mathrm{~J} \quad 5.0 \mathrm{~J}$ 
KANSAS GITY : SELECTED ANALYSIS FROM KC85.033-L

Constituent

us/l

$04 / 23 / 91 \quad 07 / 26 / 91 \quad 10 / 11 / 91$

FLUOROTRICHLORONET MUNE

MEXACKL OROQUTHOIEME

ISOPROPYLBENZEME

M-XYLENE

METHYL EROMIDE

METHYL CNLORIDE

METHYLEME CHLORIDE

M- DUTYLBENZEHE

M-PROPYLEEMZEUE

MAPHTKALEME

O.XYLEME

P-ISOPROPYLTQLUENE

P-XYLENE

SEC-RUTYLBENZENE

STYREXE

TERT-QUTYLLENZENE

TETRACHLOROETHENE

TOLUEME

TPM

TPKH

TPKL

TRUNS-1,2-OICHLOROETHEME

TRANS-1,3-0IGHLOROPROPENE

TRICHLOROETHEME

VIMYL ACETATE

XYLENES (total)

\begin{tabular}{|c|c|c|}
\hline MA & 10.01 & $10 . \alpha$ \\
\hline$M$ & ma & ma \\
\hline$m$ & $m$ & $m$ \\
\hline Ma & $m$ & MA \\
\hline 10.0 & $10 . \omega$ & 10.00 \\
\hline 10.00 & 10.00 & 10.00 \\
\hline s.w & 5.00 & 5.00 \\
\hline$\mu$ & $M$ & $m$ \\
\hline$M A$ & ma & MA \\
\hline MA & NA & MA \\
\hline NA & NA & WA \\
\hline MA & MA & MA \\
\hline MA & HA & HA \\
\hline MA & $M$ & MA \\
\hline s.as & 5.00 & 5.00 \\
\hline MA & NA & MA \\
\hline 110.0 & 260.0 & 220.0 \\
\hline 5.0 & s.as & 5.01 \\
\hline MA & KA & $M A$ \\
\hline $\mathrm{MA}$ & WA & $\mathrm{ma}$ \\
\hline$x_{A}$ & $m$ & MA \\
\hline KA & MA & $m$ \\
\hline $5 . \alpha$ & 5.00 & 5.00 \\
\hline 95.0 & 26000.0 & 6900.0 \\
\hline ma & ma & WA \\
\hline $5.0 \mathrm{~s}$ & $5.0 \mathrm{w}$ & 5.00 \\
\hline
\end{tabular}

Smole Dote 
KANSAS CITY : SELECTED ANALYSIS FROM KCES.033.M

Constituent

us/L

$1,1,1,2$-TETRACKLOROETHANE

$1,1,1$-TRICHLOROE T HAHE

$1,1,2,2$-TETRACKLOROE THANE

$1,1,2$-TRICHLOROET HANE

$1,1,2$ - TRICHLOROTR IFLUOROETH

$1,1-01$ ChLLROE THAHE

9,9 -DICHLOROETKENE

1, 1-DICHLOROPROPENE

1,2,3-TRICHLOROBEKIENE

$1,2,3$-TRICHLOROPROPAHE

$1,2,3$-TRIMETHYLEENZENE

1,2,4-TRICHLOROSERZENE

1,2-DIBROMO-3-CHLOROPROPAHE

1,2-DIBROMOETHAKE

1,2-DICHLOROSEK'ZENE

1,2 -OICKLOROE THANE

1,2-DICMLOROETHENE (total)

1,2-DICHLOROPROPANE

$1,3,5$ - TRIMETHYLBENZENE

1,3-DICHLOROSENZENE

1,3-DICHLOROPROPANE

1,4-DICHLOROSELIENE

$\therefore 2$-DICHLOROPROPANE

2-BUTANONE

2-CHLOROETHYLVIHYL ETHER

2-CHLOROTOLUENE

2- KeXANONE

4- CHLOROTOLUENE

4-METHYL-2-PENTANONE

ACETONE

ACROLEIN

ACRYLONITRILE

BENZENE

BROMOBENZENE

BROMOCHLOROMETHANE

SROMOFORM

CARBON DISULFIOE

CARBON TETRACHLORIDE

CHLOROBENZENE

CHLORDO I BROMOMETHANE

CHLOROETKAKE

CHLOROETHEA'E

CHLOROFORM

CIS-1,2-OICHLOROETHEKE

CIS-1,3-DICHLOROPROPENE

DI BROMOME TKANE

DICHLOROSROMJMETHAKE

OICHLORCOIFLUDROME TKAKE

ETKYLBENZENE

$07 / 28 / 85$

$10 / 27 / 85$

$01 / 31 / 85$

$05 / 07 / 86$

$08 / 04 / 86$

$10 / 27 / 86$

$[2 / 04 / 87$

U

MA

U

NA

U

u

NA

NA

8.2

7.6

NA

NA

hA

WA

WA

NA

NA

NA

11.3

11.3

NA

HA

NA

NA

NA

NA

NA

NA

KA

NA

HA

U

U

HA

NA

HA

in

U

10.00

10.00

HA

NA

NA

U

$14.5 \quad 4.7 \mathrm{~J}$

19.1

NA

NA

HA

NA

HA

NA

NA

HA

22.8

$$
\begin{array}{r}
22.8 \\
\mathrm{NA}
\end{array}
$$

NA

NA

NA

NA

NA

NA

NA

HA

NA

NA

NA

u

U

NA

HA

NA

$$
5.0
$$

NA

WA

NA

iNA

NA

NA

NA

NA

11.

19.9

NA

NA

NA

NA

NA

NA

NA
NA
NA
NA

NA

NA

NA

NA

u

u

10.5

NA $U$

HA

NA

kA

u

3.9

NA

NA

NA

HA

NA

NA

NA

13.9

WA
NA
NA
NA
NA

NA

NA

NA

NA

NA

$$
\begin{array}{r}
U \\
U \\
4.4 U
\end{array}
$$

NA

NA

Ka $u$

24.0
$U$

KA

NA $\begin{array}{r}U \\ U \\ \\ \\ \\ \end{array}$
NA

4

U

NA

NA

U

A

NA

$U$

NA

NA

HA

HA

NA

NA

HA

NA

$32.2^{U}$

NA $U$

NA

NA

NA

NA

NA

NA

NA

NA

NA

NA

$U$

u

NA

NA

NA

34.1

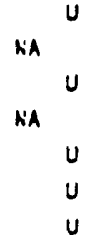

NA

NA

NA

NAA

NA

26.7

KR

NA

NA

NA

NA

NA

NA

NA

NA

$u$

$U$

HA

NA

A

NA

I.A

$\begin{array}{cc}u & u \\ u & u \\ u & u\end{array}$

liA

IA

NA NAA NA

NA NA NA NA

I'A N'A NAA

NA U

WA NA

NA NA

NAA NA

NA NA

NA NA

liA

$\begin{array}{cc}U & U \\ 57.0^{\circ} & 74.0^{\circ}\end{array}$

NA NA

NA NA

NA $\quad K A$

NA

NA

U

NA

NA

iA

NA

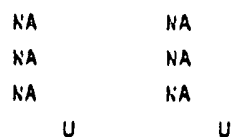

N'A 
KANSAS CITY : SELECTED ANALYSIS FROM KC85.033-M

Constituent

$\omega s / L$

FLLOROTRICHLOROMETHAHE MEXACHLOROQUTNAIENE I SOPRCPYLBENZENE

M-XYLENE

METHYL BROMIOE

METHYL CHLORIDE

METHYLEME CHLORIDE

N-BUTYLBENZENE

W-PROPYLBENZENE

MAPHTHALENE

O-XYLENE

P-I SOPROPYLTOLUENE

P-XYLENE

SEC-BUTYLBENZENE

STYRENE

TERT-BUTYLBENZENE

TETRACHLOROETHENE

TOLUENE

TPH

TPHH

TPHL

TRANS-1,2-01 CHLOROETHENE

TRANS-1,3-DICHLOROPROPENE

TRICHLOROETHENE

VINYL ACETATE

XYLENES (rotal)
07/28/85

$10 / 27 / 85$

Semple Date

$01 / 31 / 88$
$08 / 04 / 86$

$10 / 27 / 86$

$02 / 04 / 37$

\begin{tabular}{|c|c|c|c|c|c|c|c|}
\hline U & u & $u$ & U & $U$ & $u$ & $\mathrm{NA}$ & $\mathrm{NA}$ \\
\hline$M A$ & $M$ & $\mu$ & $M$ & $\mathrm{MA}$ & NA & NA & NA \\
\hline$M$ & $M$ & $M A$ & $M A$ & $\mathrm{MA}$ & $\mathrm{NA}$ & NA & NA \\
\hline$M A$ & $M A$ & $M$ & $M$ & NA & NA & NA & $\mathrm{NA}$ \\
\hline$u$ & $\mathbf{U}$ & $u$ & $u$ & $u$ & $u$ & $u$ & $u$ \\
\hline$U$ & U & $U$ & $U$ & $u$ & U & $u$ & $U$ \\
\hline$u$ & 2.80 & $u$ & 7.9 & 4.8 & $u$ & $u$ & $u$ \\
\hline$x a$ & $M$ & $\mathrm{Mn}$ & $m$ & $\mathrm{NA}$ & in & NA & NA \\
\hline NA & $M$ & MA & $M A$ & MA & NA & $N A$ & NA \\
\hline MA & MA & MA & $\mathrm{KA}$ & NA & NA & NA & NA \\
\hline NA & MA & KA & $\mathrm{HA}$ & NA & NA & NA & NA \\
\hline MA & NA & MA & NA & $\mathrm{NA}$ & NA & $\mathrm{NA}$ & NA \\
\hline$M A$ & NA & $\mathrm{NA}$ & MA & $\mathrm{NA}$ & NA & $N A$ & NA \\
\hline NA & $M A$ & YAA & NA & NA & NA & NA & $\mathrm{HA}$ \\
\hline MA & MA & $m$ & NA & MA & NA & $u$ & $U$ \\
\hline NA & NA & MA & NA & HA & HA & NA & NA \\
\hline$u$ & 4.14 & U & $u$ & $u$ & $u$ & $u$ & u \\
\hline u & $u$ & $u$ & $u$ & $u$ & $u$ & $U$ & $U$ \\
\hline NA & MA & MA & NA & NA & NA & NA & NA \\
\hline MA & $m a$ & $M A$ & MA & MA & NA & NA & H \\
\hline$\mu$ & MA & MA & $M A$ & HA & $\mathrm{NA}$ & NA & $\mathrm{NA}$ \\
\hline MA & MA & MA & MA. & $\mathrm{NA}$ & $\mathrm{NA}$ & NA & $\mathrm{NA}$ \\
\hline U & $u$ & $u$ & $u$ & $u$ & $u$ & $u$ & $u$ \\
\hline 81.5 & 26.4 & 11.7 & 9.6 & 9.2 & 8.6 & $u$ & 37.0 \\
\hline MA & MA & MA & MA & MA & NA & $u$ & $u$ \\
\hline MA & $m a$ & $M A$ & $M A$ & MA & NA & $u$ & $u$ \\
\hline
\end{tabular}


KAK'SAS CITY : SELECTED ANALYSIS FROM KCE5. CI3.M

Const itvent

ug/L
$05 / 01 / 87 \quad 08 / 01 / 87 \quad 10 / 29 / 87$
Sample Date

$01 / 31 / 88 \quad 07 / 26 / 90 \quad 07 / 26 / 91$

\begin{tabular}{|c|c|c|c|c|c|c|}
\hline $1,1,1,2$-TETRACHL OROE THAHE & NA & NA & NA & MA & 4.00 & R'A \\
\hline $1,1,1 \cdot$ TRICHLOROETHANE & $u$ & $1.0 \mathrm{~s}$ & $1.0 \mathrm{~J}$ & 1.00 & 4.00 & 5.00 \\
\hline $1,1,2,2 \cdot$ TETRACKL OROETHANE & $u$ & 1.02 & 1.00 & 1.01 & 4.00 & 5.00 \\
\hline $1,1,2-T R I C H L O R O E T H A N E$ & $u$ & $1.0 j$ & $1.0 \mathrm{~J}$ & $1.0 \mathrm{~J}$ & 4.00 & $5.0 \mathrm{~J}$ \\
\hline 1, 1,2-TRICHLOROTRIFLUOROETH & NA & NA & HA & NA & HA & $5.0 \mathrm{~J}$ \\
\hline 1,1-DICHLOROETHANE & 2.0 & 44.0 & 2.0 & $1.0 \mathrm{~J}$ & 4.00 & 5.00 \\
\hline 1,1-DICHLOROETHEHE & $u$ & 2.0 & $1.0 \mathrm{~J}$ & 6.0 & $7.0 \mathrm{~s}$ & $5.0 \mathrm{U}$ \\
\hline 1,1-DICHLOROPROPENE & NA & NA & NA & NA & 4.00 & KA \\
\hline 1,2,3-TRICHLOROEENZENE & NA & NA & KA & NA & $5.0 \mathrm{~J}$ & NA \\
\hline 1,2,3-TR:CHLOROPROPAKE & NA & NA & NA & HA & $4.0 \mathrm{~J}$ & K'A \\
\hline 1,2,3-TRIMETKYLEENZENE & NA & NA & hiA & h'A & $5.0 \mathrm{~J}$ & K'A \\
\hline $1,2,4$-TRICHLOROBEKIENE & HA & NA & in & HA & 5.00 & NAA \\
\hline 1,2-DIBROMO-3-CKLOROPROPANE & hA & NA & NA & hA & $4.0 \mathrm{~s}$ & H'A \\
\hline 1,2-DIBROMOE THANE & HA & NA & NA & HA & 4.00 & N'A \\
\hline 1,2-DICHLOROBENZENE & HA & HA & NA & NA & $4.0 \mathrm{~J}$ & 5.00 \\
\hline 1,2-DICHLOROETHANE & U & 1.00 & 1.00 & $1.0 \mathrm{~s}$ & $4.0 \mathrm{~V}$ & $5.0 \mathrm{~J}$ \\
\hline 1,2-DICHLOROETHENE (total) & 50.0 & 1.00 & 35.0 & 28.0 & NA & 370.0 \\
\hline 1,2-DICHLOROPROPANE & $\mathbf{U}$ & 1.00 & 1.04 & $1.0 \mathrm{~J}$ & 4.00 & 5.00 \\
\hline 1,3,5-TRIMETHYLBENZENE & HA & NA & NA & NA & $5.0 U$ & NA \\
\hline 1,3-DICHLOROBEKZENE & NA & NA & NA & MA & $4.0 \mathrm{U}$ & 5.00 \\
\hline 1,3-DICHLOROPROPANE & NA & NA & NA & NA & $4.0 \mathrm{~J}$ & NA \\
\hline 1,4-DICHLOROBENZENE & NA & NA & NA & NA & $4.0 \mathrm{U}$ & 5.00 \\
\hline 2,2-DICHLOROPROPANE & NA & NA & NA & NA & 4.00 & NA \\
\hline 2-BUTAKONE & $u$ & $1.0 \mathrm{U}$ & 1.00 & 1.00 & NA & $5.0 \mathrm{U}$ \\
\hline 2-CHLOROETHYLVINYL ETHER & $U$ & 1.00 & 1.00 & $1.0 \mathrm{~s}$ & NA & NA \\
\hline 2-CKLOROTOLUENE & KA & NA & NA & NA & 4.00 & NA \\
\hline 2-HEXANONE & $\mathbf{U}$ & $1.0 \mathrm{~s}$ & $1.0 \mathrm{U}$ & 1.00 & NA & $5.0 \mathrm{U}$ \\
\hline 4-CHLOROTOLUENE & HA & NA & NA & NA & 4.00 & iA \\
\hline 4-METHYL-2-PENTANONE & $\mathbf{U}$ & $1.0 \mathrm{~s}$ & $1.0 \mathrm{~J}$ & 1.00 & NA & 5.00 \\
\hline ACETOWE & NA & MA & NA & NA & NA & $10.0 \mathrm{~J}$ \\
\hline ACROLEIN & NA & NA & NA & NA & NA & N'A \\
\hline ACRYLONITRILE & NA & NA & HA & HA & NA & NA \\
\hline BENZENE & 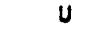 & 1.00 & 1.00 & $1.0 \mathrm{U}$ & 4.00 & 5.00 \\
\hline GROMOSENZENE & NA & MA & NA & NA & 4.00 & NA \\
\hline BROMOCHLOROMETHANE & HA & NA & NA & NA & 4.00 & hA \\
\hline BROMOFORM & $u$ & 1.00 & 1.00 & $1.0 \mathrm{~J}$ & $4.0 \mathrm{U}$ & 5.00 \\
\hline CARBON DISULFIDE & $u$ & $1.0 \mathrm{~J}$ & 1.00 & 1.00 & NA & $5.0 \mathrm{U}$ \\
\hline CARBON TETRACHLORIDE & $\mathbf{U}$ & $1.0 \mathrm{~J}$ & 1.00 & $1.0 \mathrm{~s}$ & 4.00 & $5.0 \mathrm{~J}$ \\
\hline CKLORDSENZENE & $\mathbf{U}$ & 1.00 & 1.00 & 1.00 & 4.00 & $5.0 \mathrm{~J}$ \\
\hline CHLORDO IBROMOME THAHE & $\mathbf{U}$ & 1.00 & 1.00 & 1.00 & 4.00 & 5.00 \\
\hline CHLOROE THANE & $u$ & 1.00 & 1.00 & 1.00 & 4.00 & 10.00 \\
\hline CHLOROETKENE & 18.0 & 18.0 & 9.0 & 15.0 & 11.0 & $i 0.0$ \\
\hline CHLOROFORM & $u$ & $1.0 \mathrm{~J}$ & 1.00 & 1.00 & 4.00 & $5.0 \mathrm{~J}$ \\
\hline CIS-1,2-DICHLOROETHENE & ha & NA & NA & NA & 30.0 & NA \\
\hline CIS-1,3-DICHLOROPROPENE & $\mathbf{U}$ & 1.00 & $1.0 \mathrm{~s}$ & 9.00 & NA & $5.0 \mathrm{~J}$ \\
\hline DIBROMOMETHANE & NA & NA & hiA & NA & 4.00 & HA \\
\hline DICHLOROBROMOME THLNE & $\mathbf{U}$ & 1.00 & $1.0 \mathrm{~s}$ & 1.00 & 4.00 & $5.0 \mathrm{~J}$ \\
\hline DICHLOROOIFLUOROME TKANE & NA & HA & HA & NA & 8.00 & NA \\
\hline ETHYLEENZENE & $U$ & $1.0 \mathrm{~J}$ & $1.0 \mathrm{y}$ & 1.00 & 4.00 & $5.0 \mathrm{~J}$ \\
\hline
\end{tabular}


RANSAS GITY : SELECTED ANALYSIS FROM KC85.033-M

Constituent

ug/L

FLLOROTRICKL OROMETHANE MEXUCKLOROBUTADIENE ISOPROPYLBENZENE

M-XYLEME

METHYL BROWIDE

METHYL CHLORIDE METHYLENE CHLORIDE

N- EUTYLBEXZENE

N-PROPYLDEMZENE

MAPHTHALENE

O-XYLENE

P.1 1 SOPROPYLTOLUENE

P-XYLEKE

SEC-BUTYLBENZENE

STYRENE

TERT-EUTYLBENZENE

TETRACMLOROT THENE

TOLUEME

TPH

TPMH

TPHL

TRUNS-1,2-DICHLOROETHEME

TRAUS-1,3-DICKLOROPROPENE

TRICHLOROETHENE

VIUYL ACETATE

XYLENES CrOta: ;
Semple Date

$\begin{array}{llllll}05 / 01 / 87 & 08 / 01 / 87 & 10 / 29 / 87 & 01 / 31 / 88 & 07 / 26 / 90 & 07 / 26 / 91\end{array}$

\begin{tabular}{|c|c|c|c|c|c|}
\hline$M A$ & $m$ & $\mu$ & $\mu$ & 4.01 & 10.00 \\
\hline$M$ & $M$ & $M$ & $m$ & $5 . \infty$ & MA \\
\hline$M$ & $m$ & $\mu$ & $m$ & 6.01 & NA \\
\hline NA & $m$ & $m$ & $\boldsymbol{M}$ & 4.00 & $\mathrm{NA}$ \\
\hline U & 1.00 & 1.0 & $1 . \alpha$ & 4.00 & 10.00 \\
\hline$u$ & 1.00 & 1.0 & 1.0 & 4.W & 10.04 \\
\hline$u$ & $1 . \infty$ & 3.0 & 1.00 & 15.00 & 5.00 \\
\hline MA & MA & $m$ & M & 6.00 & NA \\
\hline$M$ & MA & $\mathrm{MA}$ & $M$ & 4.00 & NA \\
\hline HA & MA & NA & MA & 14.00 & NA \\
\hline MA & MA & MA & MA & 4.00 & NA \\
\hline MA & MA & HA & NA & 4.00 & NA \\
\hline KA & MA & $M A$ & MA & 4.00 & NA \\
\hline MA & NA & $\mathrm{HA}$ & MA & 4.0u & NA \\
\hline$u$ & 1.00 & $1.0 \mathrm{~s}$ & $1 . \alpha$ & 6.00 & 5.00 \\
\hline KA & NA & MA & ka & 4.04 & MA \\
\hline$u$ & 1.00 & $1 . \alpha$ & 1.00 & 4.00 & 5.00 \\
\hline$u$ & $1 . \omega$ & $1.0 \mathrm{~s}$ & 1.00 & 4.00 & $5.0 \mathrm{U}$ \\
\hline MA & MA & WA & HA & HA & NA \\
\hline MA & NA & $\mathrm{MA}$ & MA & MA & NA \\
\hline MA & MA & KA & MA & NA & NA \\
\hline MA & MA & KA & KA & 4.00 & HA \\
\hline u & $1 . \alpha$ & 1.00 & $1 . \boldsymbol{w}$ & NA & 5.00 \\
\hline U & 1.00 & 1.0 & 2.0 & 4.00 & 870.0 \\
\hline u & 1.00 & 1.00 & 1.00 & NA & NA \\
\hline u & 1.0 & 1.0 & 1.0 & NA & 5.00 \\
\hline
\end{tabular}


KANSAS CITY : SELECTED AKALYSIS IROM KCE5.033.U

Constituent

$\mathrm{ug} / \mathrm{L}$

$n 6 / 13 / \varepsilon 5$

$07 / 28 / 85$

$10 / 27 / 85$

Somple Date

$01 / 31 / 86 \quad 05 / 07 / 86 \quad 08 / 04 / 86 \quad 10 / 27 / 86 \quad 02 / 44 / 87$

\begin{tabular}{|c|c|c|c|c|c|c|c|c|}
\hline 1,1,1,2-TETRACHLOFOE THANE & NA & in & HA & NA & $\mathrm{Na}$ & NA & NA & NA \\
\hline $1,1,1$-TRICHLOROE THANE & $u$ & $u$ & $u$ & $u$ & $u$ & $u$ & $u$ & $u$ \\
\hline 1,1,2,2-TETRACHLOROETHAME & $u$ & $u$ & $u$ & $u$ & $u$ & $u$ & $u$ & $u$ \\
\hline $1,1,2 \cdot T R I C K L O R O E$ THAHE & $u$ & $u$ & $u$ & $u$ & $U$ & $u$ & $u$ & $u$ \\
\hline 1,1,2-TRICHLOROTRIFLUOROETH & in & Na & NA & NA & HA & NA & is & NA \\
\hline 1,1-DICHLOROETKANE & 5.5 & 4.70 & $u$ & $u$ & $u$ & $u$ & $u$ & u \\
\hline 1,1-DICHLOROE THENE & 4.0 & $2.8 \mathrm{U}$ & $u$ & $u$ & $u$ & $u$ & $u$ & $u$ \\
\hline 1,1.O1CHLOROPROPENE & NA & hiA & MA & NA & NA & NA & $\mathrm{NA}$ & iA \\
\hline 1,2,3-TRICHLOROSEKIENE & MA & in & MA & MA & NiA & kin & NA & NA \\
\hline 1,2,3-TRICHLOROPROPAHE & kA & kA & MA & NA & NA & MA & NA & NA \\
\hline 1,2,3-TRIMETHYLLELIZENE & MA & NA & NA & in & NA & K'A & KA & liA \\
\hline 1,2,4-TRICHLOROBENZENE & in & hiA & na & NA & iA & HA & NA & NA \\
\hline 1,2-DIBROMO-3-CHLOROPROPANE & kA & NA & NA & NA & NA & in & i:A & liA \\
\hline 1,2-DIBROMOE THANE & MA & NA & Na & NA & RA & NA & NA & NA \\
\hline 1,2-DICHLOROBENZENE & MA & kA & MA & NA & NA & $\mathrm{NA}$ & NA & iA \\
\hline 1,2-DICHLOROETHANE & $u$ & $u$ & u & u & $u$ & U & $u$ & u \\
\hline 1,2-DICHLOROETHENE (total) & 8.2 & 13.8 & 8.7 & $u$ & $u$ & 5.0 & 18.0 & 16.0 \\
\hline 1,2-DICHLOROPROPAHE & u & u & $u$ & $u$ & $u$ & U & $u$ & u \\
\hline 1,3,5-TR JME THYLLBE KZENE & NA & NA & NA & nA & NA & NA & NA & NA \\
\hline 1,3-DICHLOROBENZENE & NA & NA & ka & NA & NA & MA & iA & NA \\
\hline 1,3-DICHLOROPROPANE & NA & NA & kA & NA & NA & MA & NA & NA \\
\hline 1,4-DICHLOROBENZELE & NA & HA & NA & MA & NA & MA & NA & NA \\
\hline 2,2-DICHLOROPROPANE & MA & NA & in & NA & MA & MA & NA & NA \\
\hline 2-BUTANOWE & WA & MA & MA & NA & KA & MA & $u$ & $u$ \\
\hline 2-CHLOROETHYLVIUYL ETHER & $u$ & $u$ & u & $u$ & $u$ & $u$ & $u$ & $u$ \\
\hline 2-CHLOROTOLUENE & in & kA & KA & HA & NA & in & NA & NA \\
\hline 2-HEXANOWE & MA & NiA & NA & NA & in & in & U & $u$ \\
\hline 4-CHLOROTOLUENE & NA & MA & Na & MA & NA & NA & NA & NA \\
\hline 4-METHYL-2-PENTANOWE & MA & iA & MA & NA & HA & NA & $u$ & $u$ \\
\hline ACETONE & MA & NA & NA & KA & NA & NA & NA & SA \\
\hline ACROLEIN & $u$ & $u$ & $u$ & $U$ & $u$ & $u$ & NA & NA \\
\hline ACRYLONITRILE & $u$ & $u$ & $u$ & $u$ & $u$ & $u$ & NA & NA \\
\hline BENZENE & $u$ & $u$ & u & $4.4 U$ & $u$ & $u$ & $u$ & $u$ \\
\hline BROMOBENZENE & $\mathrm{ka}$ & NA & NA & NA & NA & NA & BA & NA \\
\hline BROMOCHLOROMETHANE & NA & in & NA & NA & HA & NA & $\mathrm{NA}$ & $\mathrm{NA}$ \\
\hline BROMOFORM & $u$ & $u$ & $u$ & U & $u$ & $u$ & $u$ & $u$ \\
\hline CARBON DISULFIDE & kA & MA & NA & WA & NA & MA & $u$ & $u$ \\
\hline CARBON TETRACHLORIDE & $u$ & $u$ & $U$ & $u$ & $u$ & $u$ & $u$ & $u$ \\
\hline CHLOROBEKZENE & $u$ & $u$ & $u$ & $u$ & $u$ & $u$ & $u$ & $u$ \\
\hline CHLORDO I GROMOMET THAKE & $u$ & $u$ & $u$ & u & $u$ & $u$ & $u$ & $u$ \\
\hline CHLOROETHANE & $u$ & $u$ & $u$ & $u$ & u & $v$ & $u$ & u \\
\hline CHLOROE THENE & $10.0 \mathrm{~J}$ & $u$ & u & u & $10.0 \mathrm{~J}$ & U & $u$ & $u$ \\
\hline CHLOROFORM & u & 2.4 & 2.3 & 4.7 & 5.4 & 5.8 & 4.0 & 2.0 \\
\hline CIS-1,2-OICHLOROETHEHE & NA & in & HA & kiA & in & kA & NA & NA \\
\hline CIS-1,3-DICHLOROPROPEKE & $u$ & $u$ & $U$ & $u$ & $u$ & $u$ & u & $u$ \\
\hline DIBROMOMETHRNE & NA & MA & HA & NA & NA & KA & NA & NA \\
\hline DICHL OROBROMDME THANE & $u$ & $u$ & $U$ & u & $u$ & $u$ & U & $u$ \\
\hline DICHLORCO I FLUSROME T HAHE & $u$ & u & $u$ & u & $u$ & $u$ & liA & NA \\
\hline EIHYLEENZENE & u & $u$ & $u$ & $u$ & $u$ & u & u & u \\
\hline
\end{tabular}




$$
C-11
$$

raNSAS CIIY : SELECTED ANALYSIS FROM KCES.033.U

Conet ltuent

uosth

FlUDRotRICKL CRONE THAME MEXACHL CROOUTADIEME ISOPROPYLEERENE

M.XYLENE

METHYL BRONIOE

METKYL CHLORIOE

METHYLEAE CHLORIOE

M-BUTYLSENZENE

n-Proprlaenzene

NAPHTHALENE

O-XYLEKE

P-I SOPROPYLIOLUENE

P-XYLEGE

SEC-BUTYLBENZENE

STYRENE

TERT-QUTYLBENZENE

TETRACHLOROETHENE

TOLUENE

TPH

TPКн

TPHL

TRAMS-1,2-DICHLOROETHENE TRUAS-1,3-DICKLOROPROPENE IRICHLOROETHENE VINTL ACETATE

XYLEMES (rotol)

\section{$06 / 13 / 85$}

$07 / 28 / 85$

$10 / 27 / 85$

Sumple Dote

$01 / 31 / 86$. $05 / 07 / 86 \quad 08 / 04 / 86 \quad 10 / 27 / 86 \quad 02 / 06 / 27$

\begin{tabular}{|c|c|c|c|c|c|c|c|}
\hline$U$ & $\mathbf{u}$ & $U$ & $u$ & $\mathbf{U}$ & $U$ & NA & KA \\
\hline 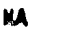 & MA & MA & $M$ & $M A$ & MA & HA & HA \\
\hline$m$ & $\mu$ & $\mu$ & $m$ & MA & KA & kA & HA \\
\hline$m$ & $m$ & $m$ & $\omega$ & MA & HA & NA & HA \\
\hline$U$ & $U$ & $u$ & $u$ & $u$ & $u$ & $u$ & u \\
\hline$u$ & $u$ & $\mathbf{u}$ & $U$ & $u$ & $u$ & u & $u$ \\
\hline$u$ & 5.1 & 13.6 & 8.0 & $u$ & $u$ & $u$ & $u$ \\
\hline MA & $m$ & MA & $M$ & WA & MA & NA & NA \\
\hline$M A$ & $M$ & MA & $M A$ & HA & NA & HA & NA \\
\hline$\mu$ & MA & NA & NA & MA & NA & HA & NA \\
\hline KA & MA & MA & WA & NA & MA & NA & NA \\
\hline MA & MA & MA & NA & NA & NA & NA & KA \\
\hline$* A$ & KA & ka & WA & MA & NA & NA & NA \\
\hline MA & $M A$ & $M A$ & MA & WA & MA & NA & NA \\
\hline NA & MA & NA & NA & KA & HA & $u$ & U \\
\hline MA & MA & MA & MA & MA & HA & NA & NA \\
\hline $\mathbf{u}$ & 5.1 & 4.10 & 4.10 & $u$ & $u$ & $U$ & $u$ \\
\hline$U$ & $u$ & $u$ & $\mathbf{U}$ & $\mathbf{u}$ & $U$ & $U$ & $u$ \\
\hline$M$ & MA & NA & KA & $N A$ & MA & NA & NA \\
\hline MA & MA & MA & MA & NA & $M A$ & NA & $\wedge$ \\
\hline MA & NA & KA & NA & WA & KA & NA & $k A$ \\
\hline MA & WA & MA & MA & MA & NA & KA & $A A$ \\
\hline$u$ & $u$ & $u$ & $U$ & $u$ & $U$ & $U$ & U \\
\hline 63.7 & 160.0 & 86.6 & 52.7 & 70.7 & 35.0 & 38.0 & 37.0 \\
\hline$m$ & $m$ & $m$ & MA & Ma & NA & $u$ & $u$ \\
\hline MA & $m$ & WA & MA & MA & MA & $u$ & $u$ \\
\hline
\end{tabular}


KANSAS CITY : SELECIED AKALLYIS FROM KCE5.033.U

Constituent

Ua/L
Semple Date

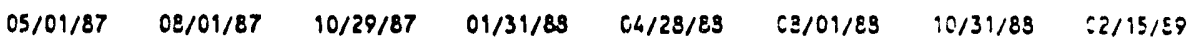

\begin{tabular}{|c|c|c|c|c|c|c|c|c|}
\hline 1,1,1,2-TETRACHLOROE TKANE & NA & $\mathrm{NA}$ & NA & MA & NA & HA & iA & SA \\
\hline 1,1,1-TRICHLOROETHANE & $u$ & 1.00 & 1.00 & $1 . \infty$ & $1.0 \mathrm{U}$ & $1.0 \mathrm{~s}$ & 1.00 & 5. C.J \\
\hline 1,1,2,2-TETRACHL OROETHANE & $u$ & 1.00 & 1.00 & 1.00 & $1.0 \mathrm{~J}$ & $1.0 \mathrm{U}$ & $1.0 \mathrm{~J}$ & $5.0 \mathrm{~J}$ \\
\hline 1,1,2-TRICHLOROE THAHE & $u$ & $1.0 \mathrm{~s}$ & 1.00 & $1.0 \mathrm{~J}$ & $1.0 \mathrm{~J}$ & $9.0 \mathrm{~J}$ & 1.00 & 5.010 \\
\hline 1,1,2-TRICHLOROTRIFLUOROETH & in & NA & NA & NA & NA & NA & KA & NA \\
\hline 1.9-DICHLOROETKANE & 1.0 & 1.00 & 2.0 & 12.0 & $1.0 \mathrm{~J}$ & $1.0 \mathrm{~s}$ & 9.00 & 5.00 \\
\hline 1,1-DICHLOROE THENE & u & $1.0 \mathrm{~J}$ & 1.00 & 1.00 & 1.00 & $1.0 \mathrm{~J}$ & 1.04 & $5.0 \mathrm{cs}$ \\
\hline 1,1-DICHLOROPROPENE & HA & NiA & MA & hiA & Wh & KA & NA & iA \\
\hline $1,2,3$-1RICHLOROBENZENE & in & NA & MA & NA & HA & NiA & SA & lia \\
\hline $1,2,3$-TRICHLOROPROPANE & NA & NA & wa & nA & NA & in & NA & NA \\
\hline 1,2,3-TRJMETHYLLENZENE & MA & MA & his & NA & NA & NA & NA & NA \\
\hline $1,2,4-T R I C H L O R O B E N 2$ ENE & MA & NA & hiA & NA & NA & in & NA & hA \\
\hline 1,2-DIBROMO-3-CHLOROPROPALE & HA & HA & his & NA & NA & HA & NA & NA \\
\hline 1.2-DIBROMOE TKANE & in & NA & MA & MA & NA & NA & NA & N'A \\
\hline 1.2-DICHLOROBEHZENE & kA & NA & NA & NA & NA & KA & N'A & NA \\
\hline 1,2-DICKLOROETHANE & u & 1.00 & 1.00 & 1.00 & $1.0 \mathrm{~J}$ & 1.00 & $1.0 \mathrm{~s}$ & $5.0 \mathrm{~J}$ \\
\hline 1,2-DICHLOROETKENE (tOTAl) & 14.0 & 16.0 & 26.0 & 49.0 & 19.0 & 7.0 & 15.0 & 8.0 \\
\hline 1,2-DICHLOROPROPAHE & u & 1.00 & 1.00 & 1.00 & 1.00 & 1.00 & $1.0 \mathrm{~J}$ & 5.00 \\
\hline 1,3,5-TRIMETHYLEENZENE & MA & NA & MA & NA & in & NA & NA & NA \\
\hline 9,3-DICHLOROBENZENE & NA & Na & MA & kA & MA & $\mathrm{NA}$ & NA & NA \\
\hline 1,3-DICHLOROPROPANE & NA & NA & NA & kA & NA & kir & NA & NA \\
\hline 1,4-DICHLOROBENZENE & in & nA & MA & MA & NA & NA & NA & NA \\
\hline 2,2-DICHLOROPROPANE & MA & NA & HA & kA & MA & NA & NA & NA \\
\hline 2-BUTANOWE & $u$ & 1.00 & 1.00 & $1 . \infty$ & 1.00 & $1.0 \mathrm{~J}$ & 1.00 & 10.0 .4 \\
\hline 2-CHLOROCTHYLVINYL ETHER & $u$ & 1.00 & 1.04 & $1.0 \mathrm{~J}$ & 1.00 & $1.0 \mathrm{~J}$ & $1.0 \mathrm{U}$ & 10.0 .0 \\
\hline 2-CHLOROTOLUENE & MA & NA & Na & Na & NA & NA & NA & NA \\
\hline 2-HEXAKONE & $u$ & 1.00 & $1 . \alpha$ & 1.00 & 1.00 & 1.00 & 1.00 & $10.0 U$ \\
\hline 4-CMLOROTOLUENE & WA & WA & Na & Ha & HA & HA & NA & NA \\
\hline 4-KETHYL-2-PENTANONE & $u$ & $1 . \infty$ & $1.0 \mathrm{~s}$ & 1.00 & 1.00 & 1.00 & 1.00 & 10.01 \\
\hline ACE TOWE & MA & NA & NA & NA & NA & NA & NA & $10.2 U$ \\
\hline ACROLEIN & MA & MA & MA & kA & MA & NA & in & $100.8 \mathrm{U}$ \\
\hline ACRYLONITRILE & NA & NA & NA & KA & in & NA & NA & $100.8 \mathrm{~J}$ \\
\hline BENZENE & $u$ & 1.00 & $1.0 \mathrm{~s}$ & 1.00 & 1.00 & 1.04 & 1.00 & $5.0 \mathrm{~J}$ \\
\hline BROMOBENZENE & NA & HA & NA & NA & NA & NA & $\mathrm{NA}$ & NA \\
\hline BROMOCHLOROME THAHE & NA & NA & NA & in & NA & NA & K'A & KA \\
\hline BROMOFORK & $u$ & 1.00 & 1.00 & 1.00 & 1.00 & $1.0 \mathrm{~J}$ & 1.04 & 5.00 \\
\hline CARBON DISULFIDE & $u$ & 1.00 & 1.00 & 1.00 & $1.0 \mathrm{~s}$ & $1.0 \mathrm{~s}$ & $1.0 \mathrm{~J}$ & 5.0 .5 \\
\hline CARBOW TETRACHLORIDE & u & 1.00 & 1.00 & 1.00 & 1.04 & $1.0 \mathrm{~s}$ & 1.00 & 5.04 \\
\hline CHLOROBENZENE & u & 1.00 & 1.00 & 1.00 & 1.00 & 1.00 & 1.01 & $5.0 \mathrm{~J}$ \\
\hline CHLORDO I SROMOMET THANE & $u$ & 1.00 & 1.00 & 1.00 & 1.00 & $1.0 \mathrm{~J}$ & 1.00 & 5.04 \\
\hline CHLOROETKANE & u & 1.00 & 1.00 & $1.0 \mathrm{u}$ & $1.0 \mathrm{~J}$ & 1.04 & 1.00 & $10.0^{\prime \prime}$ \\
\hline CHL OROETHENE & 2.0 & $1.0 \mathrm{~s}$ & 18.0 & 3.0 & 2.0 & 3.0 & 7.0 & $10.2 \mathrm{~J}$ \\
\hline CHLOROFORN & 2.0 & 1.0 & 1.00 & 1.0 .1 & $1.0 \mathrm{~s}$ & 1.c'J & 1.JJ & 5.90 \\
\hline CIS-1,2-DICHLOROE THENE & NA & in & Nh & his & NA & NA & N'A & NA \\
\hline CIS-1,3-DICHLOROPROPENE & u & 1.00 & 1.00 & $1.0 \mathrm{~J}$ & $1.0 \mathrm{~J}$ & $1.0 \mathrm{v}$ & 1.00 & $5.0 \mathrm{~J}$ \\
\hline DI GROMJMETKRANE & NA & KA & NA & NA & hA & NA & NA & NA \\
\hline DICHLOROBROMOMET TKANE & $U$ & 1.00 & 1.00 & 1.00 & $1.0 \mathrm{~J}$ & 1.C.J & $1.0 \mathrm{~J}$ & $5.01 \mathrm{~J}$ \\
\hline DICHLORDO I FL LOROME T TLKE & in & NA & NA & NA & NA & IIA & NA & NA \\
\hline ETHYLBENZENE & $u$ & 1.00 & $1.0 \mathrm{~s}$ & 1.00 & 1.00 & 1.00 & $1.0 \mathrm{~s}$ & 5.01 \\
\hline
\end{tabular}




\section{C-16}

RANSAS CITY : SELECTED AMALYSIS FROM KC85.033.U

Constituent

$\omega o / L$

PLLOROTRICHLORONETHANE MEXACHLOROQUTAD IETEE I SOPROPYLIENZENE

M-XYLEKE

METHYL BROMIDE

METHYL CHLORIDE

METHYLENE CHLORIDE

$M$ - UUTYLBENZENE

M-PROPYLBENZEME

MAPHTHALENE

O-XYLENE

P-1 SOPROPYL TOLUENE

P-XYLENE

SEC-RUTYLBENZENE

STYRENE

PERT-BUTYLBENZENE

TETRACHLOROETHEME

TQLUEME

TPY

TPHM

TPHL

TRAMS-1, 2-DICHLOROE THENE TRANS-1,3-DICHLOROPROPENE

TRICHLOROETHENE

VIMYL ACETATE

XYLENES (total)

\begin{tabular}{|c|c|c|}
\hline MA & $M$ & $M$ \\
\hline$M$ & $M$ & $m$ \\
\hline$M A$ & $M$ & $M$ \\
\hline$m$ & $\mathrm{ma}$ & $M$ \\
\hline$u$ & 1.00 & 1.0 \\
\hline$u$ & $1 . \omega$ & 1.0 \\
\hline$u$ & 1.00 & 3.0 \\
\hline$M$ & MA & $M A$ \\
\hline$M A$ & MA & $M$ \\
\hline NA & WA & MA \\
\hline MA & MA & NA \\
\hline$M A$ & NA & MA \\
\hline MA & MA & MA \\
\hline$M A$ & MA & MA \\
\hline u & 1.00 & 1.0 \\
\hline$M$ & MA & $M A$ \\
\hline 1.0 & 1.00 & 1.0 \\
\hline$u$ & 1.00 & 1.00 \\
\hline$\mu_{A}$ & $M A$ & NA \\
\hline MA & $m$ & $M A$ \\
\hline$M A$ & MA & MA \\
\hline MA & $M A$ & MA \\
\hline u & 1.00 & $1 . \infty$ \\
\hline 33.0 & 23.0 & 23.0 \\
\hline U & 1.00 & 1.0 \\
\hline$u$ & $1 . w$ & 1.00 \\
\hline
\end{tabular}

$M A$
$M A$
$M A$
1.00
28.0
$M A$
$M A$
$M A$
$M A$
$M A$
$M A$
1.00
$M A$
1.00
1.00
$M A$
$M A$
$M A$
$1.0 U$
32.0
$1.0 U$
1.00

$08 / 01 / 88$

$10 / 31 / 88$

$02 / 15 / 89$

$05 / 01 / 87$

$08 / 01 / 87$

10/29/87

$01 / 31 / 88 \quad 06 / 28 / 88$

08/01/8s

MA

NA

$\begin{array}{ll}\text { NA } & 10.00 \\ \text { NA } & \text { NA } \\ \text { NA } & \text { NA } \\ \text { NA } & \text { NA } \\ 1.00 & 10.04 \\ 1.00 & 10.00 \\ 1.00 & 5.00\end{array}$

1.00

1.00

1.04

1.00

5.00

in

MA

WA

MA

NA

NA

1.00

NA

1.00

1.00

MA

NA

KA

1.00

12.0

1.00

NA

NA

NA

NA

NA

NA

NA

1.00

MA

1.04

1.00

NA

NA

MA

HA

1.00

4.0

1.04

NA

NA

NA

NA

NA

HA

NA

1.00

NA

1.00

1.00

NA

NA

HA

NA

1.04

11.0

1.00

NA

NA

NA

NA

HA

NA

NA

S.0U

NA

$5.0 \mathrm{~s}$

5.00

$$
\text { NA }
$$

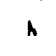

NA

NA

5.00

6.0

1.00

1.00

1.00

10.00 
KANSAS CITY : SELECTED ANALYSIS FROM KCES-033-U

Constifuent

ug/L

05/01/89

Sample Dote

$02 / 06 / 90 \quad 07 / 26 / 90$

\begin{tabular}{|c|c|c|c|c|c|}
\hline 1,1,1,2-TETRACHLOROE THANE & his & $\mathrm{MA}$ & MA & 4.00 & 4.00 \\
\hline 1,1,1-TRICHLOROETKAKE & 5.00 & 5.00 & 5.04 & $4.0 \mathrm{U}$ & 4.0 .5 \\
\hline 1,1,2,2- TETRACHLOROETHANE & 5.04 & 5.00 & 5.00 & $4.0 \mathrm{u}$ & 4.0 .5 \\
\hline 1,1,2-TRICHLOROETKRHE & 5.00 & $5.0 \mathrm{~s}$ & 5.00 & 4.0 & $4.0 \mathrm{~J}$ \\
\hline 1,1,2-TRICKL OROTRIFLUOROETH & MA & NiA & NA & NA & ha \\
\hline 1,1-DICHLOR DETKALEE & $5.0 \mathrm{~J}$ & 5.0 .1 & 7.0 & 18.0 & 21.0 \\
\hline 1,1-DICHLOROETHENE & $5.0 \mathrm{~J}$ & 5.00 & 5.00 & $7.0 \mathrm{~J}$ & $7.0 \mathrm{~J}$ \\
\hline 1,1-DICHLOROPROPENE & in & kA & kA & 4.00 & 4.0.J \\
\hline 1,2,3-TRICHL OROBER'ZENE & wA & KA & kA & $5.0 \mathrm{~J}$ & $5.0 \mathrm{~s}$ \\
\hline $1,2,3 \cdot$ TRICHLOROPROPAHE & in & in & in & $4.0 \mathrm{~J}$ & 4.00 \\
\hline 1,2,3-TRIME THYL BEKZENE & MA & in & HA & 5.00 & $5.0 \mathrm{~J}$ \\
\hline $1,2,4 \cdot T R I C H L O R O B E K Z E N E$ & MA & iA & MA & 5.00 & 5.00 \\
\hline 1.2-01GROMO-3.CHLOROPROPRLE & in & in & M.A & $4.0 \mathrm{~J}$ & $4.0 \mathrm{~J}$ \\
\hline 1.2-D1BROMOE THALE & in & Ha & in & $4.0 \mathrm{~J}$ & 4.0 .5 \\
\hline 1,2-OICHLOROSENLENE & in & NA & in & 4.00 & $6.0 \mathrm{~J}$ \\
\hline 1,2-DICHLOROE THANE & $5.0 \mathrm{~J}$ & 5.00 & 5.00 & 4.04 & 4.00 \\
\hline 1,2-OICHLOROETHENE (IOTOL) & 12.0 & 5.0 & 6.0 & MA & NA \\
\hline 1,2-DICHLOROPROFANE & 5.00 & 5.00 & 5.00 & $4.0 \mathrm{U}$ & 4.00 \\
\hline 1,3,5-TRIME T HYL BEN'ZENE & NA & NA & NA & 5.00 & $5.0 \mathrm{~J}$ \\
\hline 1,3-DICHLOROBEHLENE & HiA & NA & MA & 4.00 & 4.00 \\
\hline 1,3-DICHLOROPROPANE & ka & na & NA & 6.00 & 4.015 \\
\hline 1,4-DICHLOROSENZENE & MA & na & Na & 4.00 & 4.00 \\
\hline 2,2-DICHLOROPROPAHE & iA & Na & NA & 4.00 & $4.0 \mathrm{~J}$ \\
\hline 2-BUTAMONE & 10.0.5 & 10.00 & 10.00 & NA & MA \\
\hline 2-CHLOROETHYLVIHYL ETHER & 10.00 & $10.0 \mathrm{~s}$ & NA & kA & NA \\
\hline 2-CHLOROTOLUERE & Na & NA & HA & $4.0 \mathrm{~J}$ & 4.00 \\
\hline 2- HEXAHOWE & 10.00 & 10.00 & 10.00 & HA & in \\
\hline 4-CHLOROTOLUENE & NA & NA & NA & 4.05 & 4.00 \\
\hline 4-METHYL-2-PENTANONE & 10.00 & 10.00 & 10.00 & na & MA \\
\hline ACETONE & 10.04 & $=\quad 10.00$ & 10.00 & NA & MA \\
\hline ACROLEIH & $100.0 \mathrm{~J}$ & - $\quad 100.00$ & HA & in & in \\
\hline ACRYLONITRILE & 100.00 & 100.00 & NA & AA & kn \\
\hline GENZENE & 5.00 & 5.00 & 5.00 & 4.05 & 4.00 \\
\hline BROMOBENZENE & NA & HiA & MA & 4.00 & 4.00 \\
\hline BROMOCHLOROMETHANE & HA & NA & NA & $4.0 \mathrm{~J}$ & 4.00 \\
\hline BROMOFORM & $5.0 \mathrm{~J}$ & 5.00 & $5.0 \mathrm{~J}$ & 4.00 & $4.0 \mathrm{~J}$ \\
\hline CARBOW DISULFIDE & 5.00 & 5.00 & 1.0 & NA & NA \\
\hline CARBOW TETRACHLORIDE & 5.00 & 5.00 & $5.0 \mathrm{~J}$ & $4.0 \mathrm{~s}$ & 4.011 \\
\hline CHLOROSENZENE & 5.04 & 5.04 & 5.00 & 4.04 & 4.00 \\
\hline CKLORDOI I BROMOMETKANE & 5.015 & 5.00 & 5.00 & 4.00 & 4.00 \\
\hline CMLOROETHANE & $10.0 \mathrm{~J}$ & 10.00 & 90.00 & $4.0 \mathrm{~J}$ & 4.00 \\
\hline CKL OROETHENE & $10.0 \mathrm{~J}$ & $10.0 \mathrm{~J}$ & 10.00 & 4.04 & 4.00 \\
\hline CHLOROFORM & $5.0 \mathrm{~J}$ & 5.00 & 5.00 & 4.00 & 4.00 \\
\hline CIS-9,2-DICHLOROETHENE & NA & NA & WA & 6.0 & 6.0 \\
\hline C15-1,3-DICMLOROPROPENE & $5.0 \mathrm{~J}$ & 5.00 & 5.00 & NA & sa \\
\hline DIBROMOME THANE & NA & NiA & Na & 4.00 & $4.0 \mathrm{~s}$ \\
\hline DICHLOROBROMOME THANE & $5.0 \mathrm{~J}$ & 5.00 & 5.00 & $4.0 \mathrm{~J}$ & $4.0 \mathrm{~s}$ \\
\hline DICHLORDOIFLUOROMETHANE & in & NA & KA & $8.0 \mathrm{~J}$ & 8.0 .5 \\
\hline ETKYLEENZENE & $5.0 \mathrm{~J}$ & 5.00 & 5.00 & $4.0 \mathrm{~s}$ & $4.0 \mathrm{~J}$ \\
\hline
\end{tabular}


KANSAS CITY : SELECTED ANALYSIS FROM KC85.033.U

Constituent

$\omega g / L$

FLUOROTRICHLORONETHANE MEXUCHLOROQUTADIENE ISOPROPYLBENZEME M-XYLENE METHYL BROMIDE METHYL CHLORIDE METHYLENE CHLORIDE N-BUTYLBENZENE M-PROPYLBENZENE MAPHTHALENE

O-YYLENE P-I SOPROPYLTOLUENE P-XYLENE' SEC-BUTYLBERZENE STYRENE TERT-EUTYLBENZENE TETRACHLOROETKEKE YOLUENE

TPH

TPHH

TPHL

TRUAS-1,2-DIEHLOROETHENE TRANS-1,3-0ICHLOROPROPENE TRICHLOROETHENE VIMYL ACETATE XYLENES (total)

\begin{tabular}{|c|c|c|c|c|}
\hline 10.0 & $-\quad 10.00$ & ma & 6.00 & 6.00 \\
\hline M & $M$ & MA & 5.00 & $5.0 \mathrm{~s}$ \\
\hline$M$ & $M$ & $M$ & 4.00 & 4.00 \\
\hline M & $m$ & $M$ & 6.00 & 4.00 \\
\hline 10.00 & 10.00 & 10.00 & h.ov & 4. $\omega$ \\
\hline 10.0 & 10.00 & 10.00 & 6.00 & 6.00 \\
\hline $5 . \omega$ & 5.00 & 3.0 & 15.00 & 15.00 \\
\hline$M$ & $M$ & $M$ & 4. 0 & 4.00 \\
\hline wa & $M$ & MA & 6.00 & 4.00 \\
\hline HA & MA & MA & 16.00 & 14.00 \\
\hline NA & MA & MA & 4.00 & 4.04 \\
\hline NA & NA & NA & 4.00 & 4.00 \\
\hline NA & KA & KA & 4.00 & 4.00 \\
\hline NA & MA & NA & $4.0 \mathrm{~J}$ & 4.00 \\
\hline 5.00 & 5.00 & $5 . \infty$ & 4.00 & 4.00 \\
\hline NA & MA & NA & 4.00 & 4.00 \\
\hline $5.0 \mathrm{~s}$ & 5.00 & 5.00 & 4.00 & 4.00 \\
\hline 5.00 & 5.00 & 5.00 & 4.00 & 4.00 \\
\hline MA & MA & $M A$ & NA & NA \\
\hline MA & MA & MA & NA & MA \\
\hline MA & NA & $\mathbf{M A}$ & MA & NA \\
\hline MA & HA & NA & 4.00 & 4.00 \\
\hline $5 . \omega$ & 5.00 & 5.00 & NA & KA \\
\hline 15.0 & 5.00 & 2.0 & 4.00 & 4.00 \\
\hline $10.0 \mathrm{~J}$ & 10.00 & 10.00 & MA & NA \\
\hline $5 . \omega$ & 5.00 & 5.00 & NA & NA \\
\hline
\end{tabular}


KANSAS CITY : SELECTED AKRLYSIS FROM KCES.044

Constituent

US/L

$07 / 28 / 85$

Sample Date

$08 / 04 / 86 \quad 02 / 04 / 87 \quad 10 / 31 / 88 \quad 02 / 11 / 89 \quad 07 / i 2 / 90$

\begin{tabular}{|c|c|c|c|c|c|c|c|c|}
\hline $1,1,1,2$ - TETKACHLOROETHANE & NA & MA & MA & NA & NA & NA & NA. & 4.0 .1 \\
\hline 1,1,1-TRICHLOROETHRHE & u & $U$ & $u$ & $u$ & $u$ & 1.00 & $5.0 \mathrm{~J}$ & $4.0 \mathrm{~J}$ \\
\hline 1,1,2,2-TETRACHLOROETHANE & $u$ & $u$ & $u$ & $u$ & $u$ & 1.00 & 5.00 & $4.0 \%$ \\
\hline 1,1,2-TR!CHLOROET THANE & $u$ & u & $u$ & $U$ & $u$ & 1.00 & $5.0 \mathrm{~J}$ & 4.00 \\
\hline 1,1,2-TRICHLOROTRIFLWOROETH & in & NA & NA & HA & NA & NA & NA & NA \\
\hline 1,1-DICHLOROETHANE & $u$ & $u$ & $u$ & $u$ & $u$ & 1.00 & 5.00 & $4.0 \mathrm{~J}$ \\
\hline 1,1-DICHLOROETKEKE & $u$ & $u$ & $u$ & $U$ & $u$ & 1.CJ & 5.04 & $7.0 \mathrm{~J}$ \\
\hline 1,1-DICHLOROPROPENE & HA & in & kA & HA & NA & HA & NA & $4.0 \mathrm{v}$ \\
\hline 1,2,3-TRICHLOROBEAZENE & Hil & in & kA & NA & NA & NA & NA & 5.015 \\
\hline $1,2,3$-TRJCHLOROPROPANE & in & MA & NA & HA & NA & NA & NA & 4.05 \\
\hline 1,2,3-TRIMETHYLBEK'ZEKE & NA & ki & NA & in & NA & NA & NA & $5.0 \mathrm{~J}$ \\
\hline 1,2,4-TRICHLOROBEK'ZENE & Ma & NA & MA & nA & NA & NA & NA & 5.00 \\
\hline 9,2-DISROMO-3-CHLOROPROPANE & NA & NA & NA & SiA & NA & N'A & NA & 4.00 \\
\hline 1,2-015ROMOETHANE & His & MA & NA & kA & NA & NA & NA & 4.00 \\
\hline 9,2-DICHLOROEEKZENE & NA & Wh & MA & NA & NA & HA & NA & 4.00 \\
\hline 1,2-DICHLOROETHANE & $u$ & $u$ & $u$ & $u$ & $u$ & 1.00 & 5.00 & 4.00 \\
\hline 1,2-DICHLOROETHENE (total) & $u$ & u & $u$ & $u$ & 1.0 & 1.04 & 5.00 & NA \\
\hline 1,2-DICHLOROPROPANE & u & $u$ & $u$ & $U$ & $u$ & 1.00 & $5.0 \mathrm{~J}$ & 4.00 \\
\hline 1,3,5-TRIMETHYLBENZENE & NA & MA & NA & NA & NA & HA & NA & 5.00 \\
\hline 1,3-DICKLOROBEKZENE & NA & MA & MA & HA & HA & NA & NA & 4.05 \\
\hline 1,3-DICHLOROPROPANE & MA & wa & MA & NA & NA & NA & NA & 4.01 \\
\hline 1,6-DICHLOROBENZENE & NA & NA & NA & nA & NA & MA & NA & 4.00 \\
\hline 2,2-DICHLOROPROPANE & KA & Na & in & Na & NA & NA & NA & 4.0 .5 \\
\hline 2-BUTANONE & nA & MA & MA & Na & U & 9.00 & 10.00 & N'A \\
\hline 2-CHLOROETHYLVINYL ETHER & u & u & $u$ & $u$ & $u$ & 1.00 & 10.00 & kA \\
\hline 2-CHLOROTOLUENE & KA & Ma & NA & nA & MA & NA & NA & 4.04 \\
\hline 2- HEXANONE & kA & NA & NA & MA & $u$ & 9.00 & 10.04 & NA \\
\hline 4-CHLOROTOLUENE & MA & NA & NA & NA & NA & NA & NA & 4.00 \\
\hline 4-METHYL-2-PENTANONE & ma & NA & HA & NA & $u$ & 1.00 & 10.04 & NA \\
\hline RCE TOWE & NA & NA & MA & NA & NA & NA & 10.04 & NA \\
\hline ACROLEIN & $u$ & u & $u$ & U & NA & NA & $100.0 \mathrm{~s}$ & MA \\
\hline RCRYLOWITRILE & $u$ & $u$ & $u$ & $u$ & NA & NA & 100.00 & NA \\
\hline BENZENE & $u$ & $u$ & $u$ & $u$ & $u$ & $9.0 \mathrm{y}$ & 5.00 & $4.0 \mathrm{~J}$ \\
\hline BROMOBENZENE & HA & KA & NA & NA & MA & NA & $N A$ & 4.00 \\
\hline EROMOCHLOROME THAHE & NA & NA & in & HA & NA & NA & NA & 4.010 \\
\hline GROMOFORM & $u$ & u & $u$ & U & $u$ & 1.00 & 5. & $4.0 \mathrm{~J}$ \\
\hline CARBOW DISULFIDE & HA & kn & NA & ha & u & 1.00 & $5.0 \mathrm{~s}$ & NA \\
\hline CARBOW TETRACHLORIDE & $u$ & u & $u$ & $u$ & $u$ & 1.00 & $5.0 \mathrm{~J}$ & 4.00 \\
\hline LHLOROBENZENE & u & $u$ & $u$ & $u$ & $u$ & 1.04 & 5.00 & $4.8 \mathrm{~J}$ \\
\hline CHLORDO IBROMOMETHANE & $\mathbf{u}$ & $u$ & $u$ & $u$ & $u$ & 1.04 & $5.0 \mathrm{~J}$ & 4.04 \\
\hline CHLOROETHANE & u & $u$ & $u$ & $u$ & $u$ & 1.00 & $10.0 \mathrm{~J}$ & $4.0 \%$ \\
\hline CHLOROETHENE & $u$ & $u$ & $u$ & $u$ & $u$ & $1.0 . \mathrm{J}$ & $10.0 \mathrm{~s}$ & 4.00 \\
\hline CHLOROFORM & $u$ & $u$ & 6.7 & $u$ & $u$ & 1.00 & $5.0 \mathrm{~J}$ & 4. \\
\hline CIS-1,2-DICHLOROETHENE & NA & kA & WA & MA & HA & HA & NA & $4.0 \mathrm{~J}$ \\
\hline CIS-1,3-DICHLOROPROPENE & $u$ & u & $u$ & $u$ & $u$ & 1.00 & $5.0 \mathrm{U}$ & NA \\
\hline DIEROMOMETHAKE & MA & NA & HA & HA & HA & NA & NA & $4.0 \mathrm{~J}$ \\
\hline DICHLOROSROMOMETHANE & $u$ & U & $u$ & $u$ & $u$ & $1.0 \mathrm{~J}$ & $5.0 \mathrm{JJ}$ & 4.04 \\
\hline DICHLOROOIFLUOROMET THAHE & $u$ & $u$ & $u$ & $u$ & NA & NA & HA & $8.0 \mathrm{~J}$ \\
\hline ETHILBEKZENE & $u$ & $u$ & $u$ & $u$ & $u$ & $1.0 \mathrm{~s}$ & 5.00 & 4.04 \\
\hline
\end{tabular}


KANSAS CITY : SELECTED AHALYSIS FROM KC8S.046

Conet itwent

$\omega / L$

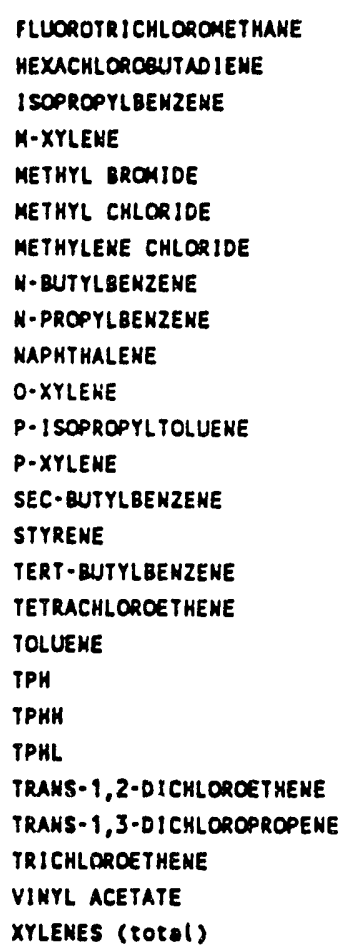

Semple Date

$08 / 04 / 86 \quad 02 / 04 / 87 \quad 10 / 39 / 88 \quad 02 / 11 / 89 \quad 07,18 / 90$

\begin{tabular}{|c|c|c|c|c|c|c|c|}
\hline$u$ & $\mathbf{U}$ & $\mathbf{U}$ & $\mathbf{U}$ & MA & NA & $10.0 U$ & $4.0 \mathrm{U}$ \\
\hline$M$ & $M$ & $M$ & $M A$ & MA & HA & NA & 5.00 \\
\hline$\mu$ & $\mu$ & $M$ & $M$ & NA & NA & NA & 4.00 \\
\hline MA & $M$ & $M$ & $m$ & NA & NA & NA & $4.0 \mathrm{~J}$ \\
\hline $\mathbf{U}$ & $\mathbf{U}$ & $\mathbf{U}$ & $\mathbf{U}$ & $\mathbf{U}$ & 1.00 & $10.0 \mathrm{U}$ & 4.00 \\
\hline $\mathbf{U}$ & $\mathbf{U}$ & $\mathbf{U}$ & $\mathbf{U}$ & $U$ & 1.00 & 10.00 & 4.00 \\
\hline 5.8 & 7.3 & $u$ & 6.1 & $U$ & 1.00 & 5.00 & 15.00 \\
\hline$M$ & $M$ & $M$ & $M A$ & NA & NA & NA & 4.00 \\
\hline KA & $\boldsymbol{M}$ & MA & $\boldsymbol{M}$ & NA & NA & NA & 4.00 \\
\hline NA & MA & MA & HA & HA & NA & NA & 14.00 \\
\hline NA & liA & 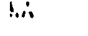 & HA & HA & his & $\therefore i$ & $\therefore .: J$ \\
\hline MA & MA & NA & MA & NA & HA & NA & 4.00 \\
\hline MA & NA & NA & NA & NA & NA & NA & 4.00 \\
\hline NA & NA & NA & NA & NA & NA & NA & 4.00 \\
\hline MA & MA & NA & NA & $U$ & 1.00 & 5.00 & 4.00 \\
\hline KA & KA & MA & NA & NA & HA & NA & 4.00 \\
\hline $\mathbf{U}$ & $\mathbf{U}$ & $U$ & $\mathbf{U}$ & $\mathbf{U}$ & 1.00 & 5.00 & 4.00 \\
\hline$U$ & $\mathbf{U}$ & $U$ & $\mathbf{U}$ & $U$ & $1.0 U$ & $5.0 \mathrm{U}$ & 4.00 \\
\hline KA & MA & NA & NA & MA & HA & $\mathrm{HA}$ & NA \\
\hline NA & $\boldsymbol{M}$ & NA & $M$ & MA & NA & HA & $N$ \\
\hline MA & NA & Wh & WA & NA & $N A$ & Wh & NA \\
\hline MA & MA & WA & NA & MA & NA & NA & 4.00 \\
\hline $\mathbf{u}$ & $\mathbf{U}$ & U & $\mathbf{U}$ & $\mathbf{U}$ & 1.00 & 5.00 & KA \\
\hline 4.3 & $\mathbf{U}$ & 76.2 & $\mathbf{U}$ & $\mathbf{U}$ & 1.00 & 5.00 & $4.0 \mathrm{U}$ \\
\hline KA & Mr & $M A$ & MA & $\mathbf{U}$ & 1.00 & $10.0 \mathrm{U}$ & NA \\
\hline$M$ & $M A$ & MA & NA & $\mathbf{U}$ & 1.00 & 5.00 & HA \\
\hline
\end{tabular}


KAHSAS CITY : SELECTED AHALYSIS FROM KCE5.044

Constituent

ug/L

$10 / 06 / 9$

$07 / 25 / 91$

$10 / 10 / 91$

\begin{tabular}{|c|c|c|c|}
\hline $1,1,1,2$ - TETRACHLOROETHANE & NA & NA & kA \\
\hline $9,1,1$ TRICHLOROETHAKE & 5.00 & 5.00 & 5.00 \\
\hline $1,1,2,2$ - TETRACHLOROE THANE & $5.0 \mathrm{~s}$ & $5.0 \mathrm{~s}$ & 5.04 \\
\hline $1,1,2-$ TRICHLOROETHANE & 5.00 & $5.0 \mathrm{~s}$ & 5.00 \\
\hline $1,1,2$-TRICHLOROTRIFLUOROETH & $5.0 \mathrm{~J}$ & $5.0 \mathrm{~J}$ & 5.00 \\
\hline 9,9 -DICHLOROETHANE & $5.0 . \mathrm{J}$ & $5.0 \mathrm{~s}$ & 5.00 \\
\hline 1,1-Dichloroet THENE & $5.0 \mathrm{~J}$ & $5.0 \mathrm{~J}$ & $5.0 \mathrm{~J}$ \\
\hline 1,1-DICHLOROPROPENE & iA & HA & kA \\
\hline 1,2,3-TRICHLOROBEHZENE & MA & NA & MA \\
\hline $1,2,3$-TRICHLOROPROPANE & NA & in & NA \\
\hline 1,2,3-TRIMETHYLBENZENE & kia & his & NA \\
\hline $1,2,4$-TRICHLOROBENZENE & Wh & in & NA \\
\hline 1,2-D1BROMO-3-CHLOROPROPANE & NA & iA & NA \\
\hline 1,2-DIGROMOET THANE & NA & HA & in \\
\hline 1,2-DICKLOROBEKZENE & NA & $5.0 \mathrm{~s}$ & 5.00 \\
\hline 1,2-DICHLOROETHANE & 5.04 & 5.00 & 5.00 \\
\hline 1,2-DICHLOROETKENE (IOTAl) & 5.00 & 5.00 & 24.0 \\
\hline 1,2-DICHLOROPROFANE & $5.0 \mathrm{~s}$ & 5.00 & $5.0 \mathrm{~J}$ \\
\hline 1,3,5-TRIMETUYLBENZENE & NA & hil & AA \\
\hline 1,3-DICHLOROBEKLENE & HA & 5.00 & 5.00 \\
\hline 1,3-DICHLOROPROPANE & MA & NA & NA \\
\hline 1,4-DICHLOROBENZENE & MA & 5.00 & 5.00 \\
\hline 2,2-DICHLOROPROPANE & MA & NA & MA \\
\hline 2-BUTANONE & 10.00 & 5.00 & 5.00 \\
\hline 2-CHLOROETHYLVINYL ETHER & NA & HA & $\mathrm{kA}$ \\
\hline 2-CHLOROTOLUENE & HA & HA & WA \\
\hline 2-HEXUAONE & 5.00 & $5.0 \mathrm{~J}$ & 5.00 \\
\hline 6-CHLOROTOLUENE & NA & Na & isA \\
\hline 4-METHYL-2-PENTAHOWE & 5.00 & 5.00 & 3.0u \\
\hline ACETONE & $10.0 \mathrm{~S}$ & 10.00 & 10.00 \\
\hline ACROLEIN & kA & NA & in \\
\hline ACRYLONITRILE & MA & NA & NA \\
\hline BENZENE & 5.04 & 5.00 & $5.0 \mathrm{~s}$ \\
\hline BRCMOBENZENE & ka & $N A$ & NA \\
\hline BROMOCHLOROMETHANE & NA & NR & KA \\
\hline BROMOFORM & 5.00 & 5.04 & $5.0 \mathrm{~J}$ \\
\hline CARBOW DISULFIDE & HA & 5.00 & $5.0 \mathrm{~J}$ \\
\hline CARBON TETRACHLORIDE & $5.0 \mathrm{~s}$ & 5.00 & 5.00 \\
\hline CHLOROBEHZENE & 5.04 & 5.00 & $5.0^{\prime} \mathrm{J}$ \\
\hline CHLORDO I BROMOMET KAHE & 5.00 & 5.04 & $5.00 \mathrm{~s}$ \\
\hline CHLOROETHRHE & $10.0 \mathrm{~J}$ & $10.0 \mathrm{~s}$ & 10.0.5 \\
\hline CHLOROE THENE & $10.0 \mathrm{~J}$ & 10.00 & $10.0 \mathrm{v}$ \\
\hline CHLOROFORM & $5.0 \mathrm{~s}$ & 5.00 & 5.00 \\
\hline CIS-1,2-DICHLOROETHENE & NA & NA & kA \\
\hline CIS-1,3-OICMLOROPROPENE & 5.00 & $5.0 \mathrm{~s}$ & 5.00 \\
\hline DIBROMOMETHANE & NA & NA & NA \\
\hline DICHL OROLROMOMETHANE & $5.0 \mathrm{~s}$ & 5.00 & 5.00 \\
\hline DICHLORDO I FLUOROME THANE & NA & KA & in \\
\hline ETHYLBENZENE & $5.0 \mathrm{~J}$ & 5.00 & 5.0 .5 \\
\hline
\end{tabular}


rUNSAS CITY : SELECTED AHALYSIS FROM KC85.024

ansifituent

$\omega / 6$

$10 / 06 / 90 \quad 07 / 25 / 91 \quad 10 / 10 / 91$

\begin{tabular}{|c|c|c|c|}
\hline FLUOROTRICHLORONETHANE & $m$ & 10.0 & $10 . \infty$ \\
\hline MEXUCNLOROQUTAD JEME & ma & $m$ & $\boldsymbol{m}$ \\
\hline ISDPROP YLBENZEHE & ma & $m$ & $m$ \\
\hline M-XYLEME & $m$ & MA & $m$ \\
\hline KETHYL BRONIDE & 5.00 & 10.00 & 10.00 \\
\hline METHYL CKLORIDE & $10.0 \mathrm{U}$ & 1c.os & 10.0 \\
\hline METHYLENE CHLORIOE & 5.0 & 5.20 & s.a \\
\hline M-QUTYLLEENZENE & Ma & MA & Ma \\
\hline N-PROPYLBEXZENE & KA & MA & MA \\
\hline KAPHTMALENE & ma & MA & NA \\
\hline 0.XYLENE & MA & KA & KA \\
\hline P-I SOPROPYLIOLLUENE & NA & MA & MA \\
\hline P·XYLENE & MA & MA & WA \\
\hline SEC-BUTYLBENZENE & MA & $m$ & $m$ \\
\hline STYREAE & $5.0 \mathrm{~d}$ & 5.00 & s.w \\
\hline TERT-DUTYLBEN2ENE & MA & MA & MA \\
\hline TETRACHLOROETHENE & 5.00 & 5.01 & 5.0 \\
\hline TOLUENE & 3.0 & 5.00 & 5.00 \\
\hline TPH & MA & MA & WA \\
\hline TPHA & KA & $m$ & Ma \\
\hline TPHL & MA & $m$ & MA \\
\hline TRAMS-1,2-DICHLOROETHENE & KA & MA & KA \\
\hline TRAHS-1,3-OICHLCROPROPENE & 5.00 & 5.00 & $5 . w$ \\
\hline TRICHLOROETKEKE & 5.00 & 5.00 & s.os \\
\hline VIMYL ACETATE & MA & ma & $\mathbf{m a}$ \\
\hline XYLENES (\$Otal) & 15.00 & 5.00 & s.w \\
\hline
\end{tabular}

Sumple Date 
KAHSAS CITY : SELECTED AHALYSIS FROM KCE8.094.L

Constituent

ug/L

$04 / 26 / 89$

Sample Date

\begin{tabular}{|c|c|c|c|c|c|c|c|c|}
\hline 1,1,1,2-TETRACHLOROETHAHE & HA & NA & HA & NA & NA & 4.00 & 4.00 & NA \\
\hline $1,1,1 \cdot T R I$ CHLOROETHANE & $1.0 \mathrm{~J}$ & $5.0 \mathrm{U}$ & 5.04 & 5.00 & 5.00 & $4.0 \mathrm{~s}$ & 4.00 & $5.0 \mathrm{~J}$ \\
\hline $1,1,2,2$-TETRLCHLOROETHAKE & 1.00 & 5.00 & 5. Q O & 5.03 & 5.00 & 4.00 & 4.00 & 5.00 \\
\hline $1,1,2 \cdot T R I C H L O R O E T$ THAE & 1.0 .0 & 5.00 & 5.01 & 5.00 & 5.00 & 4.00 & 4.00 & $5.0 \mathrm{~J}$ \\
\hline 1,1,2-TRICHLOROTRIFLUOROETH & iA & NA & HA & KA & NA & HA & iA & $5.0 \mathrm{U}$ \\
\hline 1,1-DICHLOROETKLKE & 1.00 & 5.00 & $5.0 \mathrm{~s}$ & 5.00 & 5.00 & $4.0 \mathrm{~J}$ & $4.0 \mathrm{U}$ & $5.0 \mathrm{U}$ \\
\hline 1, १-DICHLOROETHEHE & $1.0 \mathrm{~J}$ & 5.00 & 5.00 & 5.00 & $5.0 \mathrm{~J}$ & $7.0 \mathrm{~J}$ & $7.0 \mathrm{~J}$ & $5.0 \mathrm{U}$ \\
\hline 1,1-DICHLOROPROPEKE & hiA & NA & NA & hiA & NA & $4.0 \mathrm{~J}$ & 4.00 & NA \\
\hline 1,2,3-TRICHLOROBENZENE & ina & kA & MA & HA & His & $5.0 \mathrm{~J}$ & 5.00 & NA \\
\hline $1,2,3 \cdot T R I C H L O R O P R O P A N E$ & NA & NA & NA & HA & NA & 4.00 & 4.00 & N'A \\
\hline $1,2,3 \cdot(R)$ METHYLEENZENE & NA & hA & NA & hA & in & $5.0 \mathrm{~J}$ & 5.00 & NA \\
\hline 1,2,4-TRICHLOROSEK'ZENE & NA & NA & NA & SA & NA & $5.0 \mathrm{~J}$ & 5.00 & NA \\
\hline 1,2-01BROMO-3-CHLOROPROPAKE & NA & NA & HiA & NA & NA & $4.0 \mathrm{~J}$ & 4.00 & NA \\
\hline 1,2-015ROMOETHANE & KA & ka & NA & HA & hiA & 4.00 & $4.0 \mathrm{~J}$ & NA \\
\hline 9,2-DICHLOROSEKZENE & NA & KA & HA & NA & NA & $4.0 \mathrm{~J}$ & 4.00 & KiA \\
\hline 1,2-DICHLOROETHAHE & 1.00 & 5.00 & 5.00 & $5.0 U$ & 5.00 & $4.0 \mathrm{~s}$ & 4.00 & 5.04 \\
\hline 1,2-DICHLOROETHENE (total) & 1.00 & $5.0 \mathrm{~s}$ & 5.00 & 5.00 & 5.00 & NA & ia & 5.00 \\
\hline 1,2-DJCHLOROPROPANE & $1.0 \mathrm{~J}$ & 5.00 & $5.0 \mathrm{U}$ & $5.0 U$ & 5.00 & 4.00 & 4.00 & 5.00 \\
\hline 1,3,5-TRIMETHYLBER'ZENE & NA & NA & NA & MA & KA & $5.0 \mathrm{~J}$ & 5.00 & NA \\
\hline 1,3-DICHLOROBENZENE & NA & NA & HA & hA & NA & 4.00 & 4.00 & NA \\
\hline 1,3-OICHLOROPROPANE & HA & HA & NA & NA & HA & 4.015 & 4.00 & NA \\
\hline 1,4-DICHLOROSENZENE & NA & MA & NA & NA & HA & 4.00 & 4.00 & NA \\
\hline 2,2-DICHLOROPROPANE & NA & NA & kA & NA & NA & 4.00 & 4.00 & NA \\
\hline 2-BUTANONE & 1.00 & 10.00 & 10.00 & 10.00 & 50.0 & NA & NA & 10.00 \\
\hline 2-CHLOROETHYLVIKYL ETHER & 1.00 & 10.00 & $10.0 \mathrm{~s}$ & 90.00 & NA & SA & NA & MA \\
\hline 2-CHLOROTOLUENE & NA & NA & MA & NA & NA & 4.00 & $4.0 \mathrm{~J}$ & NA \\
\hline 2-HEXANONE & 1.04 & 10.00 & $10.0 \mathrm{~s}$ & 10.00 & 10.01 & HA & NA & 5.00 \\
\hline 6-CHLOROTOLUENE & MA & NA & Na & NA & NA & $4.0 \mathrm{~s}$ & 4.00 & NA \\
\hline 4-METHYL-2-PENTANONE & 1.00 & 10.01 & 10.04 & $10.0 \mathrm{~J}$ & 20.0 & NA & KA & $5.0 \mathrm{U}$ \\
\hline ACE TOWE & NA & 10.00 & 10.00 & 10.05 & $10.0 \mathrm{~s}$ & NA & NA & 10.00 \\
\hline ACROLEIH & NA & 100.00 & 100.00 & 100.00 & $N A$ & HA & NA & NA \\
\hline ACRYLONITRILE & NA & 100.00 & 100.00 & 100.00 & NA & NA & NA & NA \\
\hline BENZEHE & $1.0 \mathrm{~J}$ & 5.00 & 5.00 & 5.00 & 5.00 & 4.00 & $4.0 \mathrm{U}$ & $5.0 U$ \\
\hline BROMOSEK2ENE & HA & NA & NA: & NA & NA & 4.00 & 4.00 & NA \\
\hline BROHOCHLOROMETHANE & NA & NA & NA & NA & NA & 4.00 & 4.00 & NA \\
\hline SROMOF ORM & $1.0 \mathrm{~J}$ & $5.0 \mathrm{U}$ & 5.04 & 5.00 & 5.00 & 4.00 & 4.00 & $5.0 \mathrm{U}$ \\
\hline CARBOW DISULFIDE & 1.05 & $5.0 \mathrm{~s}$ & 5.04 & 5.00 & $5.0 \mathrm{~J}$ & NA & NA & N'A \\
\hline CARBOW TETRACHLORIDE & $1.0 \mathrm{~J}$ & $5.0 \mathrm{~s}$ & $5.0 \mathrm{U}$ & 5.00 & 5.00 & 4.00 & $4.0 \mathrm{~J}$ & 5.00 \\
\hline CHLOROBENZENE & $1.0 \mathrm{~J}$ & 5.00 & 5.00 & 5.04 & $5.0 \mathrm{~s}$ & 4.00 & 4.00 & $5.0 \mathrm{u}$ \\
\hline CHLORDOIBROAOMETHANE & $1.0 \mathrm{~J}$ & 5.00 & 5.00 & 5.00 & 5.00 & 4.00 & 4.00 & $5.0 \mathrm{~J}$ \\
\hline ELLOROETHANE & 1.00 & 10.00 & $10.0 \mathrm{~s}$ & 10.00 & 10.00 & $4.0 \mathrm{~s}$ & $4.0 \mathrm{U}$ & 10.00 \\
\hline SHLOROETHENE & 1.00 & 10.04 & 10.00 & $10.0 \mathrm{~J}$ & 10.00 & $4.0 \mathrm{~s}$ & 4.00 & 10.00 \\
\hline :HLOROFORM & $1.0 \mathrm{~J}$ & 5.00 & 5.00 & 5.00 & 5.00 & 4.00 & 4.00 & 5.04 \\
\hline :IS-1,2-DICHLOROETHENE & in & hil & HA & hA & HA & $4.0 \mathrm{U}$ & 4.00 & NA \\
\hline :I5-1,3-DICHLOROPROPENE & $1.0 \mathrm{~J}$ & 5.00 & 5.00 & $5.0 \mathrm{~J}$ & $5.0 \mathrm{~J}$ & NA & MA & $5.0 \mathrm{~J}$ \\
\hline IBROMONE T HAKE & WA & NA & MA & his & HA & 4.015 & 4.00 & NA \\
\hline ICHLOROBROMOMETHANE & $1.0 \mathrm{~J}$ & 5.00 & $5.0 \mathrm{~J}$ & $5.0 \mathrm{~J}$ & $5.0 \mathrm{u}$ & 4.00 & 4.00 & $5.0 \mathrm{~J}$ \\
\hline ICHLOROO I FLUOROME T HAKE & NA & NA & NA & NA & NA & $8.0 \mathrm{U}$ & 8.00 & NA \\
\hline THYLBEHZENE & 1.00 & $5.0 \mathrm{~J}$ & 5.00 & $5.0 \mathrm{~J}$ & 5.015 & 4.0.J & $4.0 \mathrm{~J}$ & $5.0 \mathrm{~J}$ \\
\hline
\end{tabular}


KANSAS CITY : SELECYED AMALYSIS FROM KCB8-094-L

constituent

- cos

$\begin{array}{lllllll}10 / 31 / 88 & 02 / 91 / 80 \quad 06 / 26 / 89 & 07 / 26 / 89 & 10 / 26 / 89 & 01 / 30 / 90 & 04 / 24 / 90 & 10 / 06 / 90\end{array}$

Serple Date

\begin{tabular}{|c|c|c|c|c|c|c|c|c|}
\hline FLUOROTRICHL OROAETHANE & $M$ & 10.00 & 10.00 & $10 . \boldsymbol{\alpha}$ & $M$ & 4.00 & 6.00 & NA \\
\hline HEXACHLOROQUT ADIENE & M & MA & $\boldsymbol{M}$ & $k 4$ & $M$ & $5 . \infty$ & 5.00 & NA \\
\hline ISOPROPYLBENZENE & $M$ & MA & $m$ & $\$ 8$ & $\mathbf{m}$ & 4.00 & 4.00 & NA \\
\hline M.XYLENE & 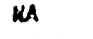 & $M A$ & $M$ & HA & $M$ & 6.0 & 4.00 & MA \\
\hline METKYL BROAIDE & 1.00 & 10.00 & $10.0 \mathrm{w}$ & rivow & 10.00 & 4.00 & 4.00 & 5.00 \\
\hline METKYL CHILORIDE & 1.00 & 10.01 & 10.00 & 10.00 & 10.0 & 4.00 & 4.00 & 10.00 \\
\hline RETHYLENE CHLORIDE & 1.0 & $5.0 \mathrm{~J}$ & 5.00 & 5.0 & $5.0 \omega$ & $15 . \infty$ & 15.04 & $5.0 u$ \\
\hline N-QUTYLBENZENE & $M$ & $\boldsymbol{M}$ & $M$ & $M$ & M & $4 . \infty$ & 4.00 & NA \\
\hline M-PROPYLBENZENE & MA & MA & $M$ & $\mathbf{x A}$ & $\boldsymbol{\mu}$ & 4.00 & $4.0 U$ & HA \\
\hline NAPHTHALENE & NA & KA & KA & in & NA & 16.00 & 14.04 & HA \\
\hline O-XYLEKE & NA & hi. & KA & $\times 4$ & in & 4.00 & $\because \cdots$ & $\because$ \\
\hline P. I SOPROPYLTOLUEKE & NA & NA & NA & KA & his & 6.00 & $\because 1$ & $\cdots$ \\
\hline P.XYLENE & NA & MA & MA & NA & NA & 4.00 & 4.00 & hA \\
\hline SEC-SUTYLLENZENE & NA & MA & KA & KA & NA & 4.00 & 4.00 & HA \\
\hline STYRENE & $1 . \infty$ & 5.01 & 5.00 & 5.00 & 5.00 & 4.00 & 4.00 & 5.00 \\
\hline TERT-BUTYLBENZENE & MA & NA & $\boldsymbol{M}$ & $m$ & NA & 4.00 & 4.00 & HA \\
\hline TETRACHLOROETHEHE & 1.00 & 5.05 & 5.00 & 5.00 & 5.00 & 4.01 & 4.00 & 5.04 \\
\hline TOLUENE & 1.00 & $5 . \infty$ & 5.00 & 5.00 & $5 . \infty$ & 6.00 & 4.00 & 5.00 \\
\hline TPH & MA & MA & M & WA & NA & NA & MA & NA \\
\hline PHK & MA & MA & MA & NA & NA & NA & NA & MA \\
\hline IPNL & $\boldsymbol{M A}$ & $\mathbf{M A}$ & $\boldsymbol{M A}$ & NA & NA & NA & MA & NA \\
\hline TRANS-1,2-DICHLOROETHENE & MA & MA & MA & NA & NA & 4.00 & 4.00 & NA \\
\hline TRANS-1,3-DICHLOROPROPEHE & 1.00 & 5.00 & 5.00 & 5.00 & 5.00 & NA & NA & $5.0 \mathrm{U}$ \\
\hline TRICHLOROETHENE & 1.00 & 5.00 & 5.00 & 5.00 & 5.04 & 4.00 & 4.00 & $5.0 \mathrm{U}$ \\
\hline VINYL ACETATE & 1.00 & 10.0 & 10.00 & 10.00 & 10.00 & NA & NA & HA \\
\hline XYLENES (total) & 1.00 & 5.00 & $5 . \alpha$ & 5.00 & 5.00 & MA & HA & 15.00 \\
\hline
\end{tabular}


KAKSAS CITY : SELECTED AKALYSIS FROM KC83.094-L

Constituent

$01 / 16 / 91 \quad 04 / 25 / 99 \quad 07 / 20 / 91 \quad 10 / 91 / 91$

\begin{tabular}{|c|c|c|c|c|}
\hline $1,1,1,2$-TETRACHLOROETHANE & NA & NA & HA & HA \\
\hline $1,1,1$-TRICHLOROETHANE & $5.0 \mathrm{~J}$ & 5.00 & $5.0 \mathrm{~J}$ & 5.00 \\
\hline $1,1,2,2$ - TETRACHLOROETHANE & $5.0 \mathrm{~s}$ & 5.00 & $5.0 \mathrm{~J}$ & $5.0 \mathrm{~J}$ \\
\hline $1,1,2-T R I C H L O R O E T H A N E$ & $5.0 \mathrm{j}$ & $5.0 \mathrm{~J}$ & 5.00 & 5.00 \\
\hline 1, 1,2-TRICHLOROTRIFLUOROETH & $5.0 \mathrm{U}$ & 5.00 & 5.00 & 5.00 \\
\hline 1,1-DICHLOROETHANE & 5.00 & 5.00 & $5.0 \mathrm{~s}$ & 5.04 \\
\hline $1,1 \cdot$ DICHLOROETHENE & $5.0 \mathrm{~d}$ & $5.0 \mathrm{~J}$ & 5.00 & $5.0 i$ \\
\hline $1,9 \cdot$ DICHLOROFROPENE & NA & NA & NA & NA \\
\hline $1,2,3-T R / C H L O R O B E N Z E N E$ & hiA & Wh & HA & NA \\
\hline $1,2,3$-TRICHLOROPROSANE & NA & $A A$ & NA & NA \\
\hline $1,2,3-T R I$ IHETHYLBENZENE & h'A & NA & h'A & NA \\
\hline $1,2,4$-TRICHLOROSERZENE & NA & NA & NA & hA \\
\hline 1,2-DIBROMD-3-CHLOROPROPAKE & NA & AA & NA & HA \\
\hline 1,2-01BROMOETHANE & HA & NA & NA & NA \\
\hline 1,2-DICHLOROEERZEKE & NA & NA & 5.00 & 5.00 \\
\hline 1,2-DICHLOROEI HANE & 5.00 & 5.00 & 5.00 & 5.00 \\
\hline 1,2-DICHLOROETHENE (tOTAl) & 5.00 & 5.00 & 5.00 & $5.0 \mathrm{~s}$ \\
\hline 1,2-DICHLOROPROPANE & $5 . \omega$ & 5.00 & 5.00 & 5.00 \\
\hline 1,3,5-TRIMETHYLBEHZENE & NA & NA & NA & NA \\
\hline 1,3-DICKLOROBENZENE & NA & NA & 5.00 & $5.0 \mathrm{~J}$ \\
\hline 1,3-DICHLOROPROPANE & NA & NA & MA & NA \\
\hline 1,4-DICHLOROBENZENE & HA & NA & 5.00 & 5.04 \\
\hline$?, 2$-DICHLOROPROPAHE & NA & NA & NA & NA \\
\hline -BUTANONE & 5.00 & 5.00 & 5.00 & 5.00 \\
\hline 2-CKLOROETHYLVINYL ETKER & HA & NA & NA & NA \\
\hline 2-CHLOROTOLUENE & NA & NA & NA & NA \\
\hline 2-KEXANONE & 5.00 & $5.0 \mathrm{U}$ & 5.00 & $5.0 \mathrm{~s}$ \\
\hline 4-CHL OROTOLUENE & NA & NA & NA & NA \\
\hline 4-KETHYL-2-PENTANONE & 5.00 & 5.00 & 5.00 & 5.00 \\
\hline ACETONE & 10.04 & $10.0 \mathrm{U}$ & 10.05 & 10.00 \\
\hline ACROLEIN & MA & NA & KA & NA \\
\hline ACRYLONITRILE & NA & NA & NA & HA \\
\hline BENZENE & $5.0 \mathrm{U}$ & 5.00 & 5.00 & 5.00 \\
\hline BROMOBEN2ENE & NA & HA & NA & NA \\
\hline BROMOCHL OROMETHANE & NA & HA & NA & NA \\
\hline BROMOFORM & $5.0 \mathrm{~s}$ & 5.00 & 5.00 & 5.00 \\
\hline CARBON DISULFIDE & NA & NA & 5.00 & 5.00 \\
\hline CARBON TETRACHLORIDE & 5.00 & 5.00 & 5.00 & 5.00 \\
\hline CHLOROBENZENE & 5.04 & 5.00 & 5.00 & 5.00 \\
\hline CHLORCO I GROMOME T HAKE & 5.08 & 5.00 & 5.00 & $5.0 \mathrm{~s}$ \\
\hline CHLOROETHANE & 10.0U & $10.0 U$ & 10.00 & $10.0 \mathrm{U}$ \\
\hline CHLOROETHENE & $10.0 \mathrm{~J}$ & 10.00 & 10.04 & $10.0 \mathrm{~J}$ \\
\hline CHLOROF ORM & 5.0 & 5.00 & $5.0 \mathrm{~J}$ & 5.04 \\
\hline CIS-1,2-DICHLOROE THENE & NA & hA & WA & MA \\
\hline CIS-1,3-DICHLOROPROPENE & 5.01 & 5.04 & 5.00 & 5.00 \\
\hline DIGROMOMETKLNE & hA & BA & HA & NA \\
\hline DICHLOROSROMOMETHRHE & 5.005 & 5.00 & 5.00 & 5.00 \\
\hline DICHLORCOIFLUOROMETHANE & NA & ha & NA & NA \\
\hline ETKYLBEH'ZENE & 5.00 & $5.0 \mathrm{~J}$ & $5.0 \mathrm{~J}$ & $5.0 \mathrm{~J}$ \\
\hline
\end{tabular}


RANSAS CITY : SELECTED AKALYSIS IROA KCB8.096-L

Seaple Date

Const it tuent

$u=/ 6$

$01 / 16 / 91 \quad 04 / 25 / 91 \quad 07 / 20 / 91 \quad 10 / 11 / 91$

\begin{tabular}{|c|c|c|c|c|}
\hline PLLOROTRICKLORONET THANE & $m$ & 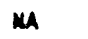 & 10.00 & 10.01 \\
\hline MEXUCHL OROQUTNOIENE & $m$ & $M$ & $\mu$ & $m$ \\
\hline I SOPRCPYLBENZENE & $\mu$ & $m$ & $\mu$ & $m$ \\
\hline M-XYLEME & $\mu$ & $\mu$ & $m$ & $m$ \\
\hline METHYL BROMIDE & $10 . \infty$ & 10.00 & 10.00 & $10.0 \mathrm{~d}$ \\
\hline METHYL CHLORIDE & $10 . \infty$ & 10.00 & 10.00 & 10.00 \\
\hline METHYLEME CKLORIDE & 5.00 & $5.0 \mathrm{~s}$ & 5.0 & s.as \\
\hline N-EUTYLBEMZEME & $m$ & 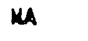 & $m$ & $m$ \\
\hline N-PROPYLBEKZEKE & Ma & $m$ & Mu & $m$ \\
\hline RAPHTHALEKE & MA & $x A$ & MA & kA \\
\hline $0 \cdot \ddot{X}, L E \because \Xi$ & $\therefore:$ & $\therefore$. & $\therefore$ & MA \\
\hline P. I SOPROPYLTOLUENE & MA & $k \mu$ & M & NA \\
\hline P·XYLENE & MA & HA & $m$ & HA \\
\hline SEC-BUTYLBENZENE & MA & MA & KA & HA \\
\hline SITRENE & 5.w & $5.0 \mathrm{~J}$ & 5.00 & 5.00 \\
\hline TERT-BUTYLBENZENE & MA & $M A$ & $M A$ & MA \\
\hline TETRACHLOROETHEKE & 5.00 & 5.01 & $5 . \alpha$ & $5 . \infty$ \\
\hline TOLUENE & 5.01 & 5.00 & 5.0 & s.ou \\
\hline TPH & MA & Ma & $m$ & $m$ \\
\hline PHK & $\boldsymbol{\mu}$ & ka & $M$ & MA \\
\hline TPHL & $m$ & MA & $\mathbf{m a}$ & MA \\
\hline TRAKS-1,2-01CHLOROE THENE & KA & MA & MA & $m \boldsymbol{A}$ \\
\hline TRANS-1,3-DICHLCROPROPENE & 5.0 & 5.04 & 5.0 & 5.00 \\
\hline TRICALOROETHEME & 5.0U & 5.00 & 5.00 & 5.00 \\
\hline VINYL ACETATE & MA & MA & MA & MA \\
\hline XYLENES (total) & $5 . \infty$ & $5 . \alpha$ & $5 . \omega$ & $5 . \infty$ \\
\hline
\end{tabular}




\section{$C-27$}

RALSAS CITY : SELECTED ALALYSIS FROM KCBS-094-U

Constitwent

us/l

$04 / 25 / 90$

$07 / 19 / 90$

$10 / 06 / 90$

\begin{tabular}{|c|c|c|c|}
\hline 1,1,1,2-TETRACHLOROETHAHE & 4.00 & 4.00 & HA \\
\hline $1,1,1-1 R I$ CHLOROEYKKKE & 4.00 & $4.0 \mathrm{~J}$ & $5.0 \mathrm{~J}$ \\
\hline $1,1,2,2$ - TETRACHL OROE TKAKE & $4.0 \mathrm{~J}$ & 4.00 & 5.00 \\
\hline 1,1,2-TRICKLOKOETHLKE & $4.0 \mathrm{~J}$ & $4.0 \mathrm{~J}$ & $5.0 \mathrm{~J}$ \\
\hline 1,1,2-IRICHLOROIRIFLLUOROETH & SA & NA & 5.04 \\
\hline 1,9-DICHLOROETHAHE & $4.0 \mathrm{~J}$ & 4.00 & 5.04 \\
\hline $1,9 \cdot$ DICHLOROE THENE & $7.0 \mathrm{~J}$ & 7.00 & $5.0 \mathrm{~J}$ \\
\hline $1,1.01$ CHLOROPROPENE & 4.00 & 4.00 & NA \\
\hline $1,2,3-T R I C H L O R O B E K ' 2 E$ HE & $5.0 \mathrm{~J}$ & $5.0 \mathrm{~s}$ & HA \\
\hline $1,2,3$-TRICHLOROPROPANE & 4.0 .5 & $4.0 \mathrm{~J}$ & HA \\
\hline $1,2,3 \cdot T R I M E T H Y L G E A Z E N E$ & $5.0 \mathrm{~J}$ & $5.0 \mathrm{~J}$ & HA \\
\hline 1,2,4-TRICHLOROBEKIZENE & 5.00 & $5.0 \mathrm{~s}$ & NA \\
\hline 1,2-OIGROMO-3-CHLOROPRDPANE & $4.0 \mathrm{~J}$ & $4.0 \mathrm{~J}$ & KA \\
\hline 1,2.DIBROMOETHANE & 4.00 & 4.00 & HA \\
\hline 1,2-DICHLOROGEK'ZEKE & $4.0 \mathrm{~J}$ & 4.00 & NA \\
\hline 1,2-DICHLOROETKLHE & 4.05 & $4.0 \mathrm{~J}$ & 5.00 \\
\hline 1,2-DICHLOROETHENE (total) & NA & NA & $5.0 \mathrm{U}$ \\
\hline 1,2-DICHLOROPROPAHE & 4.00 & 4.00 & $5.0 \mathrm{v}$ \\
\hline $1,3,5$ - TRIMETHYLBERZENE & $5.0 \mathrm{~J}$ & $5.0 \mathrm{~s}$ & NA \\
\hline 1,3-DICHLOROSEN'ZENE & 4.00 & 4.00 & NA \\
\hline 1,3-DICKLOROPROPAKE & $4.0 \mathrm{~s}$ & 4.00 & NA \\
\hline 1,4-DIT.HLOROSEKLEHE & 4.00 & 4.00 & NA \\
\hline 2,2-DICHLOROPROPANE & 4.04 & 4.00 & NA \\
\hline 2-BUTANONE & NA & NA & 10.00 \\
\hline 2-CHLOROETHYLVIKYL ETHER & NA & NA & NA \\
\hline 2-CHLOROTOLUENE & 4.00 & 4.00 & NA \\
\hline 2- HEXANONE & HA & NA & 5.00 \\
\hline 4-CHLOROTOLUENE & 4.00 & 4.ov & NA \\
\hline 4-METHYL-2-PENTANONE & NA & NA & 5.00 \\
\hline RCETONE & NA & NA & 19.0 \\
\hline ACROLEIN & NA & NA & NA \\
\hline ACRYLOWITRILE & KA & NA & KA \\
\hline BENZENE & 4.00 & $4.0 \mathrm{~J}$ & $5.0 \mathrm{~J}$ \\
\hline BROMOBENZENE & 4.00 & 4.00 & HA \\
\hline BROMOCHLOROMET THAHE & 4.015 & 4.00 & HA \\
\hline BROMOFORH & 4.00 & 4.00 & 5.00 \\
\hline CARBOW DISULFIDE & NA & NA & NA \\
\hline CARBOM TETRACHLORIDE & 4.00 & 4.00 & 5.00 \\
\hline CHLOROSEL'ZENE & 4.00 & 4.04 & $5.0 \mathrm{~J}$ \\
\hline CHLORDO IBROMOME THAHE & 4.00 & 4.00 & 5.01 \\
\hline CHLOROETHALE & $4.0 \mathrm{~J}$ & 4.015 & 10.00 \\
\hline CHLOROETHEKE & 4.0 .5 & 4.0 .5 & 10.00 \\
\hline CHLOROFORM & $4.0 \mathrm{~s}$ & $6.0 \mathrm{~J}$ & 5.00 \\
\hline CIS-1,2-DICHLOROETKENE & $4.0 \mathrm{~J}$ & $4.0 \mathrm{~s}$ & NA \\
\hline CIS-9,3-DICHLOROPROPEKE & NA & NA & 5.00 \\
\hline DISROMOMETKRHE & 4.00 & 4.00 & NA \\
\hline DICHLOROSROMOMETHANE & 4.03 & 4.00 & 5.00 \\
\hline DICHLOROO I FLUSROOME T HAHE & $8.0 \mathrm{~s}$ & $8.0 \mathrm{~J}$ & hi \\
\hline ETHYLEENZENE & $4.0 \mathrm{~J}$ & $4.0 \mathrm{~J}$ & $5.0 \mathrm{u}$ \\
\hline
\end{tabular}

Sample Dare 
KANSAS CITY : SELECTED AMALYSIS FROM KCB8.096-U

Sample Date

constif twent

us/t

$04 / 25 / 90 \quad 07 / 19 / 90 \quad 10 / 06 / 90$

\begin{tabular}{|c|c|c|c|}
\hline FLUOROIRICHLOROKETHUNE & 6.00 & 6.00 & $m$ \\
\hline MEXUCHLOROQUTNDIENE & 5.00 & s.w & $m$ \\
\hline ISOPROPYLBENZENE & 4.00 & 4.00 & $m$ \\
\hline N-XYLEXE & 6.00 & 4.W & $M$ \\
\hline METHYL BROMIDE & $4 . w$ & 6.00 & $5 . a$ \\
\hline RETHYL CMLORIDE & 6.00 & 4.00 & $10 . a$ \\
\hline METHYLENE CHLORIDE & 15.00 & 15.00 & $5 . a$ \\
\hline W-BUTYLLEENZENE & 6.00 & 4.00 & $M$ \\
\hline N-PROPYLBEKZENE & h.os & 4.00 & M \\
\hline NAPHT HALENE & 14.00 & 16.00 & kA \\
\hline O.XYLELEE & ' j & $\cdot$ & $\therefore$. \\
\hline P. I SOPROPYLTOLUELEE & 4.0 & 4.00 & kik \\
\hline P-XYLENE & $6 . \infty$ & 4.00 & NA \\
\hline SEC-BUTYLBENZENE & 4.00 & 6.00 & Ma \\
\hline STYREME & 6.00 & 4.0u & $5 . \alpha$ \\
\hline TERT-BUTYLBENZENE & 4.00 & 4.00 & $\mathrm{ma}$ \\
\hline TETRACHLOROETHENE & 6.00 & 4.00 & $5 . a$ \\
\hline TOLUENE & 4.00 & 4.0u & $5 . a$ \\
\hline TPH & HA & MA & MA \\
\hline -PHH & $m$ & M.4 & kA \\
\hline ,PHL & MA & MA & $\boldsymbol{m}$ \\
\hline TRANS-1,2-DICHLOROET HENE & 4.00 & 4.W & NA \\
\hline TRANS-1,3-DICHLOROPROPENE & NA & MA & s.a \\
\hline TRICHLOROETHENE & 6.00 & 4.00 & $5 . a$ \\
\hline VIKYL ACETATE & MA & MA & MA \\
\hline XYLENES (totol) & MA & ma & $15 . a$ \\
\hline
\end{tabular}




\section{C -29}

KANSAS CITY : SELECTED AMALYSIS FROM KC99-158-L

ionstituent

us/l

1,1,1,2-TETRACHLOROETHAME

$1,1,1$ - IRICHLOROE THANE

$1,1,2,2$ - TETRACHLOROETHANE

1,1,2-TRICHLOROETHANE

$1,1,2$ - TRICHLOROTRIFLUOROETH

1,1-DICHLOROE THANE

1,1 -OICHLOROETHENE

1,1-DICHLOROPROPEME

$1,2,3$-TRICHLOROBERZENE

$1,2,3$-TRICHLOROPROPAKE

$1,2,3$-TRIMETHYLBER'ZENE

$1,2,4$-TRICHL OROBENZENE

1,2 -DIBROMH-3-CHLOROPROPANE

$1,2 \cdot 01$ BROMOETHANE

1,2 -DICKL OROBENZENE

1,2 - DICHLOROE THAME

1,2-01CHLOROETHENE (tOtDl)

1,2-DICKLOROPROPANE

$1,3,5$-TRIMETHYLBEKZENE

,3-DICHLOROBENZENE

1,3 -DICHLOROPROPANE

1,4-DICHL OROBENZENE

2,2-DICHLOROPROPANE

2-BUTAMONE

2-CHLOROE THYLVINYL ETHER

2- CHLOROTOLUENE

2- HEXAMONE

4-CHLOROTOLUENE

6-METHYL-2-PEMTAMONE

ACETONE

ACROLEIN

ACRYLOWITRILE

GEMZENE

OROMOBE MZENE

BROWOCHL OR OME THAME

BrowoForM

CARBOW DISULFIDE

CAREOW TETRACMLORIDE

CHLOROBENZENE

CHLCRCOIBROMOME THANE

CHLOROE THAME

CHLOROETHENE

CHLOROFORM

CIS-1,2-OICHLOROETHENE

CIS-1,3-DICHLOROPROPENE

IBRONOME THANE

DICHLOROBROMOME T HAME

DICHLORCO I FLLOROMETHANE

ETHYLBEKZEME
MA

5.00

5.00

5.0

5.01

5.0

5.00

MA

MA

MA

NA

MA

MA

MA

5.00

5.0

6.0

5.00

NA

5.00

MA

5.00

MA

s.or

MA

MA

s.ou

MA

5.00

10.00

MA

ua

5.00

MA

MA

5.0

5.00

s.0

5.00

5.00

10.00

10.00

s.ou

$\mathrm{MA}$

5.00

MA

5.00

MA

5.00

Semple Date 


\section{C -30}

KANSAS CITY : SELECTEO AMALYSIS FROM KC91.158-L

Sumple Date

Constituent

$\omega / L$

$10 / 07 / 91$

\begin{tabular}{|c|c|}
\hline FLLOROTRICHLOROME TKAME & 10.00 \\
\hline HEXACHLOROQUTADIEME & MA \\
\hline I SOPROPYLBENZENE & MA \\
\hline M-XYLENE & MA \\
\hline METHYL BRONIDE & 10.00 \\
\hline METHYL CHLORIDE & 10.0 \\
\hline METHYLENE CHLORIDE & 5.00 \\
\hline M-BUTYLBENZENE & MA \\
\hline N-PROPYLBENZENE & NA \\
\hline NAPHTKALENE & MA \\
\hline O-XYYLEKE & NA \\
\hline P-I SOPROPYLTOLUENE & HA \\
\hline P•XYLEKE & MA \\
\hline SEC-EUTYLBENZENE & MA \\
\hline STYRENE & 5.00 \\
\hline TERT-WUTYLBENZENE & MA \\
\hline TETRACKLOROETHENE & 5.00 \\
\hline TOLUENE & 5.0 \\
\hline TPH & MA \\
\hline TPHA & WA \\
\hline TPKL & NA \\
\hline TRAMS-1,2-DICKLOROETHENE & NA \\
\hline TRAKS-1,3-DICHLOROPROPENE & 5.00 \\
\hline TRICHLOROETKEHE & 5.ov \\
\hline VINYL ACETATE & NA \\
\hline XYLENES (total) & $5 . \infty$ \\
\hline
\end{tabular}


KANSAS CITY : SELECTED AMALYSIS FROM KC91.158-U

Sample Date

constitient

$\omega / L$

10/07/91

$1,1,1,2$ - TETRACHLOROETHANE MA

$1,1,1$-TRICHLOROETHANE 5.0

$1,1,2,2$-TETRACHLOROETHAME 5.00

$1,1,2$-TRICHLOROETHANE S.W

1,1,2-TRICHLOROTRIFLLOROETH S.W

1,1 -OICHLOROETHANE S.0U

1,1 -DICHLOROETHENE 5.00

1,1 -DICHLOROPROPENE KA

$1,2,3$-TRICKLOROBENZENE NA

1,2,3-TRICHLOROPROFANE KA

1,2,3-TRIKETHYLEEREENE HA

$1,2,4$-TRICHLOROBEKZENE HA

1,2-DIBROMO-3-CHLOROPROPANE MA

1,2 -DIBROMOETHANE NA

1,2 -DICKLOROBEMZENE 5.00

1,2-01CHLOROE THANE 5.as

1,2 -DICHLOROETHENE (10T:1) 5.00

1,2-DICHLOROPROPANE 5.0U

1,3,5-TRIMETHYLBENZENE MA

3-DICHLOROBEMZENF 5.00

1,3-DICKLOROPROPAKE MA

1,4-DICHLOROBENZENE 5.00

2,2-DICHLOROPROPAME HA

2-BUTANONE $5.0 \mathrm{~S}$

2-CHLOROETHYLVIHYL ETHER MA

2-CHLOROTOLUEME MA

2- HEXanONE S.OU

4-CMLOROTOLUENE HA

4-METHYL-2-PEHTANOWE 5.00

ACETONE 10.0

ACROLEIK

ACRYLOWITRILE MA

BENZENE 5.00

BROHOBENZENE

BROHOCHLOROMETHANE MA

OROMOFORM 5.01

CARRON DISULFIDE $5.0 \mathrm{~W}$

CARBOW TETRACHLORIDE 5.0

CHLOROQENZEME S.OS

CHLORCO IBROMOMETHANE 5.00

CHLOROETHANE $\quad 10.00$

CHLOROETHEME 10.00

CHLOROFORM 5.00

CI5-1,2-0ICKLOROETHENE MA

-15-1,3-01CKLOROPROPENE 5.00

IBronomethake Ha

DICHLORCBROMOMETKANE $5.0 \mathrm{~d}$

OICHLORCOIFLLOROMETHANE MA

ETHYLBENZEME S.OU 
RANSAS CITY : SELECTED AMALYSIS FROM KC91-158-U

Consti tuent

$10 / 07 / 91$

FLVOROTRICHLOROMETHANE

10.00

HEXACHLOROQUTADIENE

MA

ISOPROPYLBENZENE

K-XYLENE

NA

METHYL BROMIDE

METHYL CHLORIDE

MA

10.00

METHYLENE CHLORIDE

10.00

N-BUTYLBENZENE

$5 . \infty$

MA

N-PROPYLBENZENE

MAPHTHALENE

O-XYLENE

P-ISOPROPYLTOLUENE

P-XYLENE

SEC-BUTYLBENZENE

STYRENE

TERT-BUTYLBENZENE

TETRACHLOROETHENE

NA

NA

NA

NA

NA

WA

5.00

NA

5.00

5.0

TOLUENE

TPH

NA

TPHK

IPHL

HA

MA

TRANS-1,2-DICHLOROETHENE

TRANS-1,3-DICKLOROPROPENE

TRICHLOROETHENE

VINYL ACETATE

5.00

5.00

NA

XYLENES (total)

Sample Date 
KANSAS CITY : SELECTED AHALYSIS FROM KC91-159-L

Constituent

ug/L

$1,1,1,2$ - TETRACHLOROETHANE

$1,9,1$ - TRiCHLOROETHAME

$1,1,2,2-$ TETRACHLOROETHANE

$1,1,2$-TRICHLOROETKANE

1,1,2-TRICHLOROTRIFLLOROETH

1,9 -DICHLOROETHAME

1,1-DICHLOROETHENE

1,1-DICHLOROPROPEME

$1,2,3$ - TRICHLOROBENZENE

$1,2,3$-TRICHLOROPROPANE

$1,2,3$-TRIMETHYLBENZENE

$1,2,4$-TRICKLOROBENZENE

1,2-D1BRONO-3-CHLOROPROPANE

1,2 -DIBROMOETHANE

1,2 -DICHLOROBENZENE

1,2-DICHL OROETHAHE

1,2-DICHLOROETHENE (total)

1,2 -OICHLOROPROPANE

$1,3,5$ - TRIMETHYLBENZENE

,3-DICHLOROBEN2ENE

1,3-DICHLOROPROPANE

1,4-DICHLOROBEN2ENE

2,2-DICHLOROPROPANE

2-BUTAKONE

2-CHLOROETHYLVIHYL ETHER

2-CHLOROTOLUENE

2- MEXAMONE

6-CHLOROTOLUENE

6- METHYL-2-PENTANONE

ACE TONE

ACROLEIN

ACRYLOWITRILE

BENZENE

BROMOBENZENE

BRONOCHL OROME THANE

BRONOFORM

CARBON DISULFIDE

SARBOW TETRACKLORIDE

CMLOROBENZENE

CHLORCOIBROMOMETHANE

CHLOROETKANE

CHLOROETHEME

CHLOROFORM

C15-1,2-01CHL DROE THENE

C.15-1,3-01CHLOROPROPEME

IBROMOME TMANE

DICHLOROBROMOMETHANE

DICHLORDO I FLLOROME TMAKE

ETMYLBENZENE
NA

8.0

5.0

5.00

5.00

22.0

9.0

NA

NA

NA

NA

NA

NA

NA

5.00

5.00

250.0

5.00

NA

s.ou

NA

5.0

MA

5.00

NA

NA

5.00

na

5.00

10.0

WA

NA

5.00

HA

MA

5.0

5.00

5.00

5.00

5.00

10.00

10.00

5,00

Ma

5.00

NA

5.00

NA

5. os

Sample Date 


\section{C -34}

KANSAS CITY : SELECTEO ANALYSIS BROA KCO1-159-L

Sample Date

constituent

10/07/91.

\begin{tabular}{|c|c|}
\hline FLUOROTRICHLORONETHANE & 10.0 \\
\hline REXACHLOROQUTADIENE & MA \\
\hline ISOPROPYLBENZENE & HA \\
\hline M-XYLENE & MA \\
\hline METHYL BROAIDE & 10.00 \\
\hline METHYL CHLORIDE & 10.00 \\
\hline METHYLENE CHLORIDE & $5 . \infty$ \\
\hline N-BUTYLBENZENE & MA \\
\hline N-PROPYLBENZENE & ma \\
\hline NAPKTHALENE & MA \\
\hline $0 \cdot X Y L E N E$ & NA \\
\hline P-I SOPROPYLTOLUENE & NA \\
\hline P·XYLENE & NA \\
\hline SEC-BUTYLEENZENE & Ma \\
\hline STYRENE & 5.00 \\
\hline TERT-BUTYLBENZENE & MA \\
\hline TETRACHLOROETHENE & 5.00 \\
\hline TOLUENE & $5.0 \mathrm{~s}$ \\
\hline TPH & WA \\
\hline TPHK & MA \\
\hline TPKL & MA \\
\hline TRANS-1,2-DICHLOROETHEME & MA \\
\hline TRAHS-1,3-DICHLOROPROPENE & 5.00 \\
\hline TRICHLOROETHENE & 21.0 \\
\hline VINYL ACETATE & MA \\
\hline XYLENES (rotal) & 5.00 \\
\hline
\end{tabular}


RAKSAS CITY : SELECTED AMALYSIS FROA XC99-159-U

ionstituent
$u g / L$

$1,1,1,2$ - TETRACHLOROETHRNE

$1,9,1$-PRICHLOROETHANE

$1,1,2,2$ - TETRACHLOROETHAME

$1,1,2$ - TRICKLOROETHANE

$1,1,2$-TRICHLOROTRIFLUOROETH

1, 1-OICHLOROETHAKE

1,1-DICHLOROE THEME

1,1-DICHLOROPROPENE

$1,2,3$ - TRICHLOROBENZENE

1,2,3-TRICHLOROPROPANE

$1,2,3$-TRIMETHYLBENZENE

$1,2,4$-TRICHLOROBENZENE

1,2-01BROMO-3- CHLOROPROPANE

1,2-DIBromoEt HAME

1,2-HICHLOROBEKZENE

1,2-DICHLOROETHAHE

1,2-DICHLOROETHENE (total)

1,2-DICHLOROPROPANE

1,3,5-THIGETHYL BENZENE

3-DICHL.OROBENZENE

,3-DICHLDROPROPANE

1,4-DICHLOROBEHZEME

2,2-DICHLOKOPROPANE

2- BUTANOME

2-CHLOROETHYIVIKYL ETHER

2-CHLOROTOLUENE

2- KEXANOWE

4-ChLOROTOLUENe

4-METHYL-2-PENTANONE

ACETONE

ACROLEIN

ACRYLOWITRILE

BENZENE

BROMOBENZENE

BROHOCHL ORCME THANE

BROAFFORK

CARBOW DISULFIDE

CARBOW TETRACHLORIOE

CHLOROBENZENE

CHLOROO I BROMONE THAKE

CHLOROETHANE

CHLOROETHENE

CHLOROFORM

CIS-1,2-DICHLOROET THE ME

TIS-1,3-DICHLOROPROPENE

IBROAOME THAHE

DI CHL OROBROMOKE THANE

DICHLORCO I FLUOROMETHAHE

ETHYLBEHZENE
NA

5.01

5.0u

5.00

5.01

21.0

21.0

MA

MA

NA

MA

MA

NA

MA

5.0

5.00

210.0

5.00

MA

5.0u

wa

5.00

MA

5.00

MA

MA

5.00

$\mathrm{MA}$

5.0s

10.00

MA

MA

5.0u

MA

MA

5.01

5.00

s.os

5.00

5.00

10.00

10.00

5.00

MA

$5.0 \mathrm{~V}$

MA

s.o

MA

5.0
Sample Date 


$$
\text { C }-36
$$

KAKSAS CITY : SELECTED AMALYSIS FROA KCO1-159-U

Semple Date

Constivient

$\omega / l$

$10 / 07 / 91$

\begin{tabular}{|c|c|}
\hline FLUOROTRICHLORONETHANE & $10 . w$ \\
\hline MEXUCHLOROQUTAOIENE & $\mu$ \\
\hline ISOPROPYLBEWZENE & MA \\
\hline M-XYLENE & M \\
\hline METHYL BROMIDE & 10.0 \\
\hline METHYL CHLORIDE & 90.0 \\
\hline METKYLENE CHLORIDE & s.w \\
\hline M-BUTYLBENZENE & WA \\
\hline N-PROPYLBENZEME & HA \\
\hline MAPHTHALENE & MA \\
\hline O-XYLENE & NA \\
\hline P-ISOPROPYLTOLUENE & NA \\
\hline$P \cdot X Y L E N E$ & NA \\
\hline SEC-QUTYLBENZENE & NA \\
\hline STYRENE & 5.0 \\
\hline TERT-BUTYLBENZENE & MA \\
\hline TETRACHLOROETHENE & 5.00 \\
\hline TOLUENE & 5.0 \\
\hline TPH & MA \\
\hline ТРНн & MA \\
\hline TPML & MA \\
\hline TRAMS-1,2-DICHLOROETHENE & NA \\
\hline TRANS-1,3-01CMLOROPROPENE & $5 . \infty$ \\
\hline TRICHLOROETHENE & 16.0 \\
\hline VINYL ACETATE & MA \\
\hline XYLENES (total) & 5.00 \\
\hline
\end{tabular}




$$
C-37
$$

KALSAS CITY : SELECTED AKALYSIS FROA KES9-159-L

Constitueris

us/L

$9,1,1-$ iR/CHLOEDETHRHE

$1,1,2,2 \cdot$ IEIRACHLLOROETHAHE

$1,1,2$ - IRICHLOLOETHALE

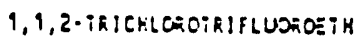

1,9.0ICHLCEOETHALE

$1,9 \cdot 0$ ICHLORE IHELE

$1,2 \cdot$ DICKL OROSERTELLE

1,2-DICHLOAOETH.AKE

1,2-D!CKLCAOSTHERE (iCTEl)

1,2-DICHLCADRKCTALE

1,3-01CHLOROEEKZENE

1,6 -01CMLOACOERZEKE

2. BUTAKDIE

2- HEXAHONE

4- METHYL-2-FELTAKONE

ACETONE

BEHZENE

BROHOFOEM

CAREON CISULFIDE

CAREON TETKACKLORIEE

CHLOROSERLZLLE

CHLOROSI BROMOMETHALE

CKLOROET TRLEE

CKIOROETHERE

CHLOEOFOQM

CIS-9,3-CICHLOFOOROOEKE

DICHLORESROMDAETHALE

ETHYLEENZENE

FLUSROTK/CKLOROAETHALE

METHYL BROMIDE

METHYL CHLOZIOE

RETHYLLEE CHLORIDE

STYRELE

TETRACMLOKOE THEME

TOLUENE

IPFH

TFHL

TRALS-9,3-0ICHLOROPKOTERE

TRICHLOROETHENE

XYLERES (TOIAL)
Somfle date

$64: i 5 / 52$

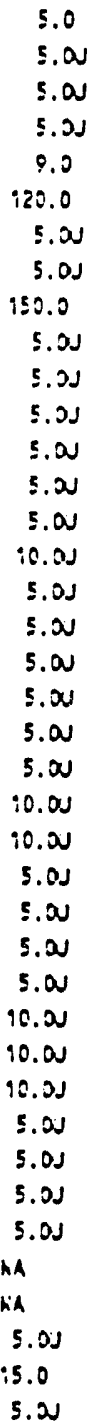


KAKSAS CITY : SELECTED ANALYSIS IROM KC99-159-U

Constituent

ug/L

\begin{tabular}{|c|c|}
\hline $1,9,9$-TRICHLOROETKANE & 5.0 \\
\hline 1,1,2,2- IETRACHLOROETHANE & 5.01 \\
\hline 1,1,2-TRICHLOROETKAKE & s.as \\
\hline 1,1,2-TRICHLOROTRIFLUOROETH & 5.a \\
\hline 1,9-OICHLOROETHAHE & 19.0 \\
\hline 1.1.DICHLOROET HEME & 17.0 \\
\hline 1,2-DICHLORO5EN2EHE & 5.00 \\
\hline 1,2-DICKLOROEThAHE & 5.0 \\
\hline 1,2-0ICYLOROETHENE (TOTS1) & 1800.0 \\
\hline 1,2-DICHLOROFRORAHE & 5.00 \\
\hline 1,3-DICKLOROSERIZENE & 5.00 \\
\hline 1,4-DICKLOROSENRZENE & 5.00 \\
\hline 2-BUTAMOME & s.ou \\
\hline 2- HEXANOWE & 5.00 \\
\hline 6-METMYL-2-PEKTANOHE & 5.00 \\
\hline ACETONE & $10.0 \mathrm{~s}$ \\
\hline BENZEME & 5.00 \\
\hline BRONOFORM & 5.0S \\
\hline CARBOW DISULFIDE & 5.00 \\
\hline CARBON TETRACHLORIDE & 5.00 \\
\hline CHLOROBENZEHE & 5.00 \\
\hline CHLOROOI BROHOMET HANE & 5.00 \\
\hline CHLOROETHANE & 10.0 \\
\hline CHLOROET THENE & 10.0 \\
\hline CKLOROFORM & 5.00 \\
\hline CIS-1,3-DICHLOROPROPENE & 5.00 \\
\hline DICKL OROBROMOMET TKANE & 5.00 \\
\hline ETHYLBENZENE & $5.0 \mathrm{~s}$ \\
\hline FLLOROTRICHLOROME TKANE & 10.00 \\
\hline METHYL BRONIDE & 10.00 \\
\hline METHYL CHLORIDE & $10.0 \mathrm{~s}$ \\
\hline METHYLENE CHLORIDE & 5.00 \\
\hline STYREHE & 5.00 \\
\hline TETRACHLOROETHENE & 5.0u \\
\hline TOLUENE & $5.0 \mathrm{~s}$ \\
\hline TPHH & MA \\
\hline TPHL & MA \\
\hline TRAMS-1,3-DICKL OROPROPENE & 5.0 \\
\hline TRICHLOROE THENE & 30.0 \\
\hline XYIENES (totهl) & $5 . \alpha$ \\
\hline
\end{tabular}

$04 / 15 / 92$

as

.os

.00

5.0

. 00

.$\infty$

.0

s.os

. $\infty$

5.00

0.00

.$\infty$

.ou

.ou

5.00

5.00
Somple Date 
KANSAS CITY : SELECTED AKALYSIS FROM KC91-161-L

Constituent

ug/L

$1, ?, 1 \cdot$ TRICHLOROETHANE

$1,1,2,2$-TETKACHLOROE THANE

$1,1,2$-TRICHLOROETHANE

1,1,2-TRICHLOROTR! FLUOROETH

1,9-DICHLOROETHANE

1,9-DICHLOROETHENE

1,2-DISHLOROGENZENE

1,2-DICHLOROET TAME

1,2-DICHLOROETHENE (TOTOl)

1,2-DICHLOROPROFAKE

1,3-DICHLOROBENZENE

1,6-DICHLOROSEKZENE

2-BUTAROME

2- HEXANOWE

4-METKYL-2-PEHTAHONE

ACETONE

BENZEHE

BROMUFORM

CARBOW DISULFIDE

CARBOW TETRACKLORIDE

CHLOROSELZENE

CHLORDOI GROMOMETHANE

CHLOROETHAKE

CHLOROETHENE

CHLOROFORM

CIS-1,3-DICHLOROPROPENE

DICHLOROSROMOMETHANE

ETHYLBENZENE

FLUOROTRICHLOROMETHAME

METHYL BROMIDE

METHYL CHLORIDE

METHYLEKE CHLORIDE

STYRENE

TETRACHLOROETHEME

TOLUENE

TPHH

TPHL

TRANS-1,3-DICHLOROPROPENE

TRICHLOROETHENE

XYLENES (rOtol)
Sumple Dote
$06 / 16 / 92$
5.00
5.00
5.00
5.00
10.0
5.00
5.00
5.00
200.0
5.00
5.00
5.00
5.00
5.00
5.00
10.00
5.00
5.00
5.00
5.00
5.00
5.00
$10.0 \mathrm{~s}$
10.00
5.00
5.00
5.00
5.00
10.00
10.00
10.00
5.00
5.00
5.00
5.00
na
NA
5.00
6.0
5.00 
Constituent

$u s / L$

$1,1,1$ - TRICHLOROE THANE

$1,1,2,2$-TETRACHLOROETHANE

$1,1,2$-TRICHLOROETHANE

$1,1,2$-TRICHLOROTRIFLLOROETH

1,1 -DICHLOROETHANE

1,1 -DICHLOROETHENE

1,2-D1CHLOROBENZENE

1,2-DICHLOROETHAHE

1,2-DICHLOROETHENE (total)

1,2-DICHI OROPROPANE

1,3-DICKLOROBENZENE

1,4-DICKLOROBENZENE

2-BUTANONE

2- HEXANONE

4-METHYL-2-PENTANONE

ACE TONE

BENZENE

BROWOFORM

CARBOW DISULFIDE

CARBCA TETRACHLORIDE

CHLOROBERZENE

CHLOROO I6ROMOMETHANE

CHLOROETHANE

CHLOROETHENE

CKLOROFORK

CIS-1,3-DICHLOROPROPENE

DICHLOROBROMOAET HAME

ETHYLBENZENE

FLUDROTRICHLOROMETHANE

METHYL BROMIDE

METKTL CHLORIOE

METHYLENE CHLORIDE

STYRENE

TETRACHLOROETHENE

TOLUEHE

ТРHK

TPKL.

TRANS-1,3-DICHLOROPROPEHE

TRICHLOROETHEHE

XYLENES (totol)
$04 / 94 / 52$

5.00

5.00

s. 0

5.00

5.00

5.0

5.0

5.00

210.0

5.00

5.00

5. $\infty$

5. O

5.00

5.00

10.01

5.00

5. 0

5.00

5. 0

5. 0

5.00

$10.0 \mathrm{~s}$

10.00

5.00

5.00

5.0

5.00

10.0

10.0

$10.0 \mathrm{~s}$

5.00

5.0

5. 0

s.0

ina

NA

5.00

5.00

5.00 


\section{C -41}

KANSAS CITY : SELECTED ANALYSIS FROM XC91-163.L

Constituent

ug/L

$1,1,1,2$-TETRACHLOROETHANE

$1,1,1$ - TRICHLOROETHANE

$1,1,2,2$-TETRACHLOROETHAME

$1,1,2$ - TRICHL OROETHANE

$1,1,2$ - TRICHLOROTRIFLUOROETH

1,1-DICHLOROETHANE

1,1-DICHLOROETHENE

1,1-DICHLOROPROPENE

$1,2,3-$ TRICHLOROBENZENE

$1,2,3$-TRICHLOROPROPANE

$1,2,3$-TRIMETHYLBENZENE

$1,2,4$-TRICHLOROBEHZENE

1,2 -0IBROMO-3-CHLOROPROPANE

1,2 -DIBROMOETHANE

1,2 -DICHLOROBENZENE

1,2-DICHLOROETHANE

:. 2-DICHLOROETHEHE (total)

1,2-D: CHLOROPROPANE

1,3,5-TRIMETKKLLENZENE

, 3-01CHLOROBENZENE

1,3-O1CHLOROPROPANE

1,4-DiCHLOROBENZENE

2,2-DICHLOROPROPANE

2- BUTAMONE

2-CHLOROETHYLVINYL ETHER

2-CHLOROTOLUENE

2- HEXANOWE

4-CHLOROTOLUENE

4-METHYL-2-PENTANOME

ACETOME

ACROLEIN

ACRYLOWITRILE

BENZENE

BROMOBENZENE

BRONOCHL ORCME THANE

BROMOF ORM

CARBON DISULFIDE

CARBOW TETRACHLORIDE

CHLOROSENZENE

CHLOROOI I ROMOMETHANE

CHLOROET TAME

CHLOROE THENE

CHLOROFORH

CIS-1,2-OICHLOROETHENE

SIS-1,3-0ICHLOROPROPENE

IBROMOME THANE

DICHLOROBROMOME THANE

DICKLOROOIFLLOROME THAME

ETHYLBENZEME
NA

32.0

5.00

s. 01

5.00

190.0

91.0

MA

NA

MA

MA

MA

MA

KA

5.00

12.0

290.0

5.00

NA

5.00

MA

5.00

MA

5. 01

MA

MA

5.00

$\mathrm{ma}$

5.00

10.00

MA

wa

s.or

ma

MA

5.00

5.00

s.ou

5.00

5.00

10.00

48.0

5.0

MA

s.0s

Ma

5.00

MA

s.a

Sample Date 
RANSAS CITY : SELECTED AKALYSIS FROA KC91-163-L

Constituent

us/l

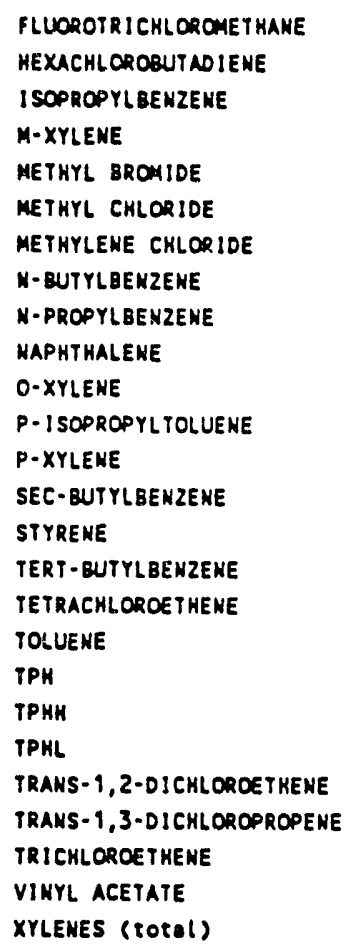

Sample Dote
10.0

NA

MA

MA

10.00

10.00

5.0

MA

KA

NA

Na

NA

MA

NA

5.00

NA

5.0

5.0

MA

MA

NA

NA

5.00

37.0

nA

5.0u 


\section{C -43}

KANSAS CITY : SELECTED AMALYSIS FROM KC91-163-U

Constituent

ug/t

$1,1,1,2$ - TETRACHLOROE THANE

$1,1,1$-TRICHLOROETHANE

$1,1,2,2$ - TETRACHLOROE THANE

$1,1,2$-TRICHLOROETHANE

$1,1,2$-TRICHLOROTRIFLLOROETH

1,1 -DICHLOROETHANE

1,1-DICKLOROETHENE

1,1-OICHLOROPRODENE

$1,2,3$-TRICHLOROBENZENE

1,2,3-TRICHLOROPROPANE

$1,2,3$ - TRIME THYLBENZENE

$1,2,6$-TRICHLOROBENZENE

1,2-01BROMO-3-CHLOROPROPANE

1,2-DisRomoEt HAKE

1,2-DICHLOROBENZENE

1,2-DICHLOROETHAME

1,2-DICHLOROETHENE (TOTal)

1,2-DICHLOROPROPANE

$1,3,5-$ TRJMETHYLBENZENE

3-DiChLOROBENZENE

1,3 -DICHLOROPROPANE

1,6 -DICHLOROBENZENE

2,2-0ICHLOROPROPANE

2- BUTANONE

2-CHLOROETHYLVINYL ETHER

2-CHLOROTOLUEME

2- HEXAMONE

4-CHLOROTOLUENE

4-METHYL-2-PENTAKONE

ACETONE

ACROLEIK

ACRYLONITRILE

BENZEME

BROMOBENZENE

BROMOCHL ORCMET THAME

EROMOFORM

CARBOA DISULFIDE

CARBOA TETRACHLORIDE

CHLOROBEHZENE

CHLOROOI IBROMOMETHANE

CHLOROET HANE

CHLOROE TKENE

CHLOROFORM

C15-1,2-OICHLOROE THENE

-IS-1,3-DICHLOROPROPENE

IBROMOMETHAME

DICHLOROBROMOMET THAME

DICHLORDOIFLLOROME THANE

ETHYLBENZENE
MA

5.00

s.ou

5.00

5.00

5.04

5.01

MA

MA

WA

ma

Ma

ma

na

5.00

8.0

290.0

5.00

na

5.00

HA

5.0

HA

5.00

MA

MA

5.0u

kA

5.00

10.00

NA

WA

5.00

MA

MA

5.00

5.00

5.00

5.00

5.00

10.01

90.0

5.00

HA

5.00

MA

5.00

NA

5.00

Somple Date 
KANSAS CITY : SELECTED AMALYSIS FROM KC91.163.U

Constituent

ugh

FLUOROTRICHLOROMETHAKE

HEXACHLOROBUTNDIENE

ISOPROPYLBENZENE

M-XYLENE

METHYL BROMIDE

METHYL CHLORIDE

METHYLENE CHLORIDE

N-BUTYLBEKZENE

N-PROPYLBENZENE

MAPKTHALENE

O-XYLENE

P-I SOPROPYLTOLUENE

P.XYYEME

SEC-BUTYLBENZENE

STYREME

TERT-BUTYLBENZENE

TETRACHLOROETHENE

TOLUEME

IPH

TPHH

TPHL

TRANS-1,2-DICHLOROETHENE

TRANS-1,3-01CKLOROPROPENE

TRICHLOROETHEME

VINYL ACETATE

XYLENES (TOTAl)
Sample Dase

$10 / 08 / 91$

10.00
$M A$
$M A$
$M A$
10.00
10.00
5.00
$M A$
$M A$
$M A$
$M A$
$M A$
$M A$
$M A$
5.00
$M A$
5.00
5.00
$M A$
$M A$
$M A$
$M A$
5.00
11.0
$M A$
5.00

10.00

10.00

5.00

5.00

A

5.00

MA

MA

5.00

11.0

5.00 
RANSAS CITY : SELECTED AKALYSIS IROA KCQ9-163-L

Sumple Date

Constituent

$u / h$

$04 / 94 / 92$

\begin{tabular}{|c|c|}
\hline 1,1,1-TRICKLOROETHAHE & 23.0 \\
\hline $1,1,2,2$-TETKACHLOROETHANE & 5.00 \\
\hline $9,1,2-T R I C H L O 2 O E T$ KANE & 5.00 \\
\hline 1,1,2-TRICHLOROTRIFLUOROETH & 5.00 \\
\hline 1,1-DICKLOROETHAKE & 120.0 \\
\hline 1,1-DICHLOROE THEKE & 100.0 \\
\hline 1,2-DICHLOROSELIZENE & $5.0 \mathrm{~J}$ \\
\hline 1,2-DICHLOROETHANE & 5.04 \\
\hline 1.2-DICHLOROETHENE (TOTAl) & 9600.0 \\
\hline 1,2.DICHLOROPROFANE & 5.00 \\
\hline 1,3-DICHLOROSEKZEKE & 5.00 \\
\hline 1,4-DICHLOROSENZENE & 5.00 \\
\hline 2-BUTANONE & 5.00 \\
\hline 2- MEXANOWE & 5.00 \\
\hline 4-METHYL-2-PEHTANONE & 5.00 \\
\hline ACETOWE & $10.0 \mathrm{~J}$ \\
\hline BENZEME & 5.00 \\
\hline BROMOFORK & $5.0 \mathrm{~J}$ \\
\hline CARBOW DISULFIDE & s.0u \\
\hline CARBOW TETRACHLORIDE & 5.00 \\
\hline CHLOROBENZENE & 5.04 \\
\hline CHLORDO I BROM SMETHANE & 5.00 \\
\hline CHLOROETHANE & 10.00 \\
\hline CHLOROETHENE & 36.0 \\
\hline CHLOROF ORM & 5.00 \\
\hline CIS-1,3-DICKLOROFROPENE & 5.00 \\
\hline DICHLOROSROMLMET THANE & 5.00 \\
\hline ETHYLBEKIEENE & 5.00 \\
\hline FLLOROTRI CKL OROMETHANE & 10.00 \\
\hline METHYL BROMIDE & 10.00 \\
\hline METHYL CKLORIDE & $10 . \alpha$ \\
\hline METHYLENE CHLORIDE & 5.00 \\
\hline STYRENE & $5 . \infty$ \\
\hline TETRACKLOROETHEHE & 5.00 \\
\hline TOLUEWE & 5.00 \\
\hline TPHH & $\mathrm{ma}$ \\
\hline TPHL & wa \\
\hline TRANS-1,3-DICHLOROPROPENE & 5.00 \\
\hline TRICHLOKOETHEME & 26.0 \\
\hline XYLENES (roinl) & 5.00 \\
\hline
\end{tabular}


KANSAS CITY : SELECTED ANALYSIS FROM KCQ9.1E3.U

Constituent

Smple Dote

ug/

$1,1,9 \cdot$ TRICHLOROETKANE

$1,1,2,2$-TETRACHLOROETHAHE

$1,1,2$ - TRICHLOROETHAKE

$1,1,2$-TRICHLOROTRIFLUOROETH

1,9-Dichloroet thane

1,1-D1 SMLOROETHELE

1,2-DICHLOROBEHZELE

1,2-DICHLOROE T MAHE

1,2-DICHLOROETMEME (TOTal)

1,2-01 CMLOROPROPANE

1,3-DICHLOROEEMZENE

1,6-D!CHLOROSENZENE

2-BUTAMONE

2- HEXAMOME

4-METHYL-2-PEHTAMOHE

ACETOWE

DENZENE

BRONOFORM

CARBOW DISULFIDE

CARBON TETRACHLORIDE

CHLOROBEWIZEME

CHLORCOIBROMOMETHAKE

CHLOROETHANE

CHLOROETHENE

CHLOROFORM

CIS-9,3-DICHLOROPROPEME

OICHLOROBROMOME TMAHE

ETHYLBENZENE

PLUOROTRICHLOROMETHANE

KETHYL DROMIDE

METHYL CHLORIOE

METHYLENE CHLORIDE

STYREME

TETRACHLOROE THEME

TOLUEHE

IPMH

IPKL

TRANS-1,3-DICHL OROPROPENE

TRICHLOROE THEME

XYLEMES (rOtal)
$06 / 96 / 92$

5.00

5.00

5.00

5.00

5.00

5.00

5.00

5,00

220.0

5.00

5.00

5.00

5.00

5.00

5.00

10.00

5.0u

5.00

5.00

5.00

5.00

5.00

10.00

110.0

5.04

5.00

5.00

5.00

10.0

10.00

10.04

5.00

5.01

5.00

5.00

NA

HA

5.01

12.0

5.00 
KANSAS CITY : SELECTED AKALYSIS FROM KC99-164-L

Constituent

$u g / L$

$10 / 19 / 91$
1,1,1,2-TETRACHLORDETHANE

$1,1,9$ - TRIEHLOROETHANE

$1,1,2,2$ - TE TRACKLOROE TKANE

$1,1,2$-TRICHLOROETHANE

1,1,2-TRICHLOROTRIFLLOROETH

1,1-DICHLOROETHANE

1,1-DICHLOROE THENE

1,1 -DICHLOROPROPENE

$1,2,3$ - TRICHLOROBENZENE

1,2,3-TRICHLOROPROPANE

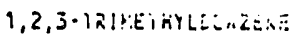

$1,2,4$-TRICKLOROBENZENE

1,2-018ROMO-3-CHLOROPROPANE

1,2-DIBROMOETHANE

1,2-DICHLOROBENZENE

1.2-DICHLOROET TANE

1,2-DICHLOROETHENE (total)

1,2-DICKLOROPROPANE

1,3,5-TRIMETHYLBENZENE

'3-DiCHLOROBENZENE

i, 3-OICHLOROPROEVARE

1,4-DICHLOROBENZEME

2, 2-DICHLOROPROPAHE

2-BUTANONE

2- CHLOROETHYLVIMYL ETHER

2-CHLOROTOLUENE

2- HEXAMONE

4-CHLOROTOLUENE

4-METHYL-2-PENTANONE

ACE TONE

ACROLEIK

ACRYLOWITRILE

Denzene

BROMOBENZENE

BROMOCHL ORONE THAKE

BROWOF ORM

CARBOW DISULFIDE

CAREON TETRACHLORIDE

CHLOROBENZENE

CHLORDO IBROMOME THANE

CHLOROETHANE

CHLOROETHENE

CHLOROFORM

CIS-1,2-DICHLOROETHENE

T.1S-1,3-D1CHLOROPROPENE

I6rOMOMETHANE

DICHLOROBRomone T HANE

DICHLORCOIFLLOROMET THANE

ETKTLBENZEHE
NA

5.00

5.01

5.00

5.00

5.00

5.00

MA

MA

ma

...

MA

NA

MA

5.0U

5.00

5.0

5.00

MA

5.00

MA

5.00

IIA

5.04

MA

Ma

5.00

MA

5.00

10.w

$M$

MA

5.0u

MA

NA

S.00

5.00

5.00

5.00

5.00

10.01

10.01

5.00

HA

5.00

MA

s.ov

MA

5.0
Semple Dete 


\section{C -48}

KANSAS CITY : SELECTED AMALYSIS FROM XC91-164-L

Sample Date

Constituent

ug $/ \mathrm{L}$

$10 / 11 / 91$

FLLOROTRICHLORONETKANE

10.0

MEXACHLOROQUTADIENE

MA

ISOPROPYLBEKZENE

M-XYLEME

METHYL BROMIDE

METHYL CHLORJOE

METHYLENE CKLORIDE

H.BUTYLBENZENE

MA

MA

10.00

10.00

5.00

N-PROPYLBENZENE

MAPHTHALENE

MA

MA

MA

O.2..EE

iis

P- I SOFROPYLTOLUERE

P-XYLEME

NA

MA

SEC-BUTYLBENZENE

STYRENE

MA

5.00

TERT-QUTYYLENZENE

MA

s.0u

TETRACKL OROE THENE

5.00

TPH

MA

TPHK

MA

TPHL

MA

TRANS- $9,2-01$ CKLOROET THENE

NA

TRAKS-1,3-DICHLOROFROPEKE 5.00

TRICHLOROETHENE S.0U

VINYL ACETATE

Ma

XYLENES (total)

5.00 
KANSAS CITY : SELECTED AMALYSIS FROM XC99-166-U

ions tituent

$u g / h$

1,1,1,2-tetrachloroethaNe

$1,1,1$-TRICHLOROETHANE

$1,1,2,2$-TETRACHLOROETHANE

$1,1,2$ - TRICHLOROE THANE

$1,1,2$ - IRICHLOROTRIFLUOROETH

1,1-DICHLOROETHANE

1,1-DICHLOROET TENE

1,1 -DICHLOROPROPENE

$1,2,3$-TRICKLOROSENZEME

$1,2,3$ - TRICHLOROPROPANE

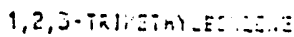

$1,2,6$-TRICHLOROBENZENE

1,2 -D1BROMO-3-CHLOROPROPANE

1,2-DIBROMOE THAHE

1,2 -DICHLOROBENZENE

1,2-DICHLOROETHAME

1,2-01CHLORCETHEHE (total)

1,2-DICHLOROPROPANE

1,3,5-TRIMETHYLBENZENE

3-DICHLOROBEN2ENE

, 3-DICHLOROPROPANE

1,4-DICHLOROSENZENE

2,2-DICHLOROPROPANE

2- BUTANONE

2-CHLOROETHYLVIKYL ETHER

2-ChLOROTOLUENE

2- KEXANONE

4-CHLOROTOLUENE

4-HETHYL-2-PENTANOKE

ACETONE

ACROLEIN

ACRYLOWITRILE

BENLENE

BROMOBENZENE

BROMOCHLOROMET HAME

BROMOFORM

CARBOW DISULFIDE

CARBON TETRACHLORIDE

CHLOROBENZENE

CHLORCOI IBROMOMET TAME

CHLOROE THANE

CHLOROETHEME

CHLOROFORM

C15-1,2-DICHLOROETHENE

C.15-1,3-0ICHLOROPROPENE

:BROMOMETHANE

JICHLOROBROMOME T TANE

DICHLORCOIFLLOROMET HANE

ETHYLBENZENE
HA

5.01

5.00

5.00

5.00

5.00

5.00

NA

MA

NA

Ki

NA

WA

NA

5.00

5.00

5.00

5.00

NA

5.00

WA

5.00

HA

5.00

MA

HA

5.00

MA

?.\%)

10.w

$\mathrm{MA}$

MA

5.0

MA

MA

5.00

5.00

5.00

5.00

5.00

10.00

10.01

5.00

*a

5.01

ma

5.00

MA

s.0u

Smple Date

Taple Date 


\section{C -50}

RANSAS CITY

: SELECTED AKALYSIS FROM XC91-164-U

Sumple Date

Constituent

us/l

FLLOROTRICHLOROMETHAHE

HEXUCHLOROBUTADIENE

ISOPROPYLBENZENE

M-XYLENE

METHYL BROMIDE

METHYL CHLORIDE

METKYLENE CMLORIOE

N-BUTYLBEXZENE

N-PROPYLBENZENE

MAPHT KALENE

C...

P-1 SOPROPYLTOLUENE

P-XYLENE

SEC-EUTYLEENZENE

STYRENE

TERT-QUTYL BENZENE

TETRACHLOROE THEME

TOLUENE

TPH

TPHH

TPHL

TRANS-1,2-OICHLOROETHENE

TRAGS-1,3-DICHLOROPROPENE

TRICHLOROE THENE

VIUYL ACETATE

XYLENES (total)
$10 / 11 / 91$

10.00

Ma

MA

$M$

10.00

10.00

5.00

MA

MA

MA

Wh

MA

MA

s.ou

MA

5.0

5.00

MA

MA

MA

MA

5.00

5.0

MA

s.0 


\section{C-51}

KANSAS CITY : SELECTED ANALYSIS FROA KC99-164.L

Constituent

us/L

\begin{tabular}{|c|c|}
\hline 1,1,9-TRICHLOROE THAME & $5.0 \mathrm{~J}$ \\
\hline $1,1,2,2 \cdot$ IETKACHLOROE THANE & 5.00 \\
\hline 1,1,2-TRICHLOROETKAHE & $5.0 \mathrm{~d}$ \\
\hline 1,1,2-TR!CHLOROTR]FLLUROETH & $5.0 \mathrm{~d}$ \\
\hline 1,1-DICHLOROETHAKE & 5.00 \\
\hline 1,1-DICHLOROETHENE & $5.0 \mathrm{~s}$ \\
\hline 1,2,4-TRICHLOROBERZENE & kA \\
\hline 1,2-DICHLOROBEKZENE & 5.00 \\
\hline 1,2.DICHLOROET TAAEE & 5.00 \\
\hline 1,2-DICHLOROETHENE (totol) & 5.00 \\
\hline 1,2-DICHLOROPROFAKE & $5.0 \mathrm{~s}$ \\
\hline 1,3-DICHLOROBEKIZENE & 5.0 .5 \\
\hline 9,4-DICHLOROEEKIZENE & 5.00 \\
\hline 2-BUTANOKE & $5.0 \mathrm{~s}$ \\
\hline 2- HEXANONE & 5.00 \\
\hline 4-METHYL-2-PERTAKONE & 5.0 \\
\hline RCETONE & 10.00 \\
\hline BENZENE & 5.00 \\
\hline GENZIDIKE & MA \\
\hline EROMOFORM & 5.00 \\
\hline CARBON DISULFIDE & S.0u \\
\hline CARBOW TETRACHLORIDE & $5.0 \mathrm{U}$ \\
\hline CHLOROBEK'ZENE & 5.00 \\
\hline CHLORDOI GROMOMET KANE & 5.0 \\
\hline CHLOROETHANE & 10.00 \\
\hline CHLOROETHEKE & $10.0 \mathrm{U}$ \\
\hline CHLOROFORK & 5.0 \\
\hline CIS-1,3-DICHLOROPROPERE & 5.00 \\
\hline DICHLOROSROMOMET HAHE & $5.0 \mathrm{~s}$ \\
\hline ETHYLEEKZENE & 5.00 \\
\hline FLUOROTRICHL OROMETHAHE & $10.0 \mathrm{~s}$ \\
\hline HEXACHL OROBUTADIENE & HA \\
\hline HETHYL GROMIDE & 10.05 \\
\hline METHYL CHLORIDE & $10.0 \mathrm{~s}$ \\
\hline MEYMYLENE CHLORIDE & 5.01 \\
\hline NAPHTHALENE & in \\
\hline STYRENE & $5.0 \mathrm{U}$ \\
\hline TETRACHLOROETHENE & 5.00 \\
\hline TOLUENE & 5.00 \\
\hline TPHH & NiA \\
\hline TPHL & $\mathrm{MA}$. \\
\hline IRANS- 1,3 -DICHLOROPROPENE & $5 . \infty$ \\
\hline IRICHLOROETHENE & 5.00 \\
\hline CYLERES (rotal) & 5.00 \\
\hline
\end{tabular}

$01 / 10 / 92$

as

. a

.

. $\infty$

.0.

as

5.0

.00

or

$\infty$

.0u

.

. .

5.00

10.01

$0.0 \mathrm{~s}$

5.00

5.05

5.00

5.00
Somple Date 


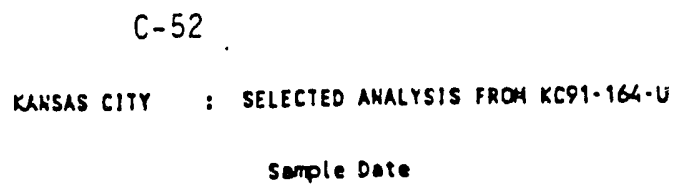

Constituent

ug/L

$01 / 10 / 92$

\begin{tabular}{|c|c|}
\hline $9,9,9 \cdot$ TEICKLOROETHAHE & 5.00 \\
\hline $1,1,2,2-1 E T R A C H L$ OROE THAME & 5.0 \\
\hline $1,1,2$-IRICHLOROETHAHE & 5.00 \\
\hline $1,1,2$ TRICHLOROTRIFLUOROETH & 5.00 \\
\hline 1,1-DICHLOROETHANE & 5.00 \\
\hline 1, 1-DICHLOROETHEHE & 5.00 \\
\hline $1,2,6$-TRICHLOROSENIZENE & MA \\
\hline 1.2-DIEHLOROSEKZENE & 5.00 \\
\hline 1,2-0ICKL OROETHAHE & 5.00 \\
\hline 9,2-DICHLOROETHEKE (total) & 5.00 \\
\hline 1,2-OICHLOROPROPAHE & $5 . \alpha$ \\
\hline 1,3-DICHLOROEEK'ZENE & s.ov \\
\hline 1,4-DICHLOROSEKIZEHE & 5.00 \\
\hline 2-BUTANONE & 5.0 \\
\hline 2-HEXANONE & 5.00 \\
\hline 4- METHYL-2-DEHTANOWE & 5.00 \\
\hline ACETOKE & 10.00 \\
\hline BENZENE & $5 . \alpha$ \\
\hline BENZ:OINE & NA \\
\hline BROMOF ORH & 5.00 \\
\hline CARBOW DISULFIDE & 5.00 \\
\hline CARBON IETRRCHLORIDE & 5.00 \\
\hline CHLOROEEHZENE & 5.00 \\
\hline CHLORDOI EROMOMETHANE & 5.00 \\
\hline CHLOROETHANE & 10.00 \\
\hline CHLOROETHEKE & 10.00 \\
\hline CHLOROFORH & 5.00 \\
\hline CIS-1,3-0ICHLOROPROPENE & 5.00 \\
\hline DICHLLROSROMOMETHANE & 5.00 \\
\hline ETHYLSENZENE & 5.00 \\
\hline FLUOROTRICHLOROMET THANE & 10.00 \\
\hline HEXACHLOROSUTADIENE & wis \\
\hline METKYL BROMIDE & 10.00 \\
\hline METHYL CHLORJOE & 90.00 \\
\hline METHYLENE CHLORIOE & 5.00 \\
\hline NAPHTHALENE & NA \\
\hline STYRENE & 5.00 \\
\hline TETEACHLOROETHENE & 5.0u \\
\hline TOLUENE & 5.00 \\
\hline IPHK & NA \\
\hline TPHL & NA \\
\hline TRANS-1,3-DICMLOROPROPENE & 5.00 \\
\hline TRICHLOROETHENE & $5.0 \mathrm{~s}$ \\
\hline XYLENES (tOTAl) & 5.00 \\
\hline
\end{tabular}




\section{C -53}

KANSAS CIIY : SELECTED AKALYSIS IROM KCO9-164-L

Const ituent

Sumple date

ug/L

$04 / 27 / 92$

\begin{tabular}{|c|c|}
\hline $1,1,9$-TRICHLOROETHANE & $5.0 \mathrm{U}$ \\
\hline 1,1,2,2-TETRACHL OROET THAME & 5.00 \\
\hline 1,1,2-TRICHLOROETHAKE & $5.0 \mathrm{~J}$ \\
\hline 1,1,2-TRICKLOROTRIFLLOROEIH & 5.00 \\
\hline 1,1-DICHLOROETHRHE & 5.00 \\
\hline 1,9-01CHLORO 1 HENE & 5.00 \\
\hline 1,2-DICHLOROSEKRENE & 5.00 \\
\hline 1,2-DICHLOROETHAHE & 5.04 \\
\hline 1,2-DICHLOROE T HENE (TOTOL) & 32.0 \\
\hline 1,2-DICHLOROPROPANE & 5.00 \\
\hline 1,3-DICHLOROSELIZENE & 5.00 \\
\hline 1,4-DICHLORODERLERE & 5.00 \\
\hline 2-BUTANOWE & 5.04 \\
\hline 2- HEXANOWE & 5.00 \\
\hline 4-METKYL-2-PERTAHOHE & $5.0 \mathrm{U}$ \\
\hline ACETONE & $10.0 \mathrm{U}$ \\
\hline BENZENE & 5.00 \\
\hline BROMOFORM & s.ou \\
\hline CARBOW DISULFIDE & 5.00 \\
\hline CARBOW TETRACHLORIDE & 5.00 \\
\hline CHLOROBENZENE & 5.04 \\
\hline CHLORDOI IBROMOMETHAKE & $5.0 \mathrm{U}$ \\
\hline CHLOROETHAKE & $10.0 \mathrm{~d}$ \\
\hline CHLOROETHEME & 10.00 \\
\hline CHLOROFORM & 5.00 \\
\hline CI5-1,3-DICHLOROPROPENE & 5.00 \\
\hline DICHLOROBROMOME THANE & 5.00 \\
\hline ETHYLEENZENE & 5.00 \\
\hline FLUOROTRI CHL OROME THANE & 10.00 \\
\hline METHYL GROKIDE & $10.0 \mathrm{~s}$ \\
\hline METHYL CHLORIDE & 10.00 \\
\hline METHYLENE CHLORIDE & 5.00 \\
\hline TYRENE & 5.00 \\
\hline IETRACHLOROE THEME & 5.00 \\
\hline OLUENE & 5.00 \\
\hline PHH & NA \\
\hline 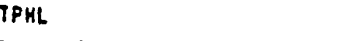 & NA \\
\hline KANS-1,3-DICHLORO & 5.00 \\
\hline RICHL ORUE T HENE & 5.00 \\
\hline YLENES (total) & 5.00 \\
\hline
\end{tabular}




\section{C -54}

KANSAS CITY : SELECTED AKALYSIS FROA KCS1.164.U

Sample Date

Constituent

$04 / 27 / 92$

\begin{tabular}{|c|c|}
\hline 1,1,9-TRIEHLOROET HANE & $5 . \infty$ \\
\hline $1,1,2,2$ - TETHACKLOROET TANE & $5.0 \mathrm{~d}$ \\
\hline 1,1,2-TRICHLOROETHANE & $5 . \infty$ \\
\hline 1,1,2-TRICHLOROTRIFLUOROETH & 5.00 \\
\hline 1,1-DICHL OROET HAHE & 5.00 \\
\hline 1,1-DICHLOROET TE HE & 5.0u \\
\hline 1,2-DICHLOROSEKZENE & 5.00 \\
\hline 1,2-DICHLDROETHAME & 5.00 \\
\hline 1,2-DICHLOROETKENE (TOLOI) & 9.0 \\
\hline 1,2-DICHLOROPROFAKE & 5.00 \\
\hline 1,3-OICHLOROBENZEENE & 5.01 \\
\hline 1,4-DICHLORDSENZENE & 5.00 \\
\hline 2-BUTAMOAE & 5.00 \\
\hline $2 \cdot$ HEXANOWE & 5.0 \\
\hline 4-METHYL-2-PENTAMONE & 5.00 \\
\hline ACETONE & 10.00 \\
\hline BENZENE & 5.00 \\
\hline SROWOFORM & 5.00 \\
\hline CARBOW DISULFIDE & 5.00 \\
\hline CARBON TETRACMLORIDE & 5.00 \\
\hline CHLOROGENZEME & 5.00 \\
\hline CHLORDO I BROMOME T KAKE & $5.0 \mathrm{U}$ \\
\hline CKLOROET HAME & $10.0 \mathrm{~s}$ \\
\hline CKLOROET HENE & 10.0 \\
\hline CHLOROFOEK & 5.0 \\
\hline CI5-1,3-DICHLOROPROPENE & 5.00 \\
\hline DICHLOROSROMOMETHKHE & 5.0 \\
\hline ETKYLBEKZENE & 5.00 \\
\hline FLLOROTR I CKL OROME T HANE & 10.00 \\
\hline METHYL ERONIDE & 10.00 \\
\hline METHYL CHLORIDE & 10.00 \\
\hline METHYLENE CHLORIDE & 5.00 \\
\hline STYRENE & 5.00 \\
\hline TETRUCKLOROETHEHE & 5.00 \\
\hline TOLUEKE & 5.04 \\
\hline TPHH & in \\
\hline TPHL & in \\
\hline TRAMS-1,3-01CHLOROPROPENE & 5.00 \\
\hline TRICHLOROETHEME & 5.05 \\
\hline XYLEKES (roial) & s.ou \\
\hline
\end{tabular}


KANSAS CITY : SELECTED AMALYSIS FROA KC91-165-L

Constituent

$u g / 6$

$10 / 91 / 91$

$1,1,1,2$ - TETRACHLOROE THANE

$1,1,1$ - IRICHLOROETHANE

$1,1,2,2$ - TETRACHLOROE THANE

$1,1,2$ - TRICHLOROETHANE

$1,1,2$ - TRICHLOROTRIFLUOROETH

1,1 -DICHLOROE THANE

1,1 -DICHLOROETHENE

1,1-DICHLOROPROPENE

$1,2,3$-TRICHLOROBENZENE

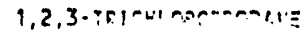

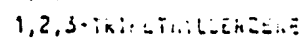

$1,2,6$-TRICHLOROBENZENE

1,2-01BROMO-3-CKLOROPROPANE

$1,2 \cdot 01$ BRomoet thaNe

1,2-0ICHLOROEENZENE

1,2-DICKLOROETHANE

1,2-DICKLOROETHENE (total)

1,2-DICHLOROPROPANE

1,3,5-TRIKET THYBENZENE

3-DICHLOROBEMZENE

1,3-DICHLOROPROPANE

1,6-OICHLOROBEKZENE

2,2-DICHLOROPROFANE

2-BUTANONE

2-CHLOROETHYLVIUYL ETHER

2-CHLOROTOLUENE

2- HEXUNONE

4-CHLOROTOLUEME

4-METKYL-2-PENTAMONE

ACETONE

ACROLEIN

ACRYLOWITRILE

BEMZEME

BROMOBENZENE

BROMOCHL OROME THANE

BROMOFORH

CAREOW DISULFIDE

CARBOW TETRACMLORIDE

SHLOROBENZEHE

CHLORDOI I BROMOMETHAME

CHLOROETHANE

CHLOROETHEME

CHLOROFORM

CIS-1,2-DICKL OROETHENE

-15-1.3-01CHLOROPROPENE

I bromomethane

DICHLOR OBROMOME TKANE

DICHLORDO I F LUOROME TKANE

ETHYLBENZEME
MA

5.00

s.o

5.00

5.00

5.01

5.00

Ma

MA

in

i.n

MA

MA

MA

s.ou

5.01

160.0

5.00

HA

5.00

NA

5.0u

MA

S.OU

MA

ua

5.0

NA

5.00

is..?

MA

HA

5.00

NA

NA

5.0u

5.00

5. 0

5. 0

5.00

10.00

29.0

5. 00

kA

5.00

uA

5.00

NA

5.00

Semple Dote 
KANSAS CITY : SELECTED AKALYSIS FROM KC91.165.L

Constituent

ug/t

PLLOROTRICHLOROMETHAKE

HEXUCHL OROBUT NO IEME

ISOPROPYLBENZENE

M-XYLENE

METHYL BROMIDE

METKYL CHLORIDE

METHYLEME CKLORIDE

M-BUTYLBENZENE

W.PROPYLBENZENE

MAPHTHALENE

O.XYLENE

P. ISOPROPYLTOLUENE

P.XYLENE

SEC-BUTYLBENZENE

STYREME

TERT-BUTYLBEKZEME

TETRACHLOROETHENE

TOLUENE

TPH

TPHM

TPHL

TRANS-1, 2-DICHLOROETHENE

TRANS-1,3-DICHLORCPROPENE

TRICHLOROETHENE

VIUYL ACETATE

XYLEMES (TOTSI)
10.00

MA

WA

Ma

10.00

10.00

5. 0 s

MA

MA

WA

NA

MA

NA

WA

5.00

MA

s. $\infty$

s.0

ua

M

m

MA

5.00

160.0

un

5.00
Somple Date

$111 / 91$ 


\section{C -57}

KANSAS CITY : SELECTED AMALYSIS FROM KC91-165.U

Constituent

us/l

$1,1,1,2$ - TE TRACMLOROE THAHE

$1,1,1$ - TRICHLOROC:THANE

$1,1,2,2$ - TETRACHLOROETHANE

$1,1,2$ - TRICHLOROETHANE

1,1,2-TRICHLOROTR!FLUOROETH

1,1-DICHLOROE THANE

1,1-OICHLOROETHENE

1,1.DICHLOROPROPEME

$1,2,3$ - TRI CKL OROSE NZENE

$1,2,3$-TRICHLOROPROPANE

$1,2,3$-TRIMETHYLBENZENE

$1,2,4-$ TRICHLOROBENZENE

1,2-DICHLOROEET2ENE

1,2-DICHLOROETHANE

1,2-DICHLOROETHENE (TOTAl)

1,2-DICKLOROPROPANE

1,3,5- TRIMETHYL BEMZENE

3-01CHLOROSENZENE

1,3-DICHLOROPROPANE

1,4-DICHLOROBENZEME

2,2-DICHLOROPROPANE

2- BUTANONE

2-CHLOROETHYLVIHYL ETHER

2- CHLOROTOLUENE

2- HEXANOWE

4- CHLORDIOLUENE

4-METHYL-2-PENTANONE

ACETONE

ACROLEIN

ACRYLOWITRILE

BEMZENE

BromosenzENE

BROMOCHL OROMET KANE

BRONOFORM

CARBOW DISULFIOE

CARBOW TETRACHLORIDE

CHLOROBENZENE

CHLORDO IBROMOMET THANE

CHLOROE THANE

CHLOROETHENE

CKLOROFORM

CIS-1,2-DICHLOROET HEME

C15-1,3-01CKLOROPROPENE

IBROMONE TKAHE

DICHL OROBROMOME THANE

DICHLORDO I FLLOROME T HAHE

ETMYLBEHZENE
Ma

s.ou

5.01

5.0

5.0

5.00

5.00

MA

KA

XA

MA

wA

s.w

5.0

8.0

5.00

MA

S.QU

WA

5.00

MA

5.00

MA

MA

5.0

MA

5.00

10.00

WA

MA

s.ou

na

M

5.00

5.00

5.00

5.00

5.00

10.00

10.00

s.0u

NA

5.00

MA

S.00

MA

5.00

Semple Dote 
KANSAS CITY : SELECTED AKALYSIS EROM KC91-165-U

Constituent

$u / l$

FLUOROTRICHL OROMETHANE

HEXACHLOROSUTADIENE

ISOPROPYLBEHZEME

X-XYLEAE

METHYL BROMIDE

METKYL CHLOR IDE

METHYLEME CKLORIDE

M-BUTYLBENIEKE

M-PROPYLBENZEME

MAPHTHALENE

O-XYLENE

P.1SOPKOPYLTOLUENE

P-XYLENE

SEC-RUTYLBEKZENE

STYRENE

TERT-BUTYLBENZENE

TETRACHLOROE THE YE

TOLUEKE

TPH

itK:?

TPKL

TRANS-1,2-DICHLOROETHENE

TRANS-1,3-DICHLOROMOPENE

TRICHLRRDETHEME

VIMYL ACETATE

XYLENES (total) 10.0u

MA

ma

ma

10.w

10.00

5.00

Ma

MA

MA

MA

KA

MP.

NA

5.0u

ka

5.00

5.00

wA

MA

$m$

HA

s.os

5. 01

MA

5.00

Somple Date 
RANSAS CITY : SELECTED ANALYSIS FROM KC91.165.L

Sumple Date

Constituent

ug/L

$01 / 10 / 92$

\begin{tabular}{|c|c|}
\hline 1,1,9-TRICHLOROETHANE & 5.00 \\
\hline 1,1,2,2-TETKACHLOROETHAHE & 5.00 \\
\hline 1,9,2-TRICHL OROETHAME & $5.0 \mathrm{v}$ \\
\hline 1,1,2-TRICKLOROTR!FLUOROETK & 5.00 \\
\hline 9,9-DICHLOFOETHALE & 6.0 \\
\hline 9.9-DICHLOROETHENE & $5.0 \mathrm{~s}$ \\
\hline 1,2,4-TRJCHLOROBERIZEHE & ma \\
\hline 1,2-DICHLOROSEK'ZERE & 5.00 \\
\hline 9,2-DSCHLOADETHAKE & $5.0 \mathrm{~s}$ \\
\hline 1,2-DICHLOROETHEHE (YOIBI) & 400.0 \\
\hline 1,2-DICHLOEOFROPRHE & 5.00 \\
\hline 1,3-DICHLOROSERZENE & 5.00 \\
\hline 1,4-DJCKLORCSENZENE & 5.00 \\
\hline 2-BUTANONE & 5.00 \\
\hline 2-HEXANONE & 5.00 \\
\hline 4-METHYL-2-PERTARONE & $5.0 \mathrm{~d}$ \\
\hline ACETOHE & 10.00 \\
\hline BENZENE & 5.00 \\
\hline BEH2:01KE & NA \\
\hline BROMOFORM & $5.0 \mathrm{~d}$ \\
\hline CARBOW DISULFIDE & 3.00 \\
\hline CAREON TETEACKLORIDE & 5.00 \\
\hline CHLOROBEKIEEKE & 5.01 \\
\hline CHLORDO I EROMDMET THAKE & $5.0 \mathrm{~s}$ \\
\hline CHLOROETHANE & 10.00 \\
\hline CHLOROETHEAE & 10.00 \\
\hline CHLOROFOEM & $5.0 \mathrm{~s}$ \\
\hline CIS-9,3-DICHLOROPROPENE & 5.00 \\
\hline DICHL OROEROMIME THANE & 5.00 \\
\hline ETHYLBELIZENE & 5.00 \\
\hline FLLOROTRICHLOROMETHANE & $10.0 \mathrm{U}$ \\
\hline HEXACHLOROBUTADIENE & NA \\
\hline METHYL SRONIDE & 10.00 \\
\hline METHYL CHLORIDE & 100.0 \\
\hline METHYLENE CHLORIDE & $5 . \infty$ \\
\hline NAPHTHALENE & WA \\
\hline STYRENE & 5.00 \\
\hline TETRACHLOROETHENE & 5.00 \\
\hline TOLUENE & 5.00 \\
\hline IPHH & Win \\
\hline TPHL & HA \\
\hline TRANS-1,3-DICHLOROPROPENE & 5.00 \\
\hline YRICKL OROETKENE & 260.0 \\
\hline XYLENES (TOTAl) & 5.0 \\
\hline
\end{tabular}




$$
\text { C- } 60 \text {. }
$$

KAKSAS CITY : SELECTED AHALYSIS FROA KC99-165.U

Constituent

UE/L

\begin{tabular}{|c|c|}
\hline $1,1,1 \cdot$ TRICHLOROETHAME & 5.00 \\
\hline $1,1,2,2-1 E T R A C H L$ OROE THAHE & $5.0 \mathrm{~d}$ \\
\hline $1,1,2 \cdot$ TRICHLOROETHAME & 5.00 \\
\hline $1,1,2$-TRIEKL OROTE] ILLOROETH & 5.00 \\
\hline 1,1-DICHL OAOETHLHE & 5.00 \\
\hline 1,9.DICHLOROETHEHE & 5.01 \\
\hline $1,2,4$-TEJCHLOROSERIEENE & MA \\
\hline 1,2-DICHLOROEENLENE & 5.00 \\
\hline 1,2-DICHL OROET THANE & 5.w \\
\hline 1,2-DICKLOROETHEHE (TOTAl) & 7.0 \\
\hline 1,2-DICHLOROPROFRHE & 5.00 \\
\hline 1,3-DICHLOROEERZZENE & 5.0u \\
\hline 1,6-DICHLOROEEATELAE & 5.00 \\
\hline 2-BUTANONE & 5.0u \\
\hline 2- HEXSHONE & 5.01 \\
\hline 4-METKYL-Zे-PEKTANONE & 5.00 \\
\hline ACETONE & 10.00 \\
\hline SERZENE & 5.00 \\
\hline GELZIDINE & NA \\
\hline BROMOFORM & s.ou \\
\hline CARBOW DISULFIDE & 5.00 \\
\hline CAREOW TETRACHLORJDE & 5.00 \\
\hline CHLOROEERIZENE & 5.00 \\
\hline CHLORDO I BROMOMET HANE & 5.00 \\
\hline CHLOROE T HAKE & 10.00 \\
\hline CHL OROE THENE & 10.00 \\
\hline CHLOROFORM & s.0u \\
\hline C15-9,3-DICHLOROPROPENE & 5.00 \\
\hline DICHL OROER RMOMET TKAME & $5.0 \mathrm{~J}$ \\
\hline ETHYLEEAIZENE & 5.00 \\
\hline FL UOROTR! CHLOROME THAME & 10.00 \\
\hline HEXACHL OROBUTRDIENE & Na \\
\hline METHYL BROMIDE & 10.00 \\
\hline METHYL CHLORIDE & $10.0 \mathrm{~s}$ \\
\hline METHYLENE CHLORIDE & 5.0 \\
\hline NAPHTHALENE & wa \\
\hline STYKEKE & 5.01 \\
\hline TETRKCHLOROEIHEHE & $5.0 \mathrm{~d}$ \\
\hline TOLUENE & $5.0 \mathrm{~J}$ \\
\hline IPHK & in \\
\hline IPHL & WA \\
\hline RANS- 9,3 -DICHLOROPROPENE & 5.00 \\
\hline IEICHL OROETHELE & 5.00 \\
\hline CYLEKES (TOR $/$ l) & $5 . a$ \\
\hline
\end{tabular}

$01 / 10 / 92$

ou

.ov

7.0

5.00

s.

5.00

5.00

5.00

5.00

5.00

5.0

0.00

5.00

5.00

5.00

0.00

5.00

s.ou

5.00

in

5.00

5.00
Sample Date 
KANSAS CIIY : SELECTED AMALYSIS FROM KC99-165.L

\section{Constituent}

us $/ L$

$1,1,1$ - TRICHLOROETKAKE

$1,1,2,2-$ TETKACHLOROETHANE

$1,1,2$-TRICHLOROETKANE

$1,1,2$-TRICHLOROTKIFLLOROETH

1,1-DICHLOROE THANE

1,1-DICHLOROETHENE

1,2-DICHLOROEEKIZENE

1,2-DICHLOROETHANE

$1,2 \cdot$ DICHLOROETHENE (TOTA1)

1,2-DICHLOROPROPAHE

1,3-DICHLOROEENZEHE

1,4-DICHLOROSENZENE

2- BUTAHONE

2- HEXANONE

4-METHYL-2-PENTAHONE

ACETONE

BENZEHE

GROMOF ORM

CARBON DISULFIDE

CARBON TETRACHLORIDE

CHLOROBENZENE

CHLORDOI IBROMOMET THAKE

CHLOROETHANE

CHLOROETHENE

CHLOROFORY

CIS-1,3-DICHLOROPROPENE

DICHLOROBROMOME THANE

ETHYLBENZENE

FLUOROTRI CHLOROHET HANE

METHYL BROMIDE

METHYL CHLORIDE

RETHYLENE CHLORIDE

STYRENE

TETRACHLOROETHENE

TOLUENE

TPHH

TPHL

TRANS-1,3-DICHLOROPROPENE

TRICHLOROET MENE

XYLENES (iCial)
5.00

5.00

5.00

5.00

5.00

8.0

5.00

5.00

890.0

5.00

5.00

5.00

5.00

5.00

5.00

10.00

5.00

5.00

5.00

5.00

5.00

5.01

10.00

180.0

5.00

5.00

5.00

5.00

10.00

10.00

10.00

5.00

5.00

5.00

5.00

MA

HA

5.00

1300.0

5.00

Sumple Date 
KANSAS CITY : SELECTED AKALYSIS TROM KCOM-1E5.U

Constituent

vell

$1,1,9$ - TRICHLOROETKANE

$1,1,2,2$-TETRACHLOROET TANE

$1,1,2$ - TRICHLOROEI TAME

$1,1,2$-TRICHLOROTRIFLUOROETH

1,9-DICHLOROE I HALE

1,9-DICHLOROETHENE

1,2-DICHLOROSENZENE

$1,2-01$ CHLOROET KAME

1,2-01CHLOROETHENE (toTal)

1,2 -DICHLOROPROPANE

1,3-01CHLOP.05EK2ER'E

1,4-DICHLORCEEKZEKE

2- BUTANOHE

2- KEXAHONE

4-METHYL-2-PELTAHONE

ACETOWE

BENZENE

BROMOF ORM

CARBOW DISULFIDE

CARBOW TETRACHLORIDE

CHLOROBEK2EME

CHLORCOI IR OMOMET THANE

CHLOROET TAME

CHLOROETHENE

CHLOROFORK

CIS-1,3-DICHL DROPROPENE

DICHL OROSROMOUE THAME

ETHYLBENZENE

FLUOROTRICHLOROMETHANE

METHYL BROMIDE

METHYL CHLORIDE

METUYLLENE CHLORIDE

STYRENE

TETRACHLOROETHEME

TOLUEME

TPHH

IPHL

TEANS-1,3-DICHLOROPROPENE

TRICHLOROE T WE ME

XYLENES (TOT:I)
5.00

5.00

5.00

5.01

5.00

5.00

s.ov

5.0u

5.00

s.os

$5.0 \mathrm{~J}$

5.01

5.00

5.00

5.00

10.00

5.00

s.o

5.00

$5.0 \mathrm{~s}$

5.00

5.00

10.00

$10.0 \mathrm{~s}$

5.00

5.00

5. 01

5.00

10.00

$10.0 \mathrm{~s}$

10.01

5.00

5.00

5.00

s.o

MA

ind

5.00

$5.0 \mathrm{~J}$

5.00

Supple Dete 
ORNL/TM-12226

\section{INTERNAL DISTRIBUTION}

$\begin{aligned} \text { 1. } & \text { B. A. Berven } \\ 2 . & \text { J. R. Davidson } \\ 3 . & \text { K. S. Dickerson } \\ 4 . & \text { F. G. Gardner } \\ \text { 5. } & \text { D. W. Greene } \\ 6 . & \text { S. C. Hall } \\ 7 . & \text { M. K. Jensen } \\ 8 . & \text { N. E. Korte } \\ 9 . & \text { A. D. Laase } \\ 10-15 . & \text { C. A. Little } \\ 16 . & \text { C. A. Muhr }\end{aligned}$

1. B. A. Berven

2. J. R. Davidson

3. K. S. Dickerson

4. F. G, Gardner

5. D. W. Greene

6. S. C. Hall

7. M. K. Jensen

8. N. E. Korte

6. C. A. Muhr

\section{EXTERNAL DISTRIBUTION}

31. J. L. Baker, Allied Signal, Inc., Kansas City Division, 2000 E. 95th Street, Kansas City, Missouri 64131

32. Rex Buchannan, Kansas Geological Survey, West Campus, University of Kansas, Lawrence, Kansas 66047

33. D. S. List, Oak Ridge Institute of Science and Education, P. O. Box 2567, Grand Junction, Colorado 81502

34. D. A. Locke, Oak Ridge Institute of Science and Education, P. O. Box 2567, Grand Junction, Colorado 81502

35. D. C. Marty, Oak Ridge Institute of Science and Education, P. O. Box 2567, Grand Junction, Colorado 81502

36. M. B. Mumby, Oak Ridge Institute of Science and Education, P. O. Box 2567, Grand Junction, Colorado 81502

37. R. M. Schlosser, Oak Ridge Institute of Science and Education, P. O. Box 2567, Grand Junction, Colorado 81502

38. S. M. Smith, Oak Ridge Institute of Science and Education, P. O. Box 2567, Grand Junction, Colorado 81502

39. G. K. Stowe, Oak Ridge Institute of Science and Education, P. O. Box 2567, Grand Junction, Colorado 81502

40. J. L. Zutman, Oak Ridge Institute of Science and Education, P. O. Box 2567, Grand Junction, Colorado 81502

41. Office of Assistant Manager, Energy Research and Development, Oak Ridge Operations Office, P. O. Box 2001, Oak Ridge, Tennessee 37831-8600

42 - 51. Office of Scientific and Technical Information, U.S. Department of Energy, P. O. Box 62, Oak Ridge, Tennessee 37831 

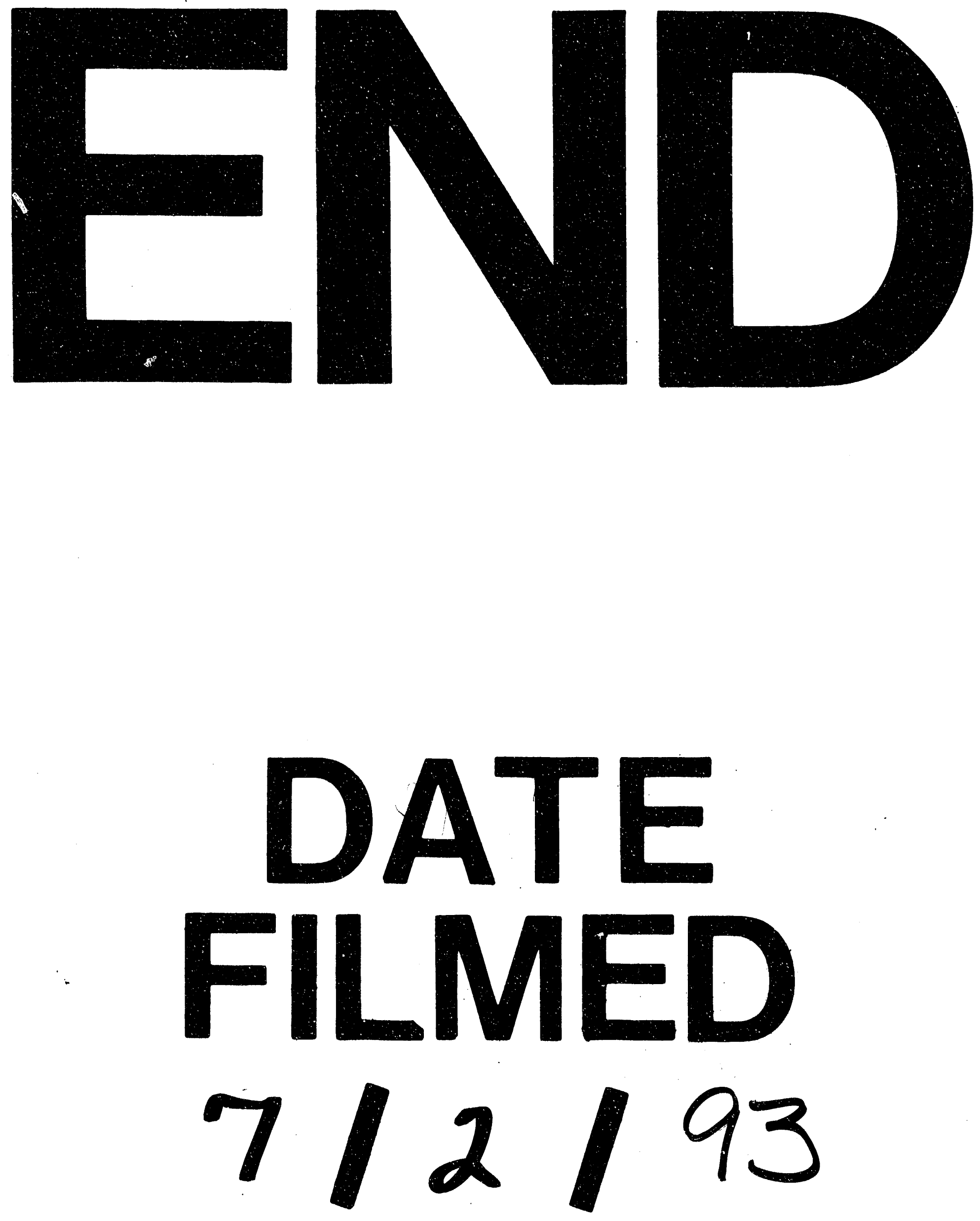
